\title{
OPEN Evaluation of indigenous aromatic rice cultivars from sub-Himalayan Terai region of India for nutritional attributes and blast resistance
}

\author{
Debayan Mondal ${ }^{1}$, Prudveesh Kantamraju ${ }^{1}$, Susmita $\mathrm{Jha}^{2}$, Gadge Sushant Sundarrao ${ }^{3}$, \\ Arpan Bhowmik ${ }^{4}$, Hillol Chakdar ${ }^{5}$, Somnath Mandal ${ }^{1}$, Nandita Sahana ${ }^{1 凶}$, Bidhan Roy ${ }^{3}$, \\ Prateek Madhab Bhattacharya ${ }^{2}$, Apurba Kr Chowdhury ${ }^{2}$ \& Ashok Choudhury ${ }^{6}$
}

Indigenous folk rice cultivars often possess remarkable but unrevealed potential in terms of nutritional attributes and biotic stress tolerance. The unique cooking qualities and blissful aroma of many of these landraces make it an attractive low-cost alternative to high priced Basmati rice. Sub-Himalayan Terai region is bestowed with great agrobiodiversity in traditional heirloom rice cultivars. In the present study, ninety-nine folk rice cultivars from these regions were collected, purified and characterized for morphological and yield traits. Based on traditional importance and presence of aroma, thirtyfive genotypes were selected and analyzed for genetic diversity using micro-satellite marker system. The genotypes were found to be genetically distinct and of high nutritive value. The resistant starch content, amylose content, glycemic index and antioxidant potential of these genotypes represented wide variability and 'Kataribhog', 'Sadanunia', 'Chakhao' etc. were identified as promising genotypes in terms of different nutritional attributes. These cultivars were screened further for resistance against blast disease in field trials and cultivars like 'Sadanunia', 'T4M-3-5', 'Chakhao Sampark' were found to be highly resistant to the blast disease whereas 'Kalonunia', 'Gobindabhog', 'Konkanijoha' were found to be highly susceptible. Principal Component analysis divided the genotypes in distinct groups for nutritional potential and blast tolerance. The resistant and susceptible genotypes were screened for the presence of the blast resistant pi genes and association analysis was performed with disease tolerance. Finally, a logistic model based on phenotypic traits for prediction of the blast susceptibility of the genotypes is proposed with more than $80 \%$ accuracy.

Rice (Oryza sativa L.) is one of the most popular staple foods consumed by more than half of the world's population including Asians. Along with many south Asian countries, rice is widely consumed in India as cooked, puffed and pounded forms. Aromatic rice pertaining to a special group of rice is regarded highly due to presence of excellent aroma and superior grain quality. The Indian subcontinent is blessed with nature's gift of Basmati rice popular among consumers as superior, scented, long slender grain rice and fetches premium price in the national and international markets. Along with the popular Basmati rice genotypes a hand full of traditional heirloom rice genotypes also possess excellent aroma, making many of these unrecognized landraces as an attractive low-cost alternative to high priced Basmati rice. West Bengal takes pioneer position in rice production in India and has considerably large diversity in cultivated folk rice genotypes. Two such cultivars of West Bengal, 'Tulaipanji' and 'Gobindabhog', have already been GI tagged and gained considerable attention in the international market for it's uses in multinational cuisines and were featured in global sporting events ${ }^{1}$. Sub-Himalayan Terai region of West Bengal harbors considerably wide variability in rice cultivation and is identified as a hotspot of growing nonBasmati aromatic rice ${ }^{2}$. The rich agrarian heritage of the ancient tribe residents of this region, the 'Rajbonshis'

\footnotetext{
${ }^{1}$ Department of Biochemistry, Uttar Banga Krishi Viswavidyalaya, Pundibari, Coochbehar 736165, India. 'Department of Plant Pathology, Uttar Banga Krishi Viswavidyalaya, Pundibari, Coochbehar 736165, India. 'Department of Seed Science and Technology, Uttar Banga Krishi Viswavidyalaya, Pundibari, Coochbehar 736165, India. ${ }^{4}$ ICAR-Indian Agricultural Statistics Research Institute, Pusa, New Delhi 110012, India. ${ }^{5}$ ICAR-National Bureau of Agriculturally Important Microorganisms, Mau Nath Bhanjan, UP 275103, India. ${ }^{6}$ Soil Microbiology Laboratory, Regional Research Station, Uttar Banga Krishi Viswavidyalaya, Pundibari, Coochbehar 736165, India. ${ }^{\boxplus}$ email: nanditasahana@gmail.com
} 
has predominant role in conserving the rice agrobiodiversity of this region. The abundance of monsoon and large number of rivers in this region has facilitated the formation of Buri Balason rice bowl, Teesta-Dharala rice bowl, Kranti-Golabari rice bowl, Mekhliganj rice bowl etc. where aromatic landraces of rice, locally known as 'bhogdhan' are cultivated since time immemorial ${ }^{3,4}$. Besides aroma these local indigenous rice landraces, collectively regarded as the folk rice cultivars, offers genetic and nutritional diversity, sustainability, reduction in the carbon footprints and imports, tolerance to many diseases and pests due to the broadening of the gene pool, and adaptability to the local soil and climatic conditions ${ }^{5}$. The introduction of High-Yielding Varieties (HYV) in rice cultivation has led to gradual disappearance of folk rice cultivars from farmers field since green revolution ${ }^{6-8}$ which has resulted in survival of only a handful of these genotypes ${ }^{9-13}$. Although the replacement of these aromatic landraces with high- yielding modern varieties ensures good yield but enforces colossal threat to the rice agrobiodiversity resulting permanent damage to the rice ecosystem of different states of India ${ }^{14}$.

Aromatic landraces have comparatively low yield potentiality than HYVs, but offer considerable variation in agronomic, phenotypic, nutritional and disease tolerance traits. Inspite of their low yield potential, rice landraces have been proven valuable for resistance to rice blast disease in past ${ }^{15,16}$. The sub-Himalayan terai region being very hot and humid in nature, favors the spread of blast disease caused by fungus Magnaporthe oryzae, the most dreaded diseases in all rice growing regions of the world ${ }^{17}$. Profuse leaf blast is very common in this region causing significant yield loss of rice genotypes. Proper screening of the heirloom genotypes for the occurrence and severity of blast disease in this region has not been conducted till date and sources of resistance have not been investigated to explore the gene specificity. Blast pathogen is believed to follow the gene for gene hypothesis ${ }^{17,18}$. More than hundred blast-resistant (R) genes and around 350 QTLs has been reported conferring resistance to blast disease $\mathrm{e}^{15,19}$ among which many has been cloned and biochemically characterized. There are absolutely no studies on the availability of blast-resistant (R) genes in these landraces. Mining of these $\mathrm{R}$ genes in these landraces is the need of the hour to combat region specific blast strain as the co-evolution of these genetically diverse landraces and the blast pathogen from time immemorial has enabled these genotypes with tolerance to the specific strain of the pathogen.

In the present study, we have performed genetic diversity analysis of locally grown scented aromatic landraces and determined the nutritive potential of these heirloom genotypes. The genotypes were also screened for resistance/tolerance to blast disease and suitable aromatic landraces were identified which can be used for future breeding programs. Allele mining for known source of resistance in these cultivars was performed. Our results portray the prospect of these scented landraces in terms of nutritional quality and blast disease resistance for the first time.

\section{Materials and methods}

Plant materials. The indigenous cultivars were collected from different parts of sub-Himalayan region of West Bengal and neighboring states. These genotypes are maintained in Uttar Banga Krishi Viswavidyalaya rice repository after purity breeding. For morphological characterization of the genotypes and screening for blast disease these genotypes were evaluated for two consecutive years (2018 and 2019 kharif season) in the field of Uttar Banga Krishi Viswavidyalaya Instructional Farm. IR64 was used as blast resistant check and 'Gotra bidhan' as local blast susceptible check.

Field practices. The experimental field was divided into seventy-four plots of $5 \mathrm{~m} \times 3 \mathrm{~m}$ size, having $1.5 \mathrm{ft}$ spacing between plots and a $3 \mathrm{ft}$ footway around the field. After seed viability test with $1 \%$ brine water, seed treatment was performed with tricyclazole $(2 \mathrm{~g}$ per $\mathrm{kg}$ of seed) and sown in seedbeds. Twenty-five day old seedlings were transplanted in the main field with plant to plant spacing of $25 \mathrm{~cm}$ and line to line spacing of $50 \mathrm{~cm}$. Fertilizer dose of $\mathrm{N}: \mathrm{P}_{2} \mathrm{O}_{5}: \mathrm{K}_{2} \mathrm{O}_{5}$ at the rate of 70:50:40 kg/hectare (w:w) were applied. Half of ' $\mathrm{N}$ ' has been applied as basal dose. Remaining half of ' $\mathrm{N}$ ' was divided and applied after 30 days after transplanting (DAT) and before flowering stage. During the experiment, the daily weather reports having the parameters like rainfall $(\mathrm{mm})$, maximum and minimum temperature $\left({ }^{\circ} \mathrm{C}\right)$ and maximum and minimum relative humidity percentage were retrieved from the Integrated Agromet Advisory Services (Gramin Krishi Mousam Seva), UBKV, Coochbehar.

Plant phenotypic parameters. All the phenotypic parameters were evaluated in two replications each year. Plant height, tiller number per plant, panicle number per plant, panicle length and leaf angles were measured after the panicle maturation whereas hundred seed weight, filled grain per panicle, grain length, grain width and yield parameters were taken after harvest and drying of the seed material. In each case mean value was calculated for both the years as an average of at least thirty plants taken from two replicative blocks. The upper leaf and lower leaf of every effective tiller was identified for measurement of leaf angle. First leaf after the flag leaf of the tiller was considered as upper leaf whereas the $3 \mathrm{rd}$ or 4 th leaf from an effective tiller was considered as middle leaves. In each case effective tillers of at least 15 plants were considered and measured using a protractor at panicle maturation stage of the plant and average leaf angles were calculated.

Disease scoring parameters. The disease related parameters include blast disease scoring, lesion number in leaves, lesion size, lesion type and sporulation center. Disease scoring was done in 15 days interval starting from 3rd day of the month of August each year. Scoring of the symptoms was done following the standardized disease scoring scale defined by IRRI ${ }^{20,21}$. The minimum score of ' 0 ' indicates of no disease and the maximum score of ' 9 ' indicates severe disease symptoms of coalesced eye shaped spots on the leaf surface leading to complete drying of the leaves (Fig. 1). The Percentage Disease Index (PDI) values were calculated every 15-day interval for 2 months after transplanting to the main field from nursery and finally Area Under Disease Progress Curve (AUDPC) values were calculated from PDI values. The leaves with distinguished disease symptoms were 


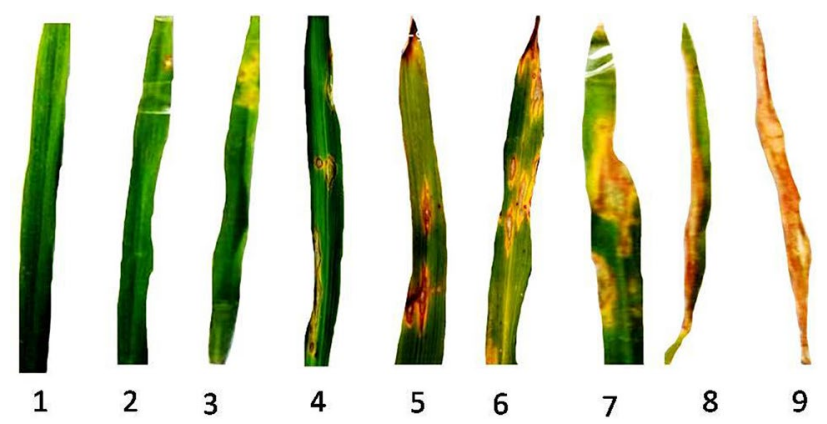

Figure 1. Pictorial representation of blast symptoms in the scale of 1 to 9 on rice leaves as evaluated for blast disease scoring.

considered for counting lesion number for each genotype in upper, middle and lower leaves. The typical spindle shaped brown lesions were counted in the leaves after 60 days of transplanting. Average lesion number was calculated from at least 50 such leaves for each case. The lesion area was calculated by measuring the length and breadth of the lesion using a millimeter scale. The lesion types were determined visually where the initial undefined yellow chlorotic lesions were marked as one where as fully matured brown necrotic lesion defined with white or gray center and brown lining in the periphery was defined as two. Average values for lesion type were calculated from at least 50 leaves for each genotype. Sporulation centre was considered based on the presence (one) and absence (zero) of the brown to black necrotic centers in the spindle shaped scars.

In vitro disease progression assay. The infected leaf samples from the field were collected and the fungus was purified by single spore isolation method on Water Agar media, cultured on Potato Dextrose Agar media. The fungal DNA was isolated and sequenced. The ITS sequences were analyzed by NCBI-BLAST for confirmation. The leaf cuttings of different genotypes were infected with the confirmed culture of purified Magnaporthe for in-vitro disease progression assay. The progression of the disease was measured by appearance of symptoms in time lapse photography and subsequent microscopic studies.

Nutritional parameters. The grains of selected aromatic genotypes (Fig. 2) were harvested, cleaned, dehusked and powdered. These powdered samples were used for estimation of all biochemical parameters.

Carbohydrate parameters. The Total Soluble Sugars (TSS) and Starch (STA) content from rice powder was estimated by following Anthrone method ${ }^{22}$. The final absorbance was measured at $630 \mathrm{~nm}$ and the concentrations of Starch (STA) and Total Soluble Sugars (TSS) were calculated from a standard reference plot of glucose (10$100 \mu \mathrm{g}$ ). The Reducing and Non-Reducing sugars (RES and NRS) from rice powder were estimated by following DNS method ${ }^{22}$. The absorbance was measured at $510 \mathrm{~nm}$. The concentration of Reducing and Non-Reducing Sugar (RES and NRS) were calculated from a standard reference plot of glucose (20-100 $\mu \mathrm{g})$. The Amylose content (AMY) from rice powder was estimated by following iodine-colorimetric method ${ }^{22}$. The absorbance was taken at $590 \mathrm{~nm}$. The concentration of amylose (\%) was calculated from a standard reference plot of pure amylose from Potato $(200-1000 \mu \mathrm{g})$. Amylopectin content (\%) was determined by subtracting the amylose content from total starch content.

Protein content. The protein content from the rice powder was estimated by Bradford method ${ }^{23}$. The absorbance was recorded at $595 \mathrm{~nm}$ wavelength. The concentration of protein percentage was calculated from a reference plot of Bovine Serum Albumin $(20-100 \mu \mathrm{g})$.

Resistant starch and glycemic index. The Resistant Starch (RS) of rice powder was estimated using Megazyme $\mathrm{kit}^{24}$. The absorbance was measured at $510 \mathrm{~nm}$. Amyloglucosidase was used as a standard enzyme. Resistant Starch content was calculated using the formulae $\mathrm{RS}=\Delta \mathrm{E} \times \mathrm{F} / \mathrm{W} \times 9.27$, where, $\Delta \mathrm{E}=$ Absorbance, $\mathrm{F} / \mathrm{W}=$ conversion of absorbance to micrograms $(100 \mu \mathrm{g})$. The Glycemic Index (GI) of rice powder was estimated using in vitro method following Kumar et al. ${ }^{24}$. The absorbance was measured at $510 \mathrm{~nm}$. Maltose $(200 \mathrm{mg})$ was used as standard carbohydrate. Average values were used to plot curves followed by computing the area under the curve (AUC). The Hydrolysis index (HI) for each rice variety was calculated by dividing AUC of sample by that of maltose and expressed in percentage. The predicted Glycemic Index was calculated using the following formula $(\mathrm{PGI})=39.71+(0.549 \times \mathrm{HI})$.

Antioxidant activity. The antioxidant activity of the powdered rice was estimated by DPPH method ${ }^{25}$. A stock solution of DPPH (2, 2-diphenyl-1-picrylhydrazyl) was made by dissolving $24 \mathrm{mg}$ in $100 \mathrm{ml}$ methanol and working solution was prepared by diluting $10 \mathrm{ml}$ of stock solution with $45 \mathrm{ml}$ of methanol. The absorbance was recorded at $515 \mathrm{~nm}$ using UV-VIS spectrophotometer, along with control (Methanol-0.5 $\mathrm{ml}+\mathrm{DPPH}-2.5 \mathrm{ml}$ ). The percentage of inhibition was expressed by putting the values into the formula: 


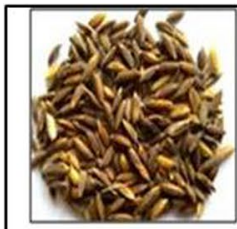

Kalturey

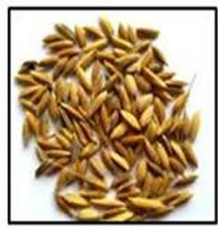

Dharmaphou

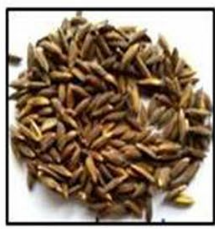

Kalonunia

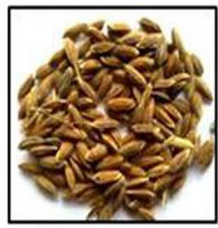

Dudheswar

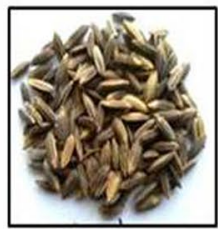

Chakhao Sel-3

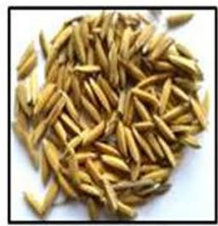

$\mathrm{T} 6 \mathrm{M}-3-3$

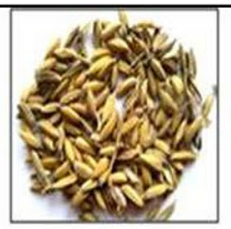

Tulsibhog

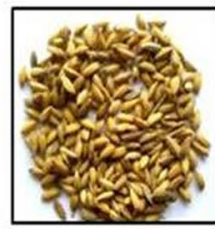

Radhatilak

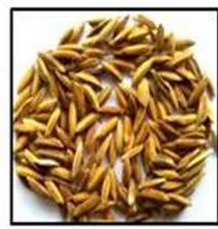

Rampha

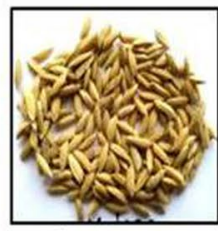

Kabra

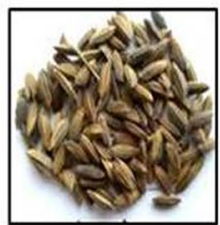

Chakhaos
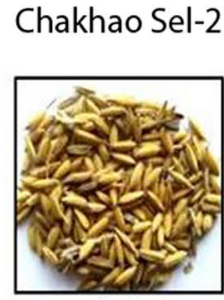

KNS-3-1(Uttar Sugandhi)

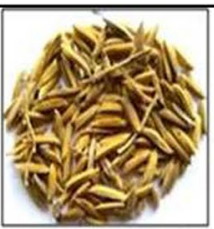

Sadanunia

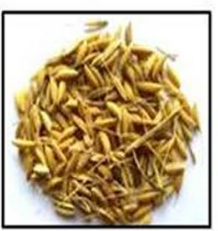

Kataribhog

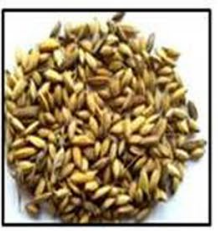

Konkanijoha

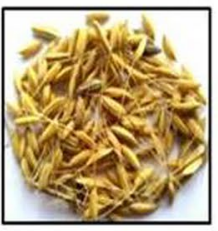

Sitalkuchi-1(A1-1)

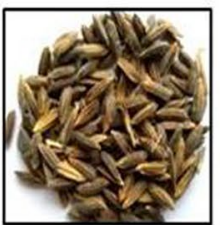

Chakhao Poiterin

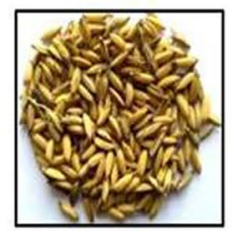

KNS-2-1-1

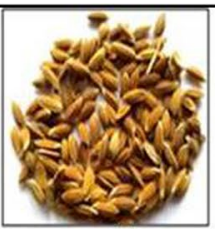

Rangakomal

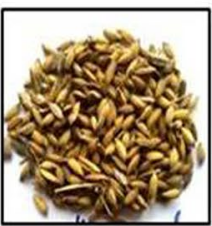

Radhunipagol

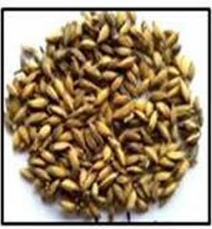

Kalokhasa

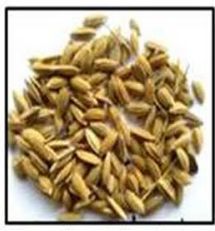

ChapkaChakhao

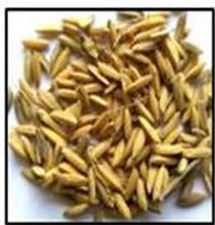

TSPM-3-1(TPUR-B-1)

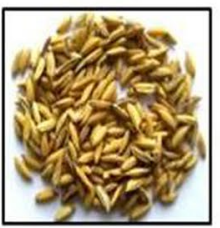

KNS-2B-S1

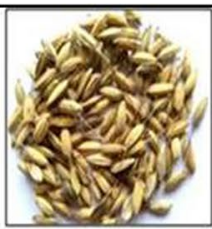

Kalshipa

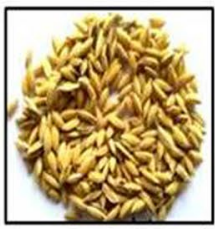

Gobindabhog

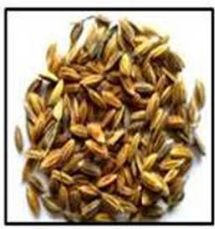

Dudhkalam

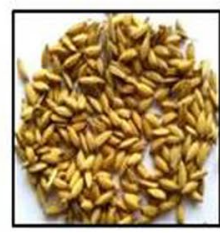

Dubarikomal
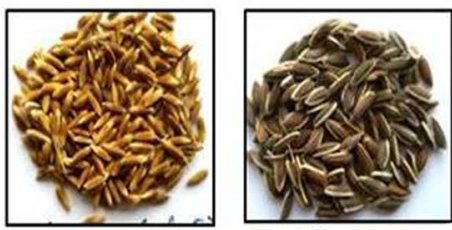

Chakhao Sel-I Chakhao Sampark

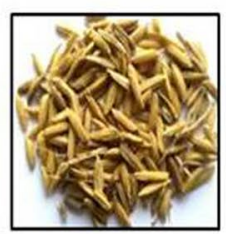

T4M-3-5

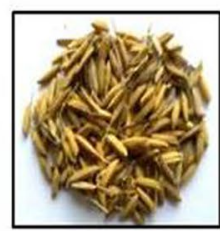

TSP6-M3-4

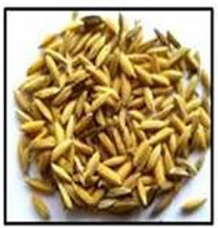

KNS-2-1

Figure 2. Paddy grain of the 35 aromatic landraces grown across sub-Himalayan Terai region of India. The low land region of north eastern India in the outer foothills of Himalaya and the north of the Indo-Gangetic plains is defined as the sub-Himalayan Terai region.

Percentage of Inhibition $(\%)=\frac{(\text { Absorbance of the control }- \text { Absorbance of the test samples }) \times 100}{\text { Absorbance of control }}$ 
The percentage of DPPH scavenging potential was plotted against the concentration of samples. The concentration of the sample necessary to decrease the DPPH concentration by $50 \%$ was obtained by interpolation from linear regression curve and denoted as $\mathrm{IC}_{50}$ value $(\mu \mathrm{g} / \mathrm{ml})$.

Aroma. The sensory test of rice aroma was performed by using $1.7 \% \mathrm{KOH}$ solution to the rice powder following the method described by Hien et al. ${ }^{26}$. Based on aroma the genotypes were ranked as (1) mild, (2) medium, (3) strong. In a given day only 10 samples were evaluated as handling more may cause biasness.

Genomic DNA isolation and genetic diversity analysis. The seeds of selected genotypes were geminated in water-soaked Petri plates under controlled condition. Genomic DNA was isolated from the young leaf tissue following the CTAB (cetyltrimethyl ammonium bromide) method with minor modifications. The quality of purified genomic DNA was estimated on $0.8 \%$ agarose gel electrophoresis. The DNA samples were later diluted with nuclease-free water to the concentration of $20 \mathrm{ng} / \mu \mathrm{l}$ and subjected to amplifications using SSR or ISSR markers ${ }^{27,28}$ and required PCR cocktail. The amplification of different alleles were scored as binary matrix where present or absent of an allele was denoted as 1 and 0 respectively in an agarose gel (1.5-3\%). Scoring was done on the basis of distinct, unambiguous and well resolved bands. Different parameters like heterozygosity index $(\mathrm{H})$, polymorphic information content (PIC), resolving power, discriminating power etc. were calculated using iMec server (https://irscope.shinyapps.io/iMEC/ ${ }^{29}$. The UPGMA based genetic clustering was performed by NTSYS-PC version $1.80^{30}$.

Allele mining for blast genes. Molecular screening, of the genotypes was performed for the presence of major blast resistant genes. The genotypes were differentiated on the basis of presence and/or absence of the above-mentioned blast resistant genes. PCR amplification was carried out in a $20 \mu \mathrm{l}$ reaction volume containing $2 \mu \mathrm{l}$ template DNA, (conc.) of Master Mix containing dNTP, $\mathrm{MgCl}_{2}$, Taq buffer and Taq DNA polymerase, forward and reverse primer and water. For scoring the marker genes the amplified PCR products were separated by electrophoresis in 1.5\% agarose gels stained with ethidium bromide along with DNA ladder (NEB). All PCR reactions were carried out twice for reproducibility.

Statistical analysis. Principal component analysis (PCA) and clustering. PCA have been carried out to identify the significant biochemical and disease related parameters. Based on PCA results, bi-plot analysis has been carried out to assess the impacts of different biochemical and phenotypic attributes respectively on different genotypes. The k-means non-hierarchical clustering algorithm was performed for grouping the rice genotypes based on different biochemical and phenotypic attributes. The number of clusters were determined using the gap statistic method. PCA and k-means non-hierarchical clustering techniques were carried out using R software version 3.5.1, Patched (2018-07-02 r74950) Platform: x86_64-w64-mingw32/x64 (64-bit) ${ }^{31}$. The correlation analysis between nutritional parameters and disease attributes were performed using SAS, version $9.3^{32}$.

Logistic regression analysis. Logistic regression modeling was carried out using IBM Statistical Package for the Social Sciences (SPSS trial version $20^{33}$ ) by considering only significant phenotypic characters except AUDPC as explanatory variable and presence or absence of blast disease as response variable where presence or absence of blast disease. If $\mathrm{X}_{1}, \mathrm{X}_{2}, \ldots \mathrm{X}_{\mathrm{p}}$ are $\mathrm{p}$ explanatory variables and if $\mathrm{Y}$ is the binary response variable taking value 0 and 1 for absence or presence of a particular attribute respectively, then the functional form of binary logistic regression equation is

$$
\pi=P(Y=1)=\frac{1}{\left(1+e^{-z}\right)}+\epsilon
$$

where $\pi$ is the probability of an event occurrence under consideration and $z=\beta_{0}+\beta_{1} X_{1}++\beta_{2} X_{2}+\ldots+\beta_{p} X_{p}$. Here, $\in$ is the error term. The parameters of the equation are generally estimated through iterative maximum likelihood estimation procedure. The goodness of fit of the model was determined by Hosmer and Lemeshow goodness of fit test.

Kendall's tau- $b$ correlation coefficient $\left(\tau_{b}\right)$. Kendall's tau-b correlation coefficient $\left(\tau_{\mathrm{b}}\right)$ was calculated using IBMSPSS (SPSS trial version $20^{33}$ ) which is a nonparametric measure of association between two qualitative variables. Kendall's tau-b correlation coefficient $\left(\tau_{\mathrm{b}}\right)$ was calculated between presence of blast resistant genes and tolerance of the blast disease in different genotypes where tolerance or susceptibility of blast disease was addressed based on clustering of the genotypes and AUDPC values.

\section{Results and discussion}

Field attributes of indigenous farmer's varieties collected from sub-HimalayanTerai region. A total of ninety-nine genotypes collected from different region of sub-Himalayan Terai region were subjected to purity breeding for at least five years and the pure lines were deposited to the Uttar Banga Krishi Viswavidyalaya (UBKV) rice repository. The performance of the genotypes for morphological and yield traits were assessed in the experimental plots. Majority of these genotypes are long duration, photosensitive, tall and have low yield potential (Table 1). The grains of these genotypes are predominantly long or short bold and very few genotypes have long to medium slender grains. Among the ninety-nine genotypes; twenty-seven were found to have mild to strong aroma among which only few genotypes exhibited very strong aroma. 'Tulaipanji', 'Gobindabhog', 


\begin{tabular}{|c|c|c|c|c|c|c|c|c|c|c|c|c|c|}
\hline Sl. no. & Farmers' varieties & $\mathrm{X} 1$ & $\mathrm{X} 2$ & $\mathrm{X} 3$ & $\mathrm{X} 4$ & $\mathrm{X} 5$ & X6 & $\mathrm{X} 7$ & $\mathrm{X8}$ & X9 & $\mathrm{X} 10$ & Grain type & Aroma \\
\hline 1. & Ayangleima Phou & 73.33 & 11.35 & 128.50 & 24.08 & 232.50 & 7.32 & 2.77 & 2.64 & 2.57 & 1.78 & Long bold & No aroma \\
\hline 2. & Baigon Macchua & 129.78 & 14.05 & 139.75 & 23.95 & 208.75 & 4.11 & 2.38 & 1.73 & 2.26 & 1.60 & Short bold & No aroma \\
\hline 3. & Betho & 137.95 & 22.20 & 138.00 & 23.78 & 140.25 & 5.64 & 2.51 & 2.25 & 2.34 & 2.01 & Short bold & No aroma \\
\hline 4. & Beto & 115.99 & 26.90 & 132.75 & 23.89 & 110.75 & 5.69 & 1.96 & 2.90 & 2.45 & 1.69 & Short bold & No aroma \\
\hline 5. & Binni & 127.85 & 20.00 & 125.25 & 23.54 & 118.05 & 7.19 & 2.21 & 3.25 & 2.69 & 1.56 & Long slender & No aroma \\
\hline 6. & Birali & 125.24 & 21.20 & 131.75 & 26.74 & 135.20 & 5.77 & 2.19 & 2.63 & 2.05 & 1.17 & Short bold & No aroma \\
\hline 7. & Birali-Selection & 146.65 & 14.90 & 138.25 & 24.57 & 152.00 & 7.45 & 2.20 & 3.39 & 2.93 & 2.26 & Long slender & No aroma \\
\hline 8. & Boichi & 136.04 & 13.90 & 134.00 & 26.08 & 113.45 & 5.84 & 2.83 & 2.06 & 2.22 & 1.93 & Short bold & No aroma \\
\hline 9. & Bonnidhan & 119.45 & 16.55 & 135.75 & 24.95 & 101.80 & 6.23 & 2.51 & 2.48 & 2.91 & 2.65 & Long bold & No aroma \\
\hline 10. & Chakhao Amubi & 125.60 & 14.35 & 128.75 & 23.56 & 132.95 & 6.38 & 2.61 & 2.44 & 2.41 & 2.04 & Long bold & No aroma \\
\hline 11. & Chakhao Angangbi & 124.56 & 20.50 & 117.75 & 23.11 & 105.55 & 7.16 & 2.71 & 2.64 & 2.50 & 1.61 & Long bold & No aroma \\
\hline 12. & Chakhao Poireiton & 136.61 & 15.75 & 119.25 & 26.65 & 175.75 & 6.44 & 2.40 & 2.68 & 2.49 & 1.54 & Long bold & Strong \\
\hline 13. & Chakhao Sampark & 129.07 & 13.75 & 120.00 & 25.25 & 132.08 & 7.15 & 2.73 & 2.62 & 2.70 & 1.55 & Long bold & Medium \\
\hline 14. & Chakhao selection-1 & 127.80 & 21.90 & 133.25 & 22.41 & 166.85 & 5.96 & 2.59 & 2.30 & 2.28 & 2.06 & Short bold & Medium \\
\hline 15. & Chakhao selection-2 & 111.32 & 11.50 & 119.50 & 22.95 & 162.14 & 6.52 & 2.34 & 2.79 & 2.58 & 1.88 & Long slender & Medium \\
\hline 16. & Chakhao selection-3 & 118.29 & 19.45 & 120.50 & 22.66 & 188.25 & 5.70 & 2.72 & 2.10 & 2.31 & 1.72 & Short bold & Medium \\
\hline 17. & Chapka Chakhao & 84.77 & 18.45 & 126.75 & 20.06 & 150.30 & 6.50 & 2.27 & 2.86 & 2.39 & 2.11 & Long slender & Mild \\
\hline 18. & Dharamphou & 96.68 & 13.60 & 129.50 & 24.71 & 213.00 & 6.79 & 2.43 & 2.79 & 3.89 & 2.86 & Long slender & Medium \\
\hline 19. & Dhyapa & 127.24 & 23.60 & 132.75 & 24.27 & 174.85 & 5.80 & 2.70 & 2.15 & 2.86 & 2.31 & Short bold & No aroma \\
\hline 20. & Dubarikomal & 141.73 & 19.10 & 124.75 & 26.17 & 134.20 & 6.15 & 2.63 & 2.34 & 2.76 & 2.30 & Long bold & Medium \\
\hline 21. & Dudhkalam Motajosawa & 126.96 & 20.35 & 140.25 & 25.06 & 129.30 & 6.29 & 2.37 & 2.65 & 2.80 & 2.71 & Long bold & No aroma \\
\hline 22. & Dudhkalam & 141.77 & 15.70 & 140.50 & 28.18 & 161.40 & 6.16 & 2.44 & 2.52 & 2.45 & 2.21 & Long bold & Mild \\
\hline 23. & Dudhkalam-9 & 111.73 & 18.35 & 139.75 & 28.44 & 137.90 & 7.38 & 2.55 & 2.89 & 2.71 & 1.51 & Long slender & No aroma \\
\hline 24. & Dudheswar & 124.62 & 20.00 & 127.25 & 25.37 & 118.35 & 6.31 & 2.57 & 2.46 & 2.44 & 1.43 & Long bold & Medium \\
\hline 25. & Dudheswar-AD & 123.04 & 15.20 & 135.75 & 28.35 & 195.55 & 6.61 & 1.94 & 3.41 & 2.05 & 1.49 & Long slender & No aroma \\
\hline 26. & Fudugey & 139.22 & 29.85 & 130.00 & 25.64 & 98.65 & 6.08 & 2.22 & 2.74 & 2.52 & 1.64 & Long bold & No aroma \\
\hline 27. & Gobindabhog & 124.05 & 15.20 & 138.00 & 26.27 & 231.95 & 4.32 & 1.71 & 2.53 & 1.34 & 1.36 & Short bold & Strong \\
\hline 28. & Jaldhyapa-2 & 132.45 & 22.55 & 138.00 & 25.70 & 149.45 & 5.93 & 2.75 & 2.16 & 2.63 & 1.81 & Short bold & No aroma \\
\hline 29. & Jaldhyapa-3 & 127.93 & 22.85 & 140.25 & 24.71 & 102.80 & 6.04 & 2.70 & 2.24 & 5.47 & 3.77 & Long bold & No aroma \\
\hline 30. & Jaldhyapa-AD & 132.05 & 24.10 & 138.00 & 27.34 & 134.60 & 6.89 & 2.85 & 2.42 & 3.35 & 2.36 & Long bold & No aroma \\
\hline 31. & Jasawa-AD & 121.90 & 14.90 & 122.00 & 26.80 & 157.10 & 6.06 & 2.79 & 2.17 & 2.85 & 2.23 & Long bold & No aroma \\
\hline 32. & Jashyoya & 132.86 & 14.95 & 138.75 & 25.27 & 108.35 & 6.03 & 2.78 & 2.17 & 4.38 & 3.51 & Long bold & No aroma \\
\hline 33. & Jhapaka & 155.09 & 15.35 & 111.00 & 25.22 & 112.20 & 6.58 & 2.49 & 2.64 & 2.31 & 2.08 & Long bold & No aroma \\
\hline 34. & Jonroi Buna & 169.76 & 19.00 & 125.50 & 22.79 & 139.85 & 5.59 & 2.74 & 2.04 & 2.51 & 1.71 & Short bold & No aroma \\
\hline 35. & Kabra & 120.19 & 26.60 & 136.00 & 26.37 & 140.05 & 6.13 & 2.10 & 2.92 & 2.15 & 1.72 & Long slender & Strong \\
\hline 36. & Kagey & 147.70 & 23.75 & 125.75 & 32.31 & 200.15 & 4.66 & 2.87 & 1.62 & 2.08 & 1.72 & Short bold & No aroma \\
\hline 37. & Kaike & 110.85 & 20.95 & 137.00 & 24.86 & 112.50 & 6.00 & 2.64 & 2.27 & 2.77 & 1.88 & Short bold & No aroma \\
\hline 38. & Kaloboichi & 103.29 & 23.90 & 136.00 & 21.43 & 105.95 & 6.63 & 2.27 & 2.92 & 2.42 & 1.71 & Long slender & No aroma \\
\hline 39. & Kalodhyapa & 120.92 & 24.95 & 136.00 & 25.62 & 151.00 & 6.10 & 2.61 & 2.34 & 2.34 & 1.84 & Long bold & No aroma \\
\hline 40. & Kalojeera & 130.62 & 20.95 & 136.00 & 27.50 & 146.75 & 4.22 & 2.36 & 1.79 & 1.44 & 1.41 & Shot bold & Strong \\
\hline 41. & Kalokhasa & 133.22 & 21.00 & 128.50 & 28.07 & 167.75 & 4.23 & 2.90 & 1.46 & 1.05 & 0.99 & Short bold & Mild \\
\hline 42. & Kalonunia & 137.64 & 13.30 & 137.25 & 25.29 & 114.65 & 5.30 & 1.96 & 2.70 & 1.56 & 0.95 & Medium slender & Strong \\
\hline 43. & Kalshipa & 116.82 & 24.00 & 134.50 & 25.14 & 140.70 & 5.91 & 2.36 & 2.50 & 2.25 & 2.03 & Short bold & Mild \\
\hline 44. & Kalturey & 157.41 & 13.90 & 119.00 & 25.31 & 98.85 & 6.02 & 1.99 & 3.03 & 1.41 & 1.33 & Long slender & Strong \\
\hline 45. & KashiyaBinni & 130.33 & 21.04 & 133.20 & 25.19 & 133.52 & 6.45 & 2.57 & 2.51 & 2.61 & 1.94 & Long bold & No aroma \\
\hline 46. & Kataribhog & 133.40 & 21.50 & 129.75 & 26.75 & 134.20 & 5.76 & 1.96 & 2.94 & 1.79 & 1.97 & Medium slender & Medium \\
\hline 47. & Kauka-Selection & 128.26 & 21.00 & 136.25 & 23.56 & 115.30 & 5.02 & 2.54 & 1.98 & 2.68 & 2.24 & Short bold & No aroma \\
\hline 48. & Khaiyamdhan & 135.75 & 25.50 & 133.50 & 25.73 & 105.95 & 6.45 & 2.59 & 2.49 & 2.65 & 2.24 & Long bold & No aroma \\
\hline 49. & Kharadhan & 145.55 & 17.35 & 131.75 & 29.52 & 211.60 & 6.36 & 2.51 & 2.53 & 4.38 & 2.83 & Long bold & No aroma \\
\hline 50. & KonkoniJoha & 128.05 & 19.75 & 131.25 & 29.01 & 273.52 & 4.36 & 2.43 & 1.79 & 1.42 & 1.31 & Short bold & Strong \\
\hline 51. & Ladu & 137.59 & 20.35 & 128.00 & 25.93 & 148.55 & 5.11 & 2.69 & 1.90 & 2.43 & 1.77 & Short bold & No aroma \\
\hline 52. & Maitee & 150.95 & 14.05 & 116.50 & 30.24 & 163.05 & 4.45 & 2.66 & 1.67 & 2.10 & 1.36 & Short bold & No aroma \\
\hline 53. & Malbati & 147.27 & 18.80 & 120.75 & 27.22 & 167.10 & 6.14 & 1.60 & 3.84 & 2.03 & 1.41 & Long bold & No aroma \\
\hline 54. & Malshira & 118.55 & 21.20 & 131.00 & 26.64 & 137.80 & 6.12 & 2.22 & 2.76 & 2.20 & 2.59 & Long slender & No aroma \\
\hline 55. & Mangamuthi & 161.53 & 16.85 & 134.50 & 27.41 & 163.40 & 6.75 & 3.04 & 2.22 & 3.27 & 2.28 & Long bold & No aroma \\
\hline 56. & Pahariboichi & 117.58 & 23.55 & 131.75 & 24.87 & 158.40 & 5.90 & 2.55 & 2.31 & 2.04 & 2.04 & Short bold & No aroma \\
\hline \multicolumn{14}{|c|}{ Continued } \\
\hline
\end{tabular}




\begin{tabular}{|c|c|c|c|c|c|c|c|c|c|c|c|c|c|}
\hline Sl. no. & Farmers' varieties & $\mathrm{X} 1$ & $\mathrm{X} 2$ & $\mathrm{X} 3$ & $\mathrm{X} 4$ & $\mathrm{X} 5$ & X6 & $\mathrm{X} 7$ & $\mathrm{X} 8$ & X9 & $\mathrm{X} 10$ & Grain type & Aroma \\
\hline 57. & Pahariboichi-Selection & 128.91 & 20.65 & 116.25 & 26.28 & 164.05 & 5.46 & 2.38 & 2.29 & 2.34 & 1.86 & Short bold & No aroma \\
\hline 58. & PanikuthiShyamlal & 134.96 & 21.95 & 140.75 & 26.55 & 149.15 & 6.09 & 2.31 & 2.64 & 3.34 & 2.00 & Long bold & No aroma \\
\hline 59. & Phoolpakari & 116.36 & 25.25 & 134.50 & 24.11 & 141.45 & 5.76 & 1.95 & 2.95 & 1.49 & 1.39 & Medium slender & No aroma \\
\hline 60. & Phoolpakari-1 & 116.86 & 18.50 & 129.00 & 22.28 & 129.35 & 5.21 & 1.86 & 2.80 & 1.82 & 1.34 & Medium slender & No aroma \\
\hline 61. & Phorenmubi & 123.79 & 15.05 & 120.75 & 26.41 & 140.25 & 7.07 & 2.14 & 3.30 & 2.64 & 1.61 & Long slender & No aroma \\
\hline 62. & Radhatilak & 127.65 & 14.95 & 129.75 & 24.70 & 241.75 & 4.96 & 1.94 & 2.56 & 1.50 & 1.28 & Short bold & medium \\
\hline 63. & Radhatilak-2 & 119.84 & 16.40 & 136.50 & 24.31 & 161.50 & 4.34 & 1.93 & 2.25 & 1.12 & 1.02 & Short bold & No aroma \\
\hline 64. & Radhunipagol & 144.08 & 21.75 & 133.25 & 25.97 & 173.75 & 4.57 & 1.92 & 2.38 & 1.56 & 1.48 & Short bold & Strong \\
\hline 65. & Rampha & 139.84 & 16.45 & 132.75 & 25.35 & 122.65 & 6.05 & 1.98 & 3.06 & 2.15 & 2.16 & Long slender & Strong \\
\hline 66. & Rongakomal & 134.55 & 20.75 & 129.50 & 24.54 & 156.75 & 5.11 & 2.82 & 1.81 & 2.58 & 2.05 & Short slender & Medium \\
\hline 67. & Sada Mala & 101.70 & 17.40 & 127.75 & 24.66 & 152.10 & 6.37 & 2.14 & 2.98 & 2.03 & 1.55 & Long slender & No aroma \\
\hline 68. & Sadanunia & 105.06 & 13.80 & 91.75 & 28.72 & 124.40 & 7.81 & 2.97 & 2.63 & 2.11 & 1.85 & Extra-long slender & Medium \\
\hline 69. & Sadabhatkalo & 131.59 & 21.35 & 138.50 & 24.91 & 187.80 & 6.72 & 2.52 & 2.67 & 2.58 & 2.20 & Long bold & No aroma \\
\hline 70. & Satia & 124.37 & 23.30 & 131.25 & 24.26 & 121.25 & 5.60 & 2.25 & 2.49 & 2.29 & 2.14 & Short bold & No aroma \\
\hline 71. & Seshphal & 83.14 & 14.95 & 95.75 & 24.09 & 166.70 & 5.30 & 1.88 & 2.82 & 1.88 & 1.89 & Medium slender & No aroma \\
\hline 72. & Sitalkuchi-1 (A-1-1) & 112.97 & 12.35 & 132.25 & 28.14 & 128.95 & 5.31 & 2.86 & 1.86 & 2.50 & 2.20 & Short bold & Medium \\
\hline 73. & Sitalkuchi-2 & 122.04 & 13.85 & 136.75 & 23.90 & 166.50 & 5.80 & 2.60 & 2.23 & 2.63 & 2.12 & Short bold & No aroma \\
\hline 74. & Sitalkuchi-3 & 128.22 & 23.20 & 130.50 & 24.68 & 147.02 & 6.13 & 2.91 & 2.11 & 2.97 & 2.84 & Long bold & No aroma \\
\hline 75. & Sitalkuchi-5 & 105.79 & 20.75 & 130.50 & 27.00 & 110.90 & 5.58 & 2.45 & 2.28 & 2.19 & 1.94 & Short bold & No aroma \\
\hline 76. & Sitalkuchi-6 & 122.95 & 19.50 & 136.50 & 25.78 & 107.17 & 6.49 & 2.36 & 2.75 & 2.38 & 1.61 & Long bold & No aroma \\
\hline 77. & Tarai Research Society-1 & 122.80 & 21.00 & 137.00 & 25.35 & 151.35 & 5.70 & 2.62 & 2.18 & 2.41 & 1.35 & Short bold & No aroma \\
\hline 78. & Tarai Research Society-2 & 112.75 & 23.65 & 126.50 & 23.71 & 98.50 & 6.31 & 2.39 & 2.64 & 2.33 & 1.59 & Long bold & No aroma \\
\hline 79. & Tarai Research Society-3 & 117.18 & 15.95 & 136.50 & 24.13 & 267.08 & 4.18 & 2.36 & 1.77 & 2.07 & 1.96 & Short bold & No aroma \\
\hline 80. & Tarai Research Society- 4 & 123.01 & 14.25 & 134.00 & 25.09 & 135.40 & 5.21 & 1.87 & 2.79 & 1.76 & 1.60 & Medium slender & No aroma \\
\hline 81. & Tarapakari & 118.59 & 22.00 & 136.50 & 25.53 & 198.15 & 4.88 & 1.82 & 2.68 & 2.07 & 1.61 & Medium slender & No aroma \\
\hline 82. & Tarapakari-Selection & 131.62 & 21.90 & 132.50 & 27.46 & 177.70 & 4.70 & 2.47 & 1.90 & 2.29 & 2.22 & Short bold & No aroma \\
\hline 83. & Thuri & 114.73 & 16.60 & 141.00 & 21.76 & 282.10 & 6.19 & 2.33 & 2.66 & 2.43 & 2.06 & Long bold & No aroma \\
\hline 84. & Tulaipanji & 118.47 & 27.75 & 131.50 & 24.66 & 97.85 & 6.18 & 1.84 & 3.36 & 1.44 & 0.83 & Long slender & Strong \\
\hline 85. & Tulsibhog & 136.78 & 25.10 & 131.50 & 24.11 & 123.85 & 5.44 & 2.01 & 2.71 & 1.47 & 1.17 & Short bold & Strong \\
\hline 86. & Tulsimukul & 141.12 & 16.30 & 138.00 & 30.61 & 170.30 & 4.45 & 2.19 & 2.03 & 1.57 & 1.68 & Short bold & No aroma \\
\hline 87. & Uttar Banga Loca-3 & 127.68 & 21.00 & 139.75 & 27.23 & 178.90 & 5.71 & 2.43 & 2.35 & 2.75 & 2.16 & Short bold & No aroma \\
\hline 88. & Uttar Banga Local-10 & 109.93 & 15.00 & 118.50 & 26.21 & 124.00 & 8.08 & 2.00 & 4.04 & 3.17 & 1.79 & Extra-long slender & No aroma \\
\hline 89. & Uttar Banga Local-11 & 131.72 & 15.60 & 133.75 & 25.64 & 215.10 & 5.39 & 2.35 & 2.29 & 2.01 & 2.10 & Short bold & No aroma \\
\hline 90. & Uttar Banga Local-13 & 135.50 & 19.75 & 133.75 & 25.40 & 176.50 & 4.36 & 2.07 & 2.11 & 1.56 & 1.80 & Short bold & No aroma \\
\hline 91. & Uttar Banga Local-14 & 128.00 & 19.05 & 136.75 & 23.04 & 170.95 & 7.80 & 2.29 & 3.41 & 3.37 & 2.09 & Extra-long slender & No aroma \\
\hline 92. & Uttar Banga Local-15 & 126.86 & 22.25 & 133.50 & 26.38 & 106.60 & 5.61 & 2.43 & 2.31 & 2.59 & 2.13 & Short bold & No aroma \\
\hline 93. & Uttar Banga Local-17 & 131.27 & 21.55 & 133.00 & 29.74 & 206.25 & 6.21 & 2.03 & 3.06 & 2.58 & 1.69 & Long slender & No aroma \\
\hline 94. & Uttar Banga Local-18 & 89.37 & 12.40 & 140.00 & 24.76 & 139.50 & 7.05 & 1.80 & 3.92 & 1.81 & 1.73 & Long slender & No aroma \\
\hline 95. & Uttar Banga Local-2-AD & 121.49 & 21.25 & 130.50 & 25.66 & 110.45 & 6.08 & 2.26 & 2.69 & 1.82 & 1.48 & Long bold & No aroma \\
\hline 96. & Uttar Banga Local-3-1 & 140.96 & 20.65 & 136.00 & 26.31 & 98.85 & 5.61 & 2.74 & 2.05 & 2.22 & 2.03 & Short bold & No aroma \\
\hline 97. & Uttar Banga Local-5 & 113.13 & 13.40 & 137.50 & 24.96 & 254.17 & 4.29 & 2.68 & 1.60 & 2.09 & 1.43 & Short bold & No aroma \\
\hline 98. & Uttar Banga Local-6 & 115.74 & 20.25 & 136.25 & 25.87 & 108.45 & 6.33 & 2.55 & 2.48 & 2.45 & 1.46 & Long bold & No aroma \\
\hline 99. & Uttar Banga Local-9 & 131.33 & 25.20 & 132.50 & 29.09 & 142.90 & 6.66 & 2.33 & 2.86 & 2.22 & 1.84 & Long bold & No aroma \\
\hline 100. & KNS-2'-1 & 108.69 & 16.77 & 118.45 & 22.55 & 168.26 & 5.25 & 1.81 & 2.90 & 1.51 & 2.14 & Medium slender & Strong \\
\hline 101. & $\begin{array}{l}\text { KNS-3'-1 Uttar Sugandhi } \\
\text { (IET 24616) }\end{array}$ & 107.82 & 22.05 & 120.50 & 25.00 & 179.73 & 5.04 & 1.87 & 2.69 & 1.48 & 3.33 & Medium slender & Strong \\
\hline 102. & KNS-2-1-1 & 116.51 & 18.10 & 119.35 & 18.80 & 153.47 & 5.30 & 1.83 & 2.89 & 1.53 & 1.94 & Medium slender & Strong \\
\hline 103. & KNS-2B-S1 & 111.92 & 20.40 & 123.45 & 16.85 & 161.29 & 5.55 & 1.92 & 2.89 & 1.60 & 2.05 & Medium slender & Strong \\
\hline 104. & T4M-3-5 & 85.80 & 17.15 & 120.00 & 21.00 & 112.00 & 6.24 & 2.19 & 2.85 & 1.73 & 3.06 & Long slender & Strong \\
\hline 105. & TSP6-M3-4 & 81.83 & 21.73 & 122.00 & 23.00 & 107.40 & 6.31 & 2.35 & 2.80 & 1.76 & 3.22 & Long slender & Strong \\
\hline 106. & TPUR-B-1 (IET 28104) & 92.12 & 20.62 & 118.00 & 23.40 & 120.40 & 6.45 & 2.13 & 3.03 & 1.71 & 2.13 & Long slender & Strong \\
\hline 107. & T6M-3-3 & 80.40 & 21.22 & 117.00 & 23.60 & 115.60 & 6.27 & 2.21 & 2.84 & 1.59 & 2.69 & Long slender & Strong \\
\hline
\end{tabular}

Table 1. Mean performance of ninety-nine indigenous farmers' varieties of rice (Oryza sativa L.) for yield and its attributes. $X 1$ : plant height $(\mathrm{cm}), X 2$ : panicle no./plant, $X 3$ : days to $50 \%$ flowering, $X 4$ : panicle length $(\mathrm{cm})$, $X 5$ : filled grain/panicle, $X 6$ : decorticated grain length $(\mathrm{mm}), X 7$ : decorticated grain width $(\mathrm{mm}), X 8: \mathrm{L}: B$ ratio, X9: 100-seed weight (g), X10: yield (t/ha). 
'Kalonunia,' 'Radhunipagol', 'Konkanijoha, 'Chakhao' etc. are traditionally known very important cultivars in northern Bengal as well as in different states of' north eastern India. Especially 'Kalonunia' and 'Tulaipanji' are two genotypes of great traditional value in Terai and Duars region and have excellent market potential. At UBKV eight photo-insensitive lines of these two cultivars have been developed keeping the fragrance intact using mutation breeding and selection programs ${ }^{34}$. Since the aroma is the most important criteria in terms of consumer preference of these genotypes, only thirty-five aromatic genotypes (twenty-seven farmers genotypes and eight UBKV developed lines from these genotypes) from total one hundred and seven genotypes (Supplementary information $1 \mathrm{a}, 1 \mathrm{~b}$, Table 1) were chosen for furthers studies. The detailed description of the selected genotypes along with their origin, ecology, place of collection and yield potential is given in Table 2.

Genetic diversity of indigenous aromatic cultivars using microsatellite markers. A total of forty-two microsatellite markers (thirty-two SSR and ten ISSR) were used for evaluating genetic diversity of the selected aromatic genotypes (Table 3) 27,28 . A total of 45 alleles were detected using SSR markers and 30 alleles were detected using purine rich ISSR markers. Except RM469 all the SSR markers were found to be polymorphic in nature. Percentage polymorphism varied between $14.29 \%$ for RM460 to $97.14 \%$ for RM108 and RM434. Polymorphism Information Content (PIC) value varied from 0.052 (RM434) to 0.375(RM288) with highest Heterozygosity index (5.00) (Table 4). RM 1, RM23, RM 38, RM 250, RM 314, RM 3134, RM 3872, RM 6250 etc. exhibited good resolving and discriminatory power among the SSR markers. All the purine rich ISSR Markers exhibited very good discriminatory power and high PIC ranging from 0.295 to 0.374 and proved to be more effective for diversity analysis. Based on the microsatellite marker analysis genetic clustering was performed using NTSYS software (Fig. 3, Supplementary information 2a, 2b). Clustering using UPGMA method grouped the total thirty-five accessions into three distinct clusters. The distance coefficient of these clusters ranged from 0.45 to 0.85 . Cluster I consisted of two subgroups, subgroup I has five genotypes i.e. 'Tulaipanji', 'Kalshipa', 'Dubarikomal', 'Dharmaphou' and 'Dudheswar', majority of these genotypes are photo sensitive, long duration, tall, lodging susceptible having low yield potential (2-2.5 t/ha). Seven genotypes, 'Radhatilak', 'Kalturey', 'Sadanunia, 'Tulsibhog', 'Kataribhog, 'A-1-1' and 'Rangakomal' constitute Sub group II. Majority of these genotypes exhibits very low yield potential (1.5-2 t/ha). The second cluster constitutes of sixteen genotypes amongst them four genotypes is black rice introduced from north eastern Manipur state and the remaining genotypes, 'Kalonunia, 'Gobindabhog, 'Radhunipagol', 'Konkanijoha,' 'Rampha' etc. are known for their excellent aroma. The selections from 'Kalonunia' with strong aroma are found in this cluster. The third cluster is majorly comprised of comparatively high-yielding photo insensitive lines of 'Tulaipanji' and 'Kalonunia' along with two 'Chakhao' cultivars. Yield potential of this cluster varied from 3.5 to $4 \mathrm{t} / \mathrm{ha}$.

The evaluation of genetic diversity for characterization of these heirloom cultivars is necessary and relatively easy due to the availability of nearly saturated molecular map in case of rice ${ }^{27,28,35}$. SSRs are interspersed throughout the genome and known as mono-locular, co-dominant, highly informative and easy to analyze marker system where as ISSR markers relies on inter tandem repeats of di, tri, tetra or penta nucleotides found at microsatellite loci and gives an array of amplified products. Purine rich ISSR markers have been proved to be very useful in determining genetic relationship between diverse population ${ }^{36-38}$. In the present study we have performed genetic diversity analysis of locally grown scented landraces using ISSR and SSR markers and based on the results the cultivars were clustered. The results of the marker analysis emphasize that each of these cultivars are distinct from the other and the population under study is genetically diverse. It is noteworthy that the marker-based clustering grouped the genotypes according to the yield potential which proves the accuracy and usefulness of microsatellite marker system in determining purity and phylogeny of the germplasm. This natural population of aromatic cultivars with such wide genetic variability may prove to be good resources for excellent quality traits and disease tolerance. These cultivars were further analyzed for the grain quality attributes and tolerance to destructive blast disease.

Analysis of nutritional variability in selected rice genotypes. Nutritional parameters like Total Soluble Sugar (TSS), Reducing Sugar (RES), Non- Reducing Sugar (NRS), Starch (STA), Amylose (AMY), Resistant Starch (RS), Glycemic Index (GI), Protein (PRO), Antioxidant properties (ANT) and Aroma (ARO) has been measured for thirty-five selected genotypes (Table 5). The TSS content of the genotypes varied from $88.89 \%$ ('Chakhao Sampark') to $37.44 \%$ ('Tulsibhog') whereas average starch content of these genotypes ranged from $85.45 \%$ ('Sadanunia') to $45.86 \%$ ('Konkanijoha'). The wide variability in carbohydrate content prompted us to measure the amylose content of these genotypes as the ratio of amylose and amylopectin content determines the stickiness and flakiness of the rice after cooking. Higher amylose content was found in KNS-2-1-1 (31.27\%), 'Tulsibhog' (29.10\%) and 'Kabra' (28.77\%) etc. whereas low amylose was found in genotypes like 'Chakhao' selections, 'Kalojeera, 'Konkanijoha', 'Gobindabhog, 'Kalturey' etc. Good resistant starch (RS) content in 'Kataribhog' (2.25\%), 'Chakhao Sampark' (2.22\%), and KNS-2-1-1 (2.11\%) were found. Although most of the genotypes showed an average GI value of more than $60 \%$, few cultivars like 'Dubarikomal (54.77\%), 'A-1-1' (58.67\%), 'KNS2B-S1' (55.08\%), 'TPUR-B-1-IET 28104' (55.63\%) exhibited quite encouraging low glycemic index. Powdered 'Kataribhog' grains were found to have remarkable GI, as low as $45.72 \%$ by initial analysis which is even lower than the GI of recommended rice varieties for diabetic patients ${ }^{39}$. The protein content of the genotypes was varying from $4.11 \%$ in 'Rampha' and was highest in $9.47 \%$ in 'Dharmaphou'. When the total antioxidant activity was evaluated for powdered grains of these genotypes all the genotypes with black pericarp has exhibited the highest antioxidant potential ('Chakhao sel-2', 'Chakhao sel-3', 'Chakhao Sampark', 'Chakhao Poiterin' and 'Kalshipa'). Apart from black genotypes four indigenous cultivars 'Sadanunia', 'Kabra,' 'Kalturey', 'Rangakomal' have exhibited very high antioxidant activity comparable to the black varieties. All the varieties exhibited good to very good aroma. 


\begin{tabular}{|c|c|c|c|c|c|c|}
\hline Sl no. & Name of the genotype & $\begin{array}{l}\text { Description of the } \\
\text { genotype }\end{array}$ & Origin & Ecology & Parentage & $\begin{array}{l}\text { Place of collection/ } \\
\text { source of the seed }\end{array}$ \\
\hline 1. & Tulaipanji & $\begin{array}{l}\text { Photo-period sensitive, } \\
\text { long duration, medium tall, } \\
\text { medium slender grain with } \\
\text { long awn, lodging suscep- } \\
\text { tible, low yield potential } \\
(1.5-2.0 \mathrm{t} / \mathrm{ha})\end{array}$ & $\begin{array}{l}\text { Raiganj sub-division, Uttar } \\
\text { dinajpur district, West } \\
\text { Bengal }\end{array}$ & Medium or low land & Landrace & $\begin{array}{l}\text { Uttar Dinajpur KVK, West } \\
\text { Bengal }\end{array}$ \\
\hline 2. & Radhatilak & $\begin{array}{l}\text { Photo-period sensitive, long } \\
\text { duration, tall, short bold } \\
\text { grain, lodging suscepti- } \\
\text { ble, low yield potential } \\
(3.0-3.5 \text { t/ha) }\end{array}$ & $\begin{array}{l}\text { Northern part of West } \\
\text { Bengal }\end{array}$ & Medium or low land & Landrace & $\begin{array}{l}\text { Tarai Research Society, } \\
\text { Alipurduar, West Bengal }\end{array}$ \\
\hline 3. & Kalshipa & $\begin{array}{l}\text { Photo-period sensitive, long } \\
\text { duration, tall, bold grain, } \\
\text { lodging susceptible, low } \\
\text { yield potential }(2.5-3.0 \mathrm{t} / \mathrm{ha})\end{array}$ & $\begin{array}{l}\text { Northern part of West } \\
\text { Bengal }\end{array}$ & Medium or low land & Landrace & $\begin{array}{l}\text { Tarai Research Society, } \\
\text { Alipurduar, West Bengal }\end{array}$ \\
\hline 4. & Rangakomal & $\begin{array}{l}\text { Photo-period sensitive, long } \\
\text { duration, tall, bold grain, } \\
\text { brown-red grain husk, lodg- } \\
\text { ing susceptible, low yield } \\
\text { potential }(2.0-2.5 \mathrm{t} / \mathrm{ha})\end{array}$ & $\begin{array}{l}\text { Lower-eastern part of } \\
\text { Assam }\end{array}$ & Medium or low land & Landrace & $\begin{array}{l}\text { ICAR-CPCRI- Kahikuchi, } \\
\text { Kamrup, Assam }\end{array}$ \\
\hline 5. & Sadanunia & $\begin{array}{l}\text { Photo-period sensitive, } \\
\text { medium duration, medium } \\
\text { tall, long slender grain with } \\
\text { long awn, low yield poten- } \\
\text { tial (1.5-2.0 t/ha) }\end{array}$ & $\begin{array}{l}\text { Northern part of West } \\
\text { Bengal }\end{array}$ & Medium land & Landrace & $\begin{array}{l}\text { Tarai Research Society, } \\
\text { Alipurduar, West Bengal }\end{array}$ \\
\hline 6. & Tulsibhog & $\begin{array}{l}\text { Photo-period sensitive, } \\
\text { long duration, tall, medium } \\
\text { slender grain, low yield } \\
\text { potential }(1.5-2.0 \text { t/ha) }\end{array}$ & $\begin{array}{l}\text { Northern part of West } \\
\text { Bengal }\end{array}$ & Medium or low land & Landrace & $\begin{array}{l}\text { Tarai Research Society, } \\
\text { Alipurduar, West Bengal }\end{array}$ \\
\hline 7. & Kalturey & $\begin{array}{l}\text { Photo-period sensitive, long } \\
\text { duration, tall, highly lodg- } \\
\text { ing susceptible, medium } \\
\text { slender grain, low yield } \\
\text { potential (1.5-2.0 t/ha) }\end{array}$ & Darjeeling Hills & Hill slope & Landrace & $\begin{array}{l}\text { Darjeeling KVK, West } \\
\text { Bengal }\end{array}$ \\
\hline 8. & Kataribhog & $\begin{array}{l}\text { Photo-period sensitive, } \\
\text { long duration, tall, slender } \\
\text { grain, low yield potential } \\
(1.5-2.0 \mathrm{t} / \mathrm{ha})\end{array}$ & $\begin{array}{l}\text { Northern part of West } \\
\text { Bengal }\end{array}$ & Medium or low land & Landrace & $\begin{array}{l}\text { Tarai Research Society, } \\
\text { Alipurduar, West Bengal }\end{array}$ \\
\hline 9. & Sitalkuchi-1(A1-1) & $\begin{array}{l}\text { Photo-period sensitive, } \\
\text { long duration, tall, slender } \\
\text { grain, low yield potential } \\
(1.5-2.0 \mathrm{t} / \mathrm{ha})\end{array}$ & $\begin{array}{l}\text { Sitalkuchi block, Cooch } \\
\text { Behar district, Northern } \\
\text { part of West Bengal }\end{array}$ & Medium or low land & Selection from Sitalkuchi-1 & $\begin{array}{l}\text { Sitalkuchi block, Cooch } \\
\text { Behar district, West Bengal }\end{array}$ \\
\hline 10. & Dubarikomal & $\begin{array}{l}\text { Photo-period sensitive, long } \\
\text { duration, tall, short-bold } \\
\text { grain, brown-red grain } \\
\text { husk, lodging suscepti- } \\
\text { ble, low yield potential } \\
(2.0-2.5 \mathrm{t} / \mathrm{ha})\end{array}$ & $\begin{array}{l}\text { Lower-eastern part of } \\
\text { Assam }\end{array}$ & Medium or low land & Landrace & $\begin{array}{l}\text { ICAR-CPCRI- Kahikuchi, } \\
\text { Kamrup, Assam }\end{array}$ \\
\hline 11. & Dharmaphou & $\begin{array}{l}\text { Photo-period sensitive, } \\
\text { long duration, tall, bold } \\
\text { grain, low yield potential } \\
(2.5-3.0 \mathrm{t} / \mathrm{ha})\end{array}$ & $\begin{array}{l}\text { Lower-eastern part of } \\
\text { Assam }\end{array}$ & Medium or low land & Landrace & $\begin{array}{l}\text { ICAR-CPCRI- Kahikuchi, } \\
\text { Kamrup, Assam }\end{array}$ \\
\hline 12. & Dudheswar & $\begin{array}{l}\text { Photo-period sensitive, } \\
\text { long duration, tall, short- } \\
\text { bold grain, good eating } \\
\text { quality, low yield potential } \\
(2.5-3.0 \mathrm{t} / \mathrm{ha})\end{array}$ & $\begin{array}{l}\text { Northern part of West } \\
\text { Bengal }\end{array}$ & Medium or low land & Landrace & $\begin{array}{l}\text { Tarai Research Society, } \\
\text { Alipurduar, West Bengal }\end{array}$ \\
\hline 13. & Dudhkalam & $\begin{array}{l}\text { Photo-period sensitive, } \\
\text { long duration, tall, short- } \\
\text { bold grain, good eating } \\
\text { quality, low yield potential } \\
(2.5-3.0 \mathrm{t} / \mathrm{ha})\end{array}$ & $\begin{array}{l}\text { Northern part of West } \\
\text { Bengal }\end{array}$ & Medium or low land & Landrace & $\begin{array}{l}\text { Tarai Research Society, } \\
\text { Alipurduar, West Bengal }\end{array}$ \\
\hline 14. & Rampha & $\begin{array}{l}\text { Photo-period sensitive, } \\
\text { long duration, tall, bold } \\
\text { grain, low yield potential } \\
(2.0-2.5 \mathrm{t} / \mathrm{ha})\end{array}$ & $\begin{array}{l}\text { Lower-eastern part of } \\
\text { Assam }\end{array}$ & Medium or low land & Landrace & $\begin{array}{l}\text { ICAR-CPCRI- Kahikuchi, } \\
\text { Kamrup, Assam }\end{array}$ \\
\hline 15. & Gobindabhog & $\begin{array}{l}\text { Photo-period sensitive, } \\
\text { long duration, tall, short- } \\
\text { bold grain, good eating } \\
\text { quality, low yield potential } \\
(3.0-3.5 \mathrm{t} / \mathrm{ha})\end{array}$ & $\begin{array}{l}\text { Lower western part of } \\
\text { West Bengal (Burdhawan } \\
\text { district) }\end{array}$ & Medium or low land & Landrace & $\begin{array}{l}\text { BCKV, Mohanpur, West } \\
\text { Bengal }\end{array}$ \\
\hline 16. & Konkanijoha & $\begin{array}{l}\text { Photo-period sensitive, long } \\
\text { duration, tall, short-bold } \\
\text { grain with black husk, low } \\
\text { yield potential }(2.0-2.5 \mathrm{t} / \mathrm{ha})\end{array}$ & $\begin{array}{l}\text { Lower-eastern part of } \\
\text { Assam }\end{array}$ & Medium or low land & Landrace & $\begin{array}{l}\text { ICAR-CPCRI- Kahikuchi, } \\
\text { Kamrup, Assam }\end{array}$ \\
\hline
\end{tabular}




\begin{tabular}{|c|c|c|c|c|c|c|}
\hline Sl no. & Name of the genotype & $\begin{array}{l}\text { Description of the } \\
\text { genotype }\end{array}$ & Origin & Ecology & Parentage & $\begin{array}{l}\text { Place of collection/ } \\
\text { source of the seed }\end{array}$ \\
\hline 17. & Kalokhasa & $\begin{array}{l}\text { Photo-period sensitive, } \\
\text { long duration, tall, short- } \\
\text { bold grain, good eating } \\
\text { quality, low yield potential } \\
(3.0-3.5 \mathrm{t} / \mathrm{ha})\end{array}$ & $\begin{array}{l}\text { Murshidabad district, West } \\
\text { Bengal }\end{array}$ & Medium or low land & Landrace & $\begin{array}{l}\text { Murshidabad district, West } \\
\text { Bengal }\end{array}$ \\
\hline 18. & Kabra & $\begin{array}{l}\text { Photo-period sensitive, } \\
\text { long duration, tall, bold } \\
\text { grain, low yield potential } \\
(2.0-2.5 \mathrm{t} / \mathrm{ha})\end{array}$ & $\begin{array}{l}\text { Lower-eastern part of } \\
\text { Assam }\end{array}$ & Medium or low land & Landrace & $\begin{array}{l}\text { ICAR-CPCRI- Kahikuchi, } \\
\text { Kamrup, Assam }\end{array}$ \\
\hline 19. & Radhunipagol & $\begin{array}{l}\text { Photo-period sensitive, } \\
\text { long duration, tall, lodging } \\
\text { susceptible, short-bold } \\
\text { grain, low yield potential } \\
(2.0-2.5 \mathrm{t} / \mathrm{ha})\end{array}$ & $\begin{array}{l}\text { Lower western part of West } \\
\text { Bengal }\end{array}$ & Medium or low land & Landrace & $\begin{array}{l}\text { BCKV, Mohanpur, West } \\
\text { Bengal }\end{array}$ \\
\hline 20. & Kalojeera & $\begin{array}{l}\text { Photo-period sensitive, long } \\
\text { duration, tall, lodging sus- } \\
\text { ceptible, short-bold grain } \\
\text { with black husk, low yield } \\
\text { potential }(1.5-2.0 \mathrm{t} / \mathrm{ha})\end{array}$ & $\begin{array}{l}\text { Lower western part of West } \\
\text { Bengal }\end{array}$ & Medium or low land & Landrace & $\begin{array}{l}\text { BCKV, Mohanpur, West } \\
\text { Bengal }\end{array}$ \\
\hline 21. & Kalonunia & $\begin{array}{l}\text { Photo-period sensitive, } \\
\text { long duration, tall, lodging } \\
\text { susceptible, medium slender } \\
\text { grain, low yield potential } \\
(1.5-2.0 \mathrm{t} / \mathrm{ha})\end{array}$ & $\begin{array}{l}\text { Northern part of West } \\
\text { Bengal }\end{array}$ & Medium or low land & Landrace & $\begin{array}{l}\text { Rice Repository, UBKV, } \\
\text { Cooch Behar }\end{array}$ \\
\hline 22. & Chakhao Sel-I & $\begin{array}{l}\text { Photo-period sensitive, long } \\
\text { duration, medium tall, bold } \\
\text { grain with black kernel, low } \\
\text { yield potential (1.5-2.0 t/ha) }\end{array}$ & Selection from Chakhao & Medium or low land & Landrace & $\begin{array}{l}\text { Rice Repository, UBKV, } \\
\text { Cooch Behar }\end{array}$ \\
\hline 23. & Chakhao Poiterin & $\begin{array}{l}\text { Photo-period sensitive, long } \\
\text { duration, medium tall, bold } \\
\text { grain with black kernel, low } \\
\text { yield potential (1.5-2.0 t/ha) }\end{array}$ & Manipur & Medium or low land & Landrace & Manipur \\
\hline 24. & Chapka Chakhao & $\begin{array}{l}\text { Photo-period sensitive, long } \\
\text { duration, medium tall, bold } \\
\text { grain with black kernel, low } \\
\text { yield potential }(1.5-2.0 \mathrm{t} / \mathrm{ha})\end{array}$ & Manipur & Medium or low land & Landrace & Manipur \\
\hline 25. & Chakhao Sel-2 & $\begin{array}{l}\text { Photo-period sensitive, long } \\
\text { duration, medium tall, bold } \\
\text { grain with black kernel, low } \\
\text { yield potential (1.5-2.0 t/ha) }\end{array}$ & Selection from Chakhao & Medium or low land & Landrace & $\begin{array}{l}\text { Rice Repository, UBKV, } \\
\text { Cooch Behar }\end{array}$ \\
\hline 26. & Chakhao Sel-3 & $\begin{array}{l}\text { Photo-period sensitive, long } \\
\text { duration, medium tall, bold } \\
\text { grain with black kernel, low } \\
\text { yield potential (1.5-2.0 t/ha) }\end{array}$ & Selection from Chakhao & Medium or low land & Landrace & $\begin{array}{l}\text { Rice Repository, UBKV, } \\
\text { Cooch Behar }\end{array}$ \\
\hline 27. & Chakhao Sampark & $\begin{array}{l}\text { Photo-period sensitive, long } \\
\text { duration, medium tall, bold } \\
\text { grain with black kernel, low } \\
\text { yield potential }(1.5-2.0 \mathrm{t} / \mathrm{ha})\end{array}$ & Manipur & Medium or low land & Landrace & Manipur \\
\hline 28. & KNS-2-1 & $\begin{array}{l}\text { Photo-period sensitive, } \\
\text { long duration, tall, lodging } \\
\text { susceptible, medium slender } \\
\text { grain, low yield potential } \\
(1.5-2.0 \mathrm{t} / \mathrm{ha})\end{array}$ & Selection from KaloNunia & Medium or low land & Landrace & $\begin{array}{l}\text { Rice Repository, UBKV, } \\
\text { Cooch Behar }\end{array}$ \\
\hline 29. & $\begin{array}{l}\text { KNS-3-1 (Uttar Sugandhi) } \\
\text { (IET 24616) }\end{array}$ & $\begin{array}{l}\text { Photo-period sensitive, } \\
\text { long duration, tall, lodging } \\
\text { susceptible, medium slender } \\
\text { grain, low yield potential } \\
(2.0-2.5 \mathrm{t} / \mathrm{ha})\end{array}$ & Selection from KaloNunia & Medium or low land & Landrace & $\begin{array}{l}\text { Rice Repository, UBKV, } \\
\text { Cooch Behar }\end{array}$ \\
\hline 30. & KNS-2-1-1 & $\begin{array}{l}\text { Photo-period sensitive, } \\
\text { long duration, tall, lodging } \\
\text { susceptible, medium slender } \\
\text { grain, low yield potential } \\
(2.0-2.5 \mathrm{t} / \mathrm{ha})\end{array}$ & Selection from KaloNunia & Medium or low land & Landrace & $\begin{array}{l}\text { Rice Repository, UBKV, } \\
\text { Cooch Behar }\end{array}$ \\
\hline 31. & KNS-2B-S1 & $\begin{array}{l}\text { Photo-period sensitive, } \\
\text { long duration, tall, lodging } \\
\text { susceptible, medium slender } \\
\text { grain, low yield potential } \\
(2.0-2.5 \mathrm{t} / \mathrm{ha})\end{array}$ & Selection from KaloNunia & Medium or low land & Landrace & $\begin{array}{l}\text { Rice Repository, UBKV, } \\
\text { Cooch Behar }\end{array}$ \\
\hline 32. & T4M-3-5 & $\begin{array}{l}\text { Photo-period insensitive, } \\
\text { medium duration, semi- } \\
\text { dwarf, lodging tolerant, } \\
\text { medium slender grain with } \\
\text { long awn, medium yield } \\
\text { potential }(3.5-4.0 \mathrm{t} / \mathrm{ha})\end{array}$ & Tulaipanji mutant & Medium and upland & Mutant of Tulaipanji & $\begin{array}{l}\text { Rice Repository, UBKV, } \\
\text { Cooch Behar }\end{array}$ \\
\hline
\end{tabular}




\begin{tabular}{|c|c|c|c|c|c|c|}
\hline Sl no. & Name of the genotype & $\begin{array}{l}\text { Description of the } \\
\text { genotype }\end{array}$ & Origin & Ecology & Parentage & $\begin{array}{l}\text { Place of collection/ } \\
\text { source of the seed }\end{array}$ \\
\hline 33. & TSP6-M3-4 & $\begin{array}{l}\text { Photo-period insensitive, } \\
\text { medium duration, semi- } \\
\text { dwarf, lodging tolerant, } \\
\text { medium slender grain with } \\
\text { long awn, medium yield } \\
\text { potential }(3.5-4.0 \mathrm{t} / \mathrm{ha})\end{array}$ & Tulaipanji mutant & Medium and upland & Mutant of Tulaipanji & $\begin{array}{l}\text { Rice Repository, UBKV, } \\
\text { Cooch Behar }\end{array}$ \\
\hline 34. & $\begin{array}{l}\text { TSPM-3-1 (TPUR-B-1-IET } \\
\text { 28104) }\end{array}$ & $\begin{array}{l}\text { Photo-period insensitive, } \\
\text { medium duration, semi- } \\
\text { dwarf, lodging tolerant, } \\
\text { medium slender grain with } \\
\text { long awn, medium yield } \\
\text { potential }(3.5-4.0 \text { t/ha) }\end{array}$ & Tulaipanji mutant & Medium and upland & Mutant of Tulaipanji & $\begin{array}{l}\text { Rice Repository, UBKV, } \\
\text { Cooch Behar }\end{array}$ \\
\hline 35. & T6M-3-3 & $\begin{array}{l}\text { Photo-period insensitive, } \\
\text { medium duration, semi- } \\
\text { dwarf, lodging tolerant, } \\
\text { medium slender grain with } \\
\text { long awn, medium yield } \\
\text { potential }(3.5-4.0 \text { t/ha) }\end{array}$ & Tulaipanji mutant & Medium and upland & Mutant of Tulaipanji & $\begin{array}{l}\text { Rice Repository, UBKV, } \\
\text { Cooch Behar }\end{array}$ \\
\hline
\end{tabular}

Table 2. Description of the selected traditionally important aromatic cultivars along with photo insensitive line developed from these aromatic cultivars.

Based on the nutritional parameters, the genotypes were clustered statistically. Resistant Starch (RS), Glycemic Index (GI), Non-Reducing Sugar (NRS) and Total Soluble Solids (TSS) were found to be contributing to the variability of the cultivars (Fig. 4A, Supplementary information 3a). Both the cluster plot and Principal Component Analysis divided the genotypes in four distinct clusters (Fig. 4B). The first cluster with genotypes like 'Kataribhog', 'Radhatilak', 'Radhunipagol', 'Dudhkalam, 'Kalokhasa,' 'Dubarikomal', 'Dharmaphou', 'Konkanijoha' etc. were found to be low in Glycemic index (GI) and high in Resistant Starch (RS). The second cluster were constituted by 'Rampha,' 'Tulaipanji,' 'Kalonunia, 'Dudheswar, 'Kalshipa, 'Kalojeera, 'KNS-2'-1', 'KNS-3'-1', 'T6M-3-3' have relatively high GI values. The third group constituted of the photo insensitive lines of 'Tulaipanji' like 'TSP6M3-4, 'T4M-3-5, 'TPUR-B-1(IET 28104)' along the black varieties like 'Chakhao sel-3', 'Chakhao sel-2', 'Chakhao Poiterin' and two popular genotypes 'Sadanunia' and 'Gobindabhog. All these genotypes exhibited high starch content. PCA of the variables were performed and Resistant Starch (RS), Total Soluble Sugar (TSS) and Glycemic Index (GI) were found to be significantly contributing in the grouping of the genotypes. The Strach content (STA), Glycemic Index (GI) were found in opposite dimension to Resistant Starch (RS) in PCoA biplot (Fig. 4C). Correlation analysis suggested that the RS and the GI are negatively correlated whereas Protein (PRO), Antioxidant activity (ANT) and Aroma (ARO) were found positively correlated in these genotypes (Fig. 4D).

Considering the high nutritional and ethno-medicinal values of local land races, the present study has special merits. Starch is the major contributor which determines the appearance of rice after cooking and its consistency ${ }^{40}$. The starch granules are aggregation of linear amylose chain and highly branched amylopectin fractions ${ }^{41}$. The proportion of amylose and amylopectin has profound effect on the physicochemical properties of rice like stickiness, water absorption, volume expansion, hardness and texture of rice ${ }^{40}$. The amylose content of Pusa Basmati was found to be less when compared with 'Gobindabhog' ${ }^{\text {'2 }}$. Along with good cooking quality, modern health-conscious consumers prefer rice with high medicinal value. Due to the lifestyle diseases like obesity, Type II diabetes mellitus, hypertension, etc. the popularity of rice is declining in many countries as white starch is considered to be one of the detrimental source of energy. Very few studies have been conducted for the estimation of health benefitting GI and RS of the Indian scented rice. Rice generally contains less than $3 \%$ of type 5 resistant $\operatorname{starch}^{43}$. Resistant starch produces zero calories on digestion, and offers health benefit for diabetic patients. Many of the previous work has also reported strong negative correlation of RS and GI in rice cultivars even after cooking ${ }^{44}$ where as high amylose content rice cultivars have been reported to have low GI ${ }^{24,45}$. GI of Pusa Basmati 1121 has been reported as $58.41 \%$ which has been shown to reduce by steaming of the paddy or by different cooking treatments ${ }^{46,47}$. As compared to basmati; the land races like, 'Kataribhog', 'A-1-1', 'Dubarikomal', 'KNS-2B-S1', 'TPUR-B-1 (IET 28104)' etc. demonstrates very good health parameters with low GI and high RS. Although black rice has been known to have high antioxidant activity but the local genotypes like 'Sadanunia', 'Rangakomal', 'Kalshipa' etc. has shown promising antioxidant activity which is comparable with black cultivars. Non-basmati aromatic cultivars from Bangladesh have been evaluated for their phenol and flavonoid content and have been proved to have good antioxidant potential ${ }^{48}$. Cultivars like 'Gopalbhog', 'Gobindabhog', 'Badshabhog' etc. has been found to have good nutritional value ${ }^{49}$. Altogether the results indicate that the local cultivars of these region have very good quality traits and can be popularized as healthy rice or may be utilized in breeding programs for quality enhancement.

Screening of the selected aromatic genotypes against rice blast disease. The sub-Himalayan Terai region is hot spot for blast disease and the meteorological data indicates that mid-September to late October is the most suitable time for the outbreak of the disease (Supplementary dataset 1). The AUDPC (Area Under Disease Progress Curve) indicates that cultivars like 'Sadanunia,' 'Tulsibhog', 'Chakhao Sampark, 'T4M-3-5' etc. exhibited extreme tolerance for leaf blast disease whereas 'Gobindabhog', 'Konkanijoha, 'Kalonunia', 'Rampha', 'KNS-2B-S1', 'KNS-3'-1' etc. were found to be highly susceptible to leaf blast under natural conditions (Table 6, Fig. 5A). From the PDI values it has been noticed that the disease progresses very rapidly in the month of 


\begin{tabular}{|c|c|c|c|c|c|c|c|c|}
\hline Primer ID & Chr no & Forward sequence & Reverse sequence & $\begin{array}{l}\text { Monomorphic/ } \\
\text { polymorphic }\end{array}$ & Allele no & $\begin{array}{l}\text { Amplicon } \\
\text { size (bp) }\end{array}$ & $\begin{array}{l}\text { Annealing } \\
\text { temp }\left({ }^{\circ} \mathrm{C}\right)\end{array}$ & \% Polymorphic \\
\hline RM 1 & 1 & $\begin{array}{l}\text { GCGAAAACACAATGC } \\
\text { AAAAA }\end{array}$ & $\begin{array}{l}\text { GCGTTGGTTGGACCT } \\
\text { GAC }\end{array}$ & Polymorphic & 1 & 100 & 51 & 20.00 \\
\hline RM 23 & 1 & $\begin{array}{l}\text { CATTGGAGTGGAGGC } \\
\text { TGG }\end{array}$ & $\begin{array}{l}\text { GTCAGGCTTCTGCCA } \\
\text { TTCTC }\end{array}$ & Polymorphic & 3 & $50-350$ & 55 & 15.24 \\
\hline RM 38 & 8 & $\begin{array}{l}\text { ACGAGCTCTCGATCA } \\
\text { GCCTA }\end{array}$ & $\begin{array}{l}\text { TCGGTCTCCATGTCC } \\
\text { CAC }\end{array}$ & Polymorphic & 1 & 900 & 59 & 22.86 \\
\hline RM 108 & 9 & $\begin{array}{l}\text { TCTCTTGCGCGCACA } \\
\text { CTGGCAC }\end{array}$ & $\begin{array}{l}\text { CGTGCACCACCACCA } \\
\text { CCACCAC }\end{array}$ & Polymorphic & 1 & 80 & 66 & 97.14 \\
\hline RM 114 & 3 & $\begin{array}{l}\text { CAGGGACGAATCGTC } \\
\text { GCCGGAG }\end{array}$ & $\begin{array}{l}\text { TTGGCCCCCTTGAGG } \\
\text { TTGTCGG }\end{array}$ & Polymorphic & 2 & $200-450$ & 66 & 80.00 \\
\hline RM 159 & 5 & $\begin{array}{l}\text { GGGGCACTGGCAAGG } \\
\text { GTGAAGG }\end{array}$ & $\begin{array}{l}\text { GCTTGTGCTTCTCTC } \\
\text { TCTCTCTCTCTCTC }\end{array}$ & Polymorphic & 4 & $150-650$ & 55 & 89.29 \\
\hline RM 165 & 1 & $\begin{array}{l}\text { CCGAACGCCTAGAAG } \\
\text { CGCGTCC }\end{array}$ & $\begin{array}{l}\text { CGGCGAGGTTTGCTA } \\
\text { ATGGCGG }\end{array}$ & Polymorphic & 2 & $200-300$ & 58 & 34.29 \\
\hline RM 169 & 5 & $\begin{array}{l}\text { TGGCTGGCTCCGTGG } \\
\text { GTAGCTG }\end{array}$ & $\begin{array}{l}\text { TCCCGTTGCCGTTCA } \\
\text { TCCCTCC }\end{array}$ & Polymorphic & 2 & $200-900$ & 62 & 62.86 \\
\hline RM 172 & 7 & $\begin{array}{l}\text { TGCAGCTGCGCCACA } \\
\text { GCCATAG }\end{array}$ & $\begin{array}{l}\text { CAACCACGACACCGC } \\
\text { CGTGTTG }\end{array}$ & Polymorphic & 1 & 180 & 51 & 40.00 \\
\hline RM 195 & 8 & $\begin{array}{l}\text { AGAAAGAGAGGCCGT } \\
\text { CGGCGGC }\end{array}$ & $\begin{array}{l}\text { GGGCTCACCCCCAAA } \\
\text { CCTGCAG }\end{array}$ & Polymorphic & 1 & 300 & 60 & 42.86 \\
\hline RM 250 & 2 & $\begin{array}{l}\text { GGTTCAAACCAAGCT } \\
\text { GATCA }\end{array}$ & $\begin{array}{l}\text { GATGAAGGCCTTCCA } \\
\text { CGCAG }\end{array}$ & Polymorphic & 3 & $200-500$ & 51 & 29.52 \\
\hline RM 256 & 8 & $\begin{array}{l}\text { GACAGGGAGTGATTG } \\
\text { AAGGC }\end{array}$ & $\begin{array}{l}\text { GTTGATTTCGCCAAG } \\
\text { GGC }\end{array}$ & Polymorphic & 1 & 65 & 59 & 82.86 \\
\hline RM 285 & 9 & $\begin{array}{l}\text { CTGTGGGCCCAATAT } \\
\text { GTCAC }\end{array}$ & $\begin{array}{l}\text { GGCGGTGACATGGAG } \\
\text { AAAG }\end{array}$ & Polymorphic & 2 & $150-200$ & 55 & 52.86 \\
\hline RM 288 & 9 & $\begin{array}{l}\text { CCGGTCAGTTCAAGC } \\
\text { TCTG }\end{array}$ & $\begin{array}{l}\text { ACGTACGGACGTGAC } \\
\text { GAC }\end{array}$ & Polymorphic & 1 & 170 & 62 & 51.43 \\
\hline RM 291 & 5 & $\begin{array}{l}\text { GTTGCACTACGTATT } \\
\text { CTGAG }\end{array}$ & $\begin{array}{l}\text { GATCCAGATAAATGA } \\
\text { GGCAC }\end{array}$ & Polymorphic & 1 & 200 & 58 & 60.00 \\
\hline RM 294 & 1 & $\begin{array}{l}\text { TTGGCCTAGTGCCTC } \\
\text { CAATC }\end{array}$ & $\begin{array}{l}\text { GAGGGTACAACTTAG } \\
\text { GACGCA }\end{array}$ & Polymorphic & 2 & $180-200$ & 62 & 75.71 \\
\hline RM 311 & 10 & $\begin{array}{l}\text { TGGTAGTATAGGTAC } \\
\text { TAAACAT } \\
\end{array}$ & $\begin{array}{l}\text { TCCTATACACATACA } \\
\text { AACATAC } \\
\end{array}$ & Polymorphic & 1 & 300 & 62 & 34.29 \\
\hline RM 314 & 6 & $\begin{array}{l}\text { CTAGCAGGAACTCCT } \\
\text { TTCAGG }\end{array}$ & $\begin{array}{l}\text { AACATTCCACACACA } \\
\text { CACGC }\end{array}$ & Polymorphic & 1 & 170 & 62 & 31.43 \\
\hline RM 321 & 9 & $\begin{array}{l}\text { CCAACACTGCCACTC } \\
\text { TGTTC }\end{array}$ & $\begin{array}{l}\text { GAGGATGGACACCTT } \\
\text { GATCG }\end{array}$ & Polymorphic & 1 & 200 & 62 & 48.57 \\
\hline RM 327 & 2 & $\begin{array}{l}\text { CTACTCCTCTGTCCC } \\
\text { TCCTCTC }\end{array}$ & $\begin{array}{l}\text { CCAGCTAGACACAAT } \\
\text { CGAGC }\end{array}$ & Polymorphic & 1 & 200 & 64 & 45.71 \\
\hline RM 332 & 11 & $\begin{array}{l}\text { GCGAAGGCGAAGGTG } \\
\text { AAG }\end{array}$ & $\begin{array}{l}\text { CATGAGTGATCTCAC } \\
\text { TCACCC }\end{array}$ & Polymorphic & 1 & 180 & 62 & 40.00 \\
\hline RM 342 & 8 & $\begin{array}{l}\text { CCATCCTCCTACTTC } \\
\text { AATGAAG }\end{array}$ & $\begin{array}{l}\text { ACTATGCAGTGGTGT } \\
\text { CACCC }\end{array}$ & Polymorphic & 1 & 180 & 62 & 31.43 \\
\hline RM 434 & 9 & $\begin{array}{l}\text { GCCTCATCССТCTAA } \\
\text { CCCTC }\end{array}$ & $\begin{array}{l}\text { CAAGAAAGATCAGTG } \\
\text { CGTGG }\end{array}$ & Polymorphic & 1 & 185 & 62 & 97.14 \\
\hline RM 460 & 9 & $\begin{array}{l}\text { TGATCGACAGCGTTC } \\
\text { TTGAC }\end{array}$ & $\begin{array}{l}\text { GCCTGGCCCACATAA } \\
\text { TTAAG }\end{array}$ & Polymorphic & 1 & 300 & 62 & 14.29 \\
\hline RM 469 & 6 & $\begin{array}{l}\text { AGCTGAACAAGCCCT } \\
\text { GAAAG }\end{array}$ & $\begin{array}{l}\text { GACTTGGGCAGTGTG } \\
\text { ACATG }\end{array}$ & Monomorphic & 1 & 85 & 62 & 100.00 \\
\hline RM 3134 & 3 & $\begin{array}{l}\text { GCAGGCACAAAAGCA } \\
\text { AAGAG }\end{array}$ & $\begin{array}{l}\text { AGGTGAAGGTGCATT } \\
\text { GTGTG }\end{array}$ & Polymorphic & 1 & 185 & 62 & 28.57 \\
\hline RM 3872 & 3 & $\begin{array}{l}\text { GGAAGAAAGGATCTA } \\
\text { TATCA }\end{array}$ & $\begin{array}{l}\text { TACGATTTGTTTAAG } \\
\text { TTCAA }\end{array}$ & Polymorphic & 1 & 150 & 62 & 31.43 \\
\hline RM 6250 & 4 & $\begin{array}{l}\text { AACCTACGTTACCCT } \\
\text { GCACG }\end{array}$ & $\begin{array}{l}\text { GGCTCATGAGTTTCA } \\
\text { GAGGC }\end{array}$ & Polymorphic & 1 & 180 & 52 & 22.86 \\
\hline RM 7376 & 12 & $\begin{array}{l}\text { TCACCGTCACCTCTT } \\
\text { AAGTC }\end{array}$ & $\begin{array}{l}\text { GGTGGTTGTGTTCTG } \\
\text { TTTGG }\end{array}$ & Polymorphic & 1 & 200 & 62 & 40.00 \\
\hline RM 10022 & 1 & $\begin{array}{l}\text { CCTCCATAGAGTAAG } \\
\text { GTTTGCATGG }\end{array}$ & $\begin{array}{l}\text { CCTCCTCCTCTGTCT } \\
\text { TTCTCTGC }\end{array}$ & Polymorphic & 2 & $200-400$ & 56 & 68.57 \\
\hline RM 16655 & 4 & $\begin{array}{l}\text { CCTTGGAAGCTGGAA } \\
\text { CTTCACC }\end{array}$ & $\begin{array}{l}\text { GGCTCTTAGGTTAGA } \\
\text { TCCCACACG }\end{array}$ & Polymorphic & 1 & 200 & 60 & 88.57 \\
\hline RM 23835 & 9 & $\begin{array}{l}\text { TTCCGCTGTTTCTCT } \\
\text { TCTTGTGC }\end{array}$ & $\begin{array}{l}\text { CTGGTTCTGCTGGTT } \\
\text { CTGTAGTTGG }\end{array}$ & Polymorphic & 1 & 200 & 58 & 54.29 \\
\hline ISSR1 & - & (GGC)5AT & & Polymorphic & 2 & $400-2000$ & 66 & 41.43 \\
\hline ISSR2 & - & (AAG)5GC & & Polymorphic & 3 & $150-2000$ & 48 & 31.43 \\
\hline ISSR3 & - & (AAG)5TG & & Polymorphic & 3 & $150-2500$ & 50 & 42.86 \\
\hline
\end{tabular}




\begin{tabular}{|c|c|c|c|c|c|c|c|c|}
\hline Primer ID & Chr no & Forward sequence & Reverse sequence & $\begin{array}{l}\text { Monomorphic/ } \\
\text { polymorphic }\end{array}$ & Allele no & $\begin{array}{l}\text { Amplicon } \\
\text { size (bp) }\end{array}$ & $\begin{array}{l}\text { Annealing } \\
\text { temp }\left({ }^{\circ} \mathrm{C}\right)\end{array}$ & \% Polymorphic \\
\hline ISSR4 & - & (AAG)5CC & & Polymorphic & 3 & $150-2500$ & 50 & 43.81 \\
\hline ISSR5 & - & (AGC)5CA & & Polymorphic & 2 & $150-1000$ & 62 & 54.29 \\
\hline ISSR6 & - & (AGC)5CG & & Polymorphic & 3 & $500-3500$ & 52 & 43.81 \\
\hline ISSR7 & - & (GGC)5TA & & Polymorphic & 5 & $150-3000$ & 66 & 51.43 \\
\hline ISSR8 & - & (AGC)5GA & & Polymorphic & 4 & $250-1500$ & 62 & 40.71 \\
\hline ISSR9 & - & (AAG)5CG & & Polymorphic & 3 & $400-2900$ & 52 & 36.19 \\
\hline ISSR10 & - & CCA(GTG)4 & & Polymorphic & 2 & $300-2000$ & 60 & 78.57 \\
\hline
\end{tabular}

Table 3. Detailed of the SSR and ISSR markers used in the study. The primers for the SSR and ISSR markers were designed following article reference nos. ${ }^{27,28}$.

September for all the genotypes (Fig. 5B). The distinctness of the tolerant versus susceptible cultivars can be observed during this period. In-vitro disease progression assay suggested that the susceptible cultivars develop symptoms after $24 \mathrm{~h}$ of inoculation whereas the tolerant cultivars did not develop symptoms even after $48 \mathrm{~h}$ of inoculation (Fig. 5C). Time lapse microscopy of susceptible cultivars showed spore germination and hyphae development within $24 \mathrm{~h}$ post inoculation in susceptible cultivars like 'Gobindabhog' and 'Konkanijoha' unlike tolerant cultivars 'Sadanunia,' 'T4M-3-5' etc. (Fig. 5D). Both in field experimets and in-vitro experiments represent discreet difference in disease severity among the selected genotypes.

Identification of important phenotypic characters related to blast disease. Phenotypic data like plant height (PLH), tiller number (TLN), upper, lower and middle leaf angles (ULA, MLA, LLA respectively) in selected rice genotypes and their impact on the disease parameters like lesion numbers in upper, middle and lower leaves (LUL, LML, LLL respectively), lesion size (LSZ), sporulation center (SPC) and AUDPC were calculated (Table 6). Principal Component analysis showed that the disease parameters like lesion number in upper middle and lower leaf (LUL, LML and LLL), lesion type (LST), sporulation center (SPC) and AUDPC were found to be significantly contributing to the clustering of the genotypes (Fig. 6A, Supplementary information $3 \mathrm{~b}$ ). Among the morphological traits only upper leaf angle (ULA) significantly contributed to the clustering of the genotypes. Cluster analysis based on these disease parameters have resulted in four distinguished clusters for these genotypes (Fig. 6B). Cluster I and III constitutes of the genotypes like 'Sadanunia, 'Chakhao Sampark', 'TSP6-M3-4', 'T4M-3-5' etc. which showed low AUDPC value $(<600)$ with a smaller number of disease lesions in leaves and considered highly tolerant to the blast disease whereas genotypes in cluster III exhibited relatively higher AUDPC and is considered to be less tolerant to the disease. Cluster IV represented the cultivars which has high AUDPC (>1000) with dense disease lesion in all leaves and is considered to be highly susceptible to the blast disease. PCA analysis has demonstrated that AUDPC and disease lesions on lower, upper and middle leaf are placed on the same dimension whereas the leaf angle parameters were found to be falling in the opposite dimension (Fig. 6C). Correlation and regression analysis suggested that upper and middle leaf angle exhibits a significant negative correlation with AUDPC (Table 7, Fig. 6D). Tiller numbers and plant height were found to have non-significant but positive correlation with occurrence of the disease. The correlation study between the nutritional and disease parameters were also performed. Aroma (ARO) and antioxidant activity (ANT) was found to be have significant but weak positive correlation with AUDPC whereas Non-Reducing Sugar (NRS) content was found to have very weak negative correlation with AUDPC (Supplementary information 4) suggesting that the nutritional traits and disease resistance are unrelated independent characters.

Assessment of pi genes with the disease. The selected genotypes were screened for the presence of twentythree well characterized rice blast resistant pi gene (Table 8). Almost all the landraces were found to possess number of pi gene. Genotypes like 'A-1-1' and 'Kataribhog' contain all the twenty-three pi genes (Supplementary information 5). Lowest number of pi genes was found in 'T6M-3-3' and 'T4M-3-5'. Pi 27t showed the maximum density in the population and was found in every genotype. Similarly, Pi5, Pizt, pib, Pikm and Pita/Pita2 was found in these landraces with high density (Fig. 7). Pik-h, pi-9, pi-1 were found with very low frequency in these genotypes. To address the association of the pi genes, present in these genotypes with occurrence of the disease Kendall's-tau association analysis was performed using R package. All the genotypes from the cluster one, two and some genotypes from cluster three with less than 800 AUDPC was considered as tolerant and genotypes falling in cluster three and four with more than 800 AUDPC were considered as susceptible. Six pi genes pi37, pizt, pikh, pi9, pita-pita2, and pik showed strong Kendal-tau b association $(<0.001)$ with blast tolerance in these genotypes (Fig. 7, Supplementary information 6). Pikp, pikm and pi-33 also exhibited significant correlation with blast tolerance, whereas fourteen out of twentythree markers were found to have no association with blast tolerance in these genotypes (Fig. 7).

Fitted model for prediction of susceptibility to blast disease based on morphological traits. Based on the results of Principal Component analysis, significantly contributing variables except AUDPC were chosen for development of a logistic regression model for prediction of disease susceptibility of a genotype based on its morphological data. For testing the goodness of fit of the model Hosmer and Lemeshow test was performed which was found non-significant ( $p$ value 0.694 ) for the model at $5 \%$ level of significance indicating that the model fits well as for any goodness of fit. 


\begin{tabular}{|c|c|c|c|c|c|c|c|}
\hline Markers & $\mathrm{H}$ & PIC & $\mathrm{E}$ & H. Av & MI & D & $\mathbf{R}$ \\
\hline RM1 & 0.345 & 0.285 & 0.222 & 0.009 & 0.002 & 0.955 & 0.444 \\
\hline RM23 & 0.277 & 0.239 & 0.500 & 0.002 & 0.001 & \begin{tabular}{|l|}
0.973 \\
\end{tabular} & 1.00 \\
\hline RM38 & 0.345 & 0.285 & 0.222 & 0.009 & 0.002 & 0.955 & 0.444 \\
\hline RM108 & 0.054 & 0.052 & 0.972 & 0.001 & 0.001 & 0.055 & \begin{tabular}{|l|}
0.055 \\
\end{tabular} \\
\hline RM114 & 0.313 & 0.264 & 1.611 & 0.004 & 0.007 & 0.353 & 0.777 \\
\hline RM159 & 0.208 & 0.186 & \begin{tabular}{|l|}
3.527 \\
\end{tabular} & 0.001 & 0.005 & 0.222 & 0.944 \\
\hline RM165 & 0.461 & 0.354 & 0.722 & 0.006 & 0.004 & 0.872 & 1.22 \\
\hline RM169 & 0.461 & 0.354 & 1.277 & 0.006 & 0.008 & 0.595 & 0.555 \\
\hline RM172 & 0.475 & 0.362 & 0.388 & 0.013 & 0.005 & 0.855 & \begin{tabular}{|l|l|}
0.777 \\
\end{tabular} \\
\hline RM195 & 0.493 & 0.371 & 0.444 & 0.013 & 0.006 & 0.809 & 0.888 \\
\hline RM250 & 0.431 & \begin{tabular}{|l|}
0.338 \\
\end{tabular} & \begin{tabular}{|l|l|} 
\\
\end{tabular} & 0.003 & \begin{tabular}{|l|}
0.003 \\
\end{tabular} & 0.902 & 0.777 \\
\hline RM256 & 0.313 & 0.264 & 0.805 & 0.008 & 0.007 & 0.355 & 0.388 \\
\hline RM285 & 0.496 & 0.373 & 1.083 & 0.006 & \begin{tabular}{|l|}
0.007 \\
\end{tabular} & 0.710 & 1.611 \\
\hline RM288 & 0.500 & 0.375 & 0.500 & 0.013 & 0.006 & 0.757 & 1.00 \\
\hline RM291 & 0.486 & 0.367 & 0.583 & 0.013 & \begin{tabular}{|l|}
0.007 \\
\end{tabular} & 0.666 & 0.833 \\
\hline RM294 & 0.360 & 0.295 & 1.527 & 0.005 & \begin{tabular}{|l|}
0.007 \\
\end{tabular} & \begin{tabular}{|l|l|}
0.419 \\
\end{tabular} & 0.944 \\
\hline RM311 & 0.444 & 0.345 & 0.333 & 0.012 & 0.004 & 0.895 & 0.666 \\
\hline RM314 & 0.424 & 0.334 & 0.305 & 0.011 & \begin{tabular}{|l|}
0.003 \\
\end{tabular} & 0.912 & 0.611 \\
\hline RM321 & 0.498 & 0.374 & 0.472 & 0.013 & 0.006 & 0.784 & 0.944 \\
\hline RM327 & 0.498 & 0.374 & 0.472 & 0.013 & 0.006 & 0.784 & 0.944 \\
\hline RM332 & 0.475 & 0.362 & \begin{tabular}{|l|}
0.388 \\
\end{tabular} & 0.013 & 0.005 & \begin{tabular}{|l|}
0.855 \\
\end{tabular} & 0.777 \\
\hline RM342 & 0.424 & 0.334 & 0.305 & 0.011 & 0.003 & 0.912 & 0.611 \\
\hline RM434 & 0.054 & 0.052 & 0.972 & 0.001 & \begin{tabular}{|l|}
0.001 \\
\end{tabular} & 0.055 & 0.055 \\
\hline RM460 & 0.277 & 0.239 & 0.166 & 0.007 & 0.001 & 0.976 & 0.333 \\
\hline RM469 & 0 & 0 & 1 & 0 & 0 & 0 & 0 \\
\hline RM3134 & 0.424 & 0.334 & \begin{tabular}{|l|}
0.305 \\
\end{tabular} & 0.011 & 0.003 & 0.912 & 0.611 \\
\hline RM3872 & 0.424 & 0.334 & 0.305 & 0.011 & 0.003 & 0.912 & 0.611 \\
\hline RM6250 & 0.345 & 0.285 & 0.222 & 0.009 & 0.002 & 0.955 & 0.444 \\
\hline RM7376 & 0.475 & 0.362 & 0.388 & 0.013 & 0.005 & 0.855 & 0.777 \\
\hline RM10022 & 0.424 & 0.334 & 1.388 & 0.005 & 0.008 & 0.520 & 0.777 \\
\hline RM16655 & 0.197 & 0.178 & 0.888 & 0.005 & 0.004 & 0.212 & 0.222 \\
\hline RM23835 & 0.493 & 0.371 & 0.555 & 0.013 & 0.007 & 0.698 & 0.888 \\
\hline ISSR1 & 0.481 & 0.365 & 0.805 & 0.006 & 0.005 & 0.841 & 1.611 \\
\hline ISSR2 & 0.431 & 0.338 & 0.944 & 0.003 & 0.003 & 0.902 & 1.888 \\
\hline ISSR3 & 0.489 & 0.369 & 1.277 & 0.004 & 0.005 & 0.820 & 2.111 \\
\hline ISSR4 & 0.489 & 0.369 & 1.277 & 0.004 & 0.005 & 0.820 & 1.888 \\
\hline ISSR5 & 0.498 & 0.374 & 1.055 & 0.006 & 0.007 & 0.724 & 1.888 \\
\hline ISSR6 & 0.491 & 0.370 & 1.305 & 0.004 & 0.005 & 0.812 & 1.055 \\
\hline ISSR7 & 0.499 & 0.374 & 2.555 & 0.002 & 0.007 & 0.740 & 3.222 \\
\hline ISSR8 & 0.486 & 0.367 & 1.666 & 0.003 & 0.005 & 0.888 & 3.333 \\
\hline ISSR9 & 0.456 & 0.352 & 1.055 & 0.004 & 0.004 & 0.878 & 2.000 \\
\hline ISSR10 & 0.360 & 0.295 & 1.527 & 0.005 & 0.007 & 0.419 & 0.944 \\
\hline
\end{tabular}

Table 4. Polymorphism information of the thirty-five genotypes generated using microsatellite markers. $H$ : heterozygosity index, PIC: polymorphic information content, $E$ : effective multiplex ratio, $H$ :. av arithmetic mean of $\mathrm{H}, M I$ : marker index, $D$ : discriminating power, $R$ : resolving power.

The fitted model is:

$$
\begin{aligned}
& P[\text { Blast }=1] \\
& \quad=\frac{1}{1+\exp (5.566+0.892 * L U L-0.759 * L M L-2.845 * L S T-0.144 * L L L+2.847 * S P C-0.329 * U L A)}
\end{aligned}
$$

The model predicts that one unit increase in upper leaf lesion (LUL) will increase the odds in favor of blast occurrence by 2.439 times (Table 9, Supplementary information 7). On the other hand, one unit decrease in upper leaf angle (ULA) will increase the odds in favor of blast occurrence by 0.720 times. Sporulation center (SPC) was found to be the most important parameter for the prediction of blast occurrence. The fitted model is highly accurate in the sense that based on significant phenotypic parameters considered for the purpose the 


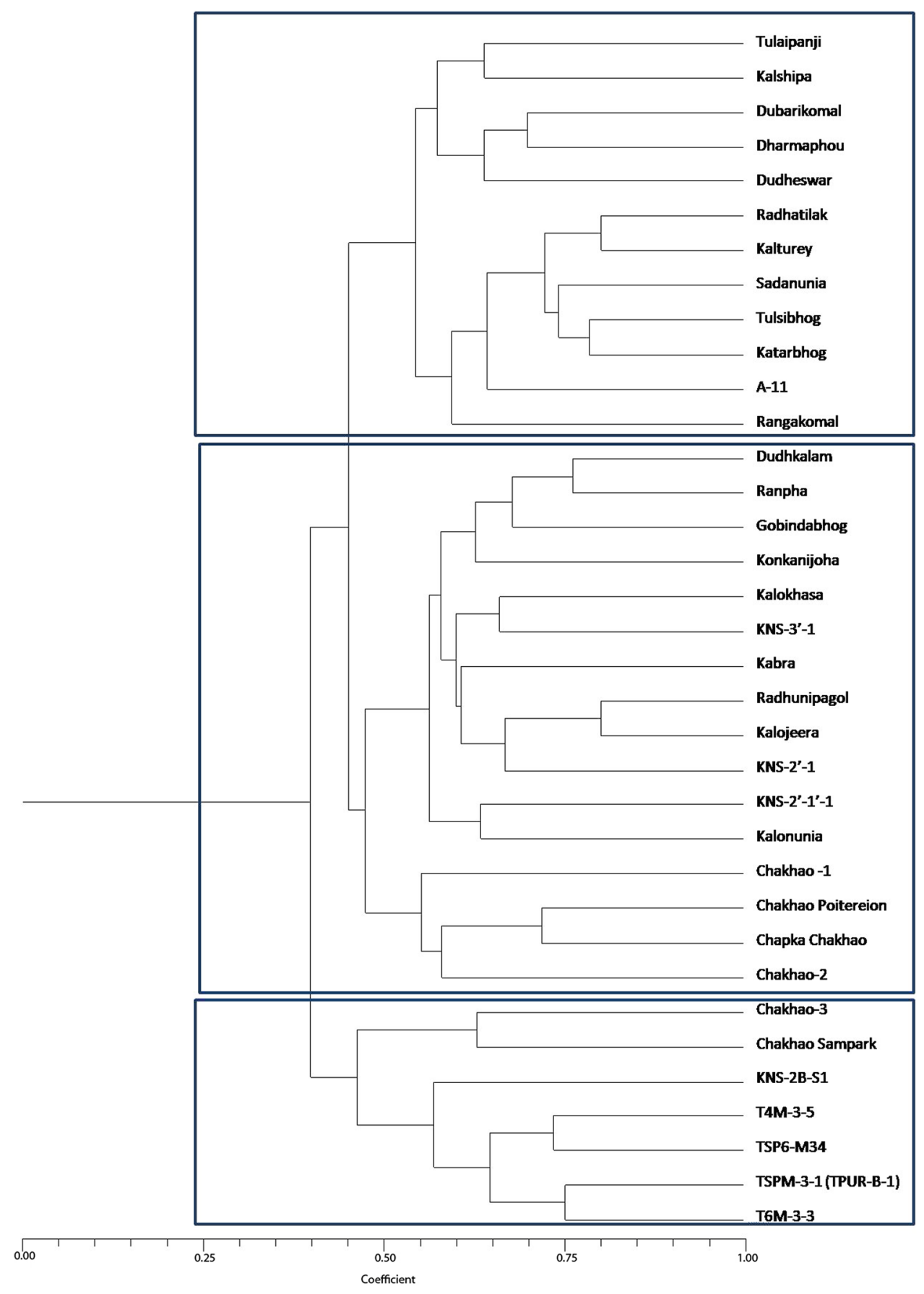

Figure 3. UPGMA based genetic clustering of thirty-five aromatic landraces determined by polymorphism obtained from SSR and ISSR markers using NTSYS-PC version $1.80^{30}$ (http://www.exetersoftware.com/cat/ntsys pc/ntsyspc.html).

overall correct classification rate among all the thirty-five genotypes is $75.7 \%$ whereas the correct classification rate is $86.7 \%$ within the susceptible genotypes under consideration (Supplementary information 7 ).

Rice blast caused by fungal pathogen Magnaporthe oryzae has been major constrain causing huge yield losses every year and considered as one of the most destructive disease of rice ${ }^{50-53}$. Indian subcontinent has faced seven severe epidemics due to the disease in last two decades ${ }^{53}$. Rice blast is influenced by several climatic conditions as relative humidity; temperature, light intensity etc. Based on the weather parameters several disease prediction models are available in different countries ${ }^{50,54}$, but disease prediction model based on morphological traits of the plant have been linked with blast disease is scarce. Morphological traits, like plant height, tiller number; leaf angle etc. has been reported to impact disease severity in many crops. Plant height and percentage of unfilled grain has been reported to be positively correlated to the severity of the disease ${ }^{55}$. Much earlier Mohanty et al. ${ }^{56}$ has reported a positive correlation of leaf angle, leaf pubescence, epicuticular wax, and quantity of deposition 


\begin{tabular}{|c|c|c|c|c|c|c|c|c|c|c|c|}
\hline Genotype & $\begin{array}{l}\text { Total Soluble } \\
\text { sugar }(\mathrm{g} / 100 \mathrm{~g})\end{array}$ & $\begin{array}{l}\text { Reducing sugar } \\
\text { (g/100 g) }\end{array}$ & $\begin{array}{l}\text { Non-reducing } \\
\text { sugar }(\mathrm{g} / 100 \mathrm{~g})\end{array}$ & Starch (\%) & Amylose (\%) & $\begin{array}{l}\text { Amylopectin } \\
(\%)\end{array}$ & $\begin{array}{l}\text { Resistant } \\
\text { starch (\%) }\end{array}$ & $\begin{array}{l}\text { Glycemic index } \\
\text { (\%) }\end{array}$ & Protein (\%) & $\begin{array}{l}\text { Antioxidant } \\
\text { activity }\left(\mathrm{IC}_{50}\right) \\
(\mu \mathrm{g} / \mathrm{ml})\end{array}$ & Aroma \\
\hline Tulaipanji & $53.96 \pm 1.71^{g-n}$ & $2.47 \pm 0.29^{\mathrm{a}-\mathrm{d}}$ & $0.77 \pm 0.15^{\mathrm{g}-\mathrm{k}}$ & $68.99 \pm 1.46^{\mathrm{i}-\mathrm{o}}$ & $20.43 \pm 1.88^{g-k}$ & $48.56 \pm 0.42^{f-g}$ & $0.87 \pm 0.09^{\mathrm{h}-\mathrm{m}}$ & $67.38 \pm 0.09^{b-d}$ & $8.30 \pm 0.10^{c-e}$ & $873.67 \pm 16.04^{0-q}$ & 3 \\
\hline Radhatilak & $80.58 \pm 3.07^{\mathrm{a}-\mathrm{d}}$ & $2.58 \pm 0.41^{\mathrm{a}-\mathrm{c}}$ & $0.85 \pm 0.12^{f-k}$ & $66.27 \pm 1.73^{k-p}$ & $23.43 \pm 0.94^{c-\mathrm{i}}$ & $42.83 \pm 0.79^{\mathrm{g}-\mathrm{i}}$ & $0.90 \pm 0.01^{\mathrm{h}-1}$ & $68.21 \pm 0.05^{\mathrm{b}, \mathrm{c}}$ & $8.91 \pm 0.10^{\mathrm{a}-\mathrm{c}}$ & $1231.75 \pm 15.87^{\mathrm{m}-\mathrm{o}}$ & 2 \\
\hline Kalshipa & $42.03 \pm 2.16^{\mathrm{m}, \mathrm{n}}$ & $2.46 \pm 0.55^{\mathrm{a}-\mathrm{d}}$ & $0.95 \pm 0.14^{e^{-j}}$ & $83.21 \pm 0.09^{\mathrm{a}, \mathrm{b}}$ & $26.93 \pm 1.17^{7^{-e}-\mathrm{e}}$ & $56.27 \pm 1.27^{\mathrm{d}-\mathrm{f}}$ & $0.92 \pm 0.20^{\mathrm{g}-\mathrm{k}}$ & $66.70 \pm 0.02^{\mathrm{b} \text {-e }}$ & $6.10 \pm 0.03^{\mathrm{h}-\mathrm{k}}$ & $314.72 \pm 65.23^{\mathrm{s}}$ & 1 \\
\hline Rongakomal & $88.24 \pm 1.37^{\mathrm{a}}$ & $2.40 \pm 0.21^{\mathrm{b}-\mathrm{d}}$ & $1.35 \pm 0.16^{\mathrm{b}-\mathrm{e}}$ & $83.17 \pm 0.69^{\mathrm{a}, \mathrm{b}}$ & $23.10 \pm 0.94^{\mathrm{d}-\mathrm{i}}$ & $60.07 \pm 0.24^{\mathrm{a}-\mathrm{d}}$ & $0.73 \pm 0.05^{\mathrm{h}-\mathrm{m}}$ & $64.67 \pm 0.03^{c-h}$ & $5.39 \pm 0.10^{\mathrm{k}-\mathrm{n}}$ & $408.76 \pm 22.64^{\mathrm{s}}$ & 2 \\
\hline Sadanunia & $45.02 \pm 0.91^{\mathrm{j}-\mathrm{n}}$ & $1.96 \pm 0.01^{c-e}$ & $1.04 \pm 0.23^{\mathrm{chh}}$ & $85.45 \pm 1.22^{\mathrm{a}}$ & $19.43 \pm 1.88^{\mathrm{h}-\mathrm{l}}$ & $66.01 \pm 0.65^{\mathrm{a}, \mathrm{b}}$ & $0.77 \pm 0.09^{\mathrm{h}-\mathrm{m}}$ & $60.49 \pm 0.06^{i-j}$ & $5.82 \pm 0.14^{i-1}$ & $338.79 \pm 34.85^{\mathrm{s}}$ & 2 \\
\hline Tulsibhog & $37.44 \pm 3.42^{\mathrm{n}}$ & $1.57 \pm 0.20^{e-g}$ & $0.76 \pm 0.24^{g-k}$ & $82.29 \pm 0.13^{\mathrm{a}-\mathrm{c}}$ & $29.10 \pm 2.82^{\mathrm{a}, \mathrm{b}}$ & $53.18 \pm 2.68^{e-f}$ & $0.97 \pm 0.05^{f-j}$ & $65.02 \pm 2.00^{\mathrm{b}-\mathrm{h}}$ & $8.65 \pm 0.18^{\mathrm{b}-\mathrm{d}}$ & $2028.44 \pm 42.14^{g-j}$ & 2 \\
\hline Kalturey & $43.16 \pm 2.62^{k-n}$ & $1.10 \pm 0.34^{f-j}$ & $0.69 \pm 0.02^{g-k}$ & $63.60 \pm 1.76^{\mathrm{m}-\mathrm{p}}$ & $9.93 \pm 0.23^{\mathrm{n}, \mathrm{o}}$ & $53.66 \pm 1.99^{\mathrm{clf}}$ & $0.93 \pm 0.03^{g-k}$ & $63.70 \pm 0.22^{\mathrm{b}-\mathrm{i}}$ & $3.86 \pm 0.25^{9}$ & $594.64 \pm 10.88^{q-s}$ & 3 \\
\hline Kataribhog & $64.77 \pm 1.48^{c-j}$ & $1.59 \pm 0.32^{e-j}$ & $0.77 \pm 0.17^{8-k}$ & $54.57 \pm 3.59^{q, r}$ & $20.43 \pm 5.65^{-k-k}$ & $34.13 \pm 9.25^{i-k}$ & $2.25 \pm 0.01^{\mathrm{a}}$ & $45.72 \pm 0.79^{\mathrm{m}}$ & $6.43 \pm 0.07^{\mathrm{h}, \mathrm{i}}$ & $1583.68 \pm 53.52^{k-m}$ & 2 \\
\hline A-1-1 & $77.43 \pm 2.50^{a-e}$ & $1.72 \pm 0.72^{\mathrm{d}-\mathrm{f}}$ & $0.66 \pm 0.13^{\mathrm{g}-\mathrm{k}}$ & $66.88 \pm 1.29^{-\mathrm{p}}$ & $13.26 \pm 2.59^{\mathrm{m}, \mathrm{n}}$ & $53.61 \pm 3.89^{c-f}$ & $1.79 \pm 0.01^{a-d}$ & $58.67 \pm 0.27^{j, k}$ & $9.34 \pm 0.14^{\mathrm{a}, \mathrm{b}}$ & $1065.78 \pm 54.27^{\mathrm{opp}}$ & 1 \\
\hline Dubarikomal & $63.65 \pm 2.39^{c-1}$ & $0.94 \pm 0.71^{f-j}$ & $0.89 \pm 0.09^{f-k}$ & $74.76 \pm 1.78^{c-j}$ & $7.60 \pm 0.70^{\circ}$ & $67.16 \pm 2.49^{\mathrm{a}}$ & $1.91 \pm 0.02^{2-c}$ & $54.77 \pm 0.07^{1}$ & $9.19 \pm 0.07^{\mathrm{a}, \mathrm{b}}$ & $1535.27 \pm 73.37^{\mathrm{k}-\mathrm{m}}$ & 2 \\
\hline Darmaphou & $62.52 \pm 1.48^{\mathrm{d}-\mathrm{n}}$ & $3.21 \pm 0.28^{\mathrm{a}}$ & $0.99 \pm 0.20^{\mathrm{d}-\mathrm{y}}$ & $70.22 \pm 1.48^{\mathrm{h}-\mathrm{o}}$ & $27.93 \pm 1.64^{\mathrm{a}-\mathrm{d}}$ & $42.28 \pm 0.16^{\mathrm{g}-\mathrm{i}}$ & $1.82 \pm 0.01^{\mathrm{a}-\mathrm{d}}$ & $62.70 \pm 0.17^{f-i}$ & $9.47 \pm 0.03^{\mathrm{a}}$ & $2216.74 \pm 91.96^{\mathrm{f}-\mathrm{h}}$ & 1 \\
\hline Dudheswar & $69.45 \pm 2.62^{\mathrm{a}-\mathrm{i}}$ & $3.10 \pm 1.05^{\mathrm{a}, \mathrm{b}}$ & $0.79 \pm 0.22 \mathrm{~g}^{\mathrm{k}}$ & $79.44 \pm 2.45^{\mathrm{a}-\mathrm{f}}$ & $13.27 \pm 1.17^{\mathrm{m}, \mathrm{n}}$ & $66.16 \pm 1.27^{\mathrm{a}, \mathrm{b}}$ & $1.44 \pm 0.01^{\mathrm{d}-\mathrm{f}}$ & $63.66 \pm 0.08^{\mathrm{b}-\mathrm{i}}$ & $5.87 \pm 0.14^{\mathrm{i}, 1}$ & $3241.72 \pm 39.58^{\mathrm{a}}$ & 1 \\
\hline Dudhkalam & $68.41 \pm 2.96^{-a j}$ & $1.50 \pm 0.55^{\mathrm{e}-\mathrm{g}}$ & $0.49 \pm 0.12^{\mathrm{j}, \mathrm{k}}$ & $49.94 \pm 5.91^{\mathrm{r}, \mathrm{s}}$ & $21.93 \pm 1.17^{e-1}$ & $28.01 \pm 4.73^{\mathrm{k}}$ & $1.39 \pm 0.03^{\mathrm{d}-\mathrm{g}}$ & $63.40 \pm 0.08^{\mathrm{b}-\mathrm{i}}$ & $7.68 \pm 0.10^{\mathrm{e}, \mathrm{f}}$ & $2489.88 \pm 21.02^{\mathrm{d}-\mathrm{f}}$ & 1 \\
\hline Ranpha & $55.42 \pm 0.56^{\mathrm{f}-\mathrm{n}}$ & $1.16 \pm 0.05^{e-\mathrm{i}}$ & $0.51 \pm 0.11^{\mathrm{i}-\mathrm{k}}$ & $82.93 \pm 7.07^{\mathrm{a}, \mathrm{b}}$ & $25.10 \pm 1.88^{\mathrm{b}-\mathrm{g}}$ & $57.82 \pm 5.18^{\mathrm{a}-\mathrm{f}}$ & $1.16 \pm 0.03^{\mathrm{e}-\mathrm{h}}$ & $65.33 \pm 0.03^{\mathrm{b}-\mathrm{h}}$ & $4.11 \pm 0.10^{\mathrm{q}, \mathrm{p}}$ & $2979.73 \pm 29.25^{\mathrm{a}-\mathrm{c}}$ & 3 \\
\hline Gobindabhog & $53.65 \pm 4.67^{\mathrm{j}-\mathrm{n}}$ & $0.87 \pm 0.30^{f-j}$ & $0.60 \pm 0.11^{\mathrm{h}-\mathrm{k}}$ & $73.39 \pm 5.68^{\mathrm{d}-\mathrm{m}}$ & $7.43 \pm 4.71^{\circ}$ & $65.95 \pm 0.96^{\mathrm{a}, \mathrm{b}}$ & $1.05 \pm 0.06^{-\mathrm{e}}$ & $66.14 \pm 0.11^{\mathrm{b}-\mathrm{f}}$ & $6.77 \pm 0.10^{\mathrm{gh}}$ & $1152.79 \pm 45.11^{\mathrm{n}-\mathrm{p}}$ & 3 \\
\hline Konkanijoha & $83.97 \pm 0.79^{\mathrm{a}-\mathrm{c}}$ & $0.91 \pm 0.23^{\mathrm{f}-\mathrm{j}}$ & $0.44 \pm 0.20^{\mathrm{k}}$ & $45.86 \pm 4.40^{\mathrm{s}}$ & $14.77 \pm 1.41^{1-\mathrm{m}}$ & $31.09 \pm 5.81^{j-k}$ & $0.97 \pm 0.04^{\mathrm{f}-\mathrm{j}}$ & $65.08 \pm 0.13^{\mathrm{b}-\mathrm{h}}$ & $9.29 \pm 0.22^{\mathrm{a}, \mathrm{b}}$ & $1859.23 \pm 20.60^{\mathrm{i}-\mathrm{k}}$ & 3 \\
\hline Kalokhasa & $67.03 \pm 4.67^{\mathrm{b}-\mathrm{i}}$ & $0.49 \pm 0.08^{\mathrm{i}, \mathrm{j}}$ & $0.41 \pm 0.02^{\mathrm{k}}$ & $60.06 \pm 2.27^{p, q}$ & $24.93 \pm 2.59^{\mathrm{b}-\mathrm{h}}$ & $35.12 \pm 4.86^{\mathrm{i}-\mathrm{k}}$ & $1.40 \pm 0.04^{\mathrm{d}-\mathrm{g}}$ & $62.25 \pm 0.01^{g-j}$ & $8.63 \pm 0.14^{\mathrm{b}-\mathrm{d}}$ & $1740.15 \pm 39.52^{j-1}$ & 1 \\
\hline Kabra & $64.21 \pm 5.24^{c-k}$ & $0.80 \pm 0.14^{8-j}$ & $0.60 \pm 0.14^{\mathrm{h}-\mathrm{k}}$ & $76.53 \pm 2.38^{\mathrm{b}-\mathrm{i}}$ & $28.77 \pm 0.47^{\mathrm{a}-\mathrm{c}}$ & $47.76 \pm 2.85^{\text {f-h }}$ & $1.14 \pm 0.02^{\mathrm{e}-\mathrm{h}}$ & $66.45 \pm 0.06^{\mathrm{b}-\mathrm{f}}$ & $4.75 \pm 0.07^{1 \mathrm{pp}}$ & $510.16 \pm 67.34^{\mathrm{ts}}$ & 3 \\
\hline Radhunipagol & $57.52 \pm 11.51^{e-n}$ & $0.73 \pm 0.17^{\mathrm{g}-\mathrm{j}}$ & $0.64 \pm 0.01^{\mathrm{g}-\mathrm{k}}$ & $78.49 \pm 4.96^{\mathrm{a}-\mathrm{g}}$ & $23.10 \pm 1.41^{\mathrm{g}-\mathrm{i}}$ & $55.38 \pm 6.37^{c-f}$ & $1.46 \pm 0.02^{\mathrm{ce}}$ & $58.86 \pm 0.07^{j-k}$ & $7.71 \pm 0.07^{\mathrm{e}, \mathrm{f}}$ & $1875.15 \pm 8.13^{\mathrm{h}-\mathrm{k}}$ & 3 \\
\hline Kalojeera & $61.87 \pm 1.93^{\mathrm{d}-\mathrm{n}}$ & $0.99 \pm 0.16^{f-j}$ & $0.75 \pm 0.12^{\mathrm{g}-\mathrm{k}}$ & $80.96 \pm 0.30^{\mathrm{a}-\mathrm{d}}$ & $19.10 \pm 0.47^{\mathrm{i}-1}$ & $61.86 \pm 0.17^{\mathrm{a}-\mathrm{d}}$ & $0.60 \pm 0.07^{\mathrm{i}-\mathrm{m}}$ & $74.36 \pm 0.13^{\mathrm{a}}$ & $5.74 \pm 0.25^{\mathrm{i}, 1}$ & $1777.82 \pm 40.00^{j-1}$ & 3 \\
\hline Kalonunia & $50.74 \pm 3.99^{\mathrm{h}-\mathrm{m}}$ & $1.02 \pm 0.28^{f-j}$ & $0.59 \pm 0.02^{\mathrm{h}-\mathrm{k}}$ & $72.49 \pm 1.94^{\mathrm{e}-\mathrm{m}}$ & $22.77 \pm 3.29^{\mathrm{d}-\mathrm{i}}$ & $49.71 \pm 5.24^{\mathrm{e}-\mathrm{g}}$ & $0.75 \pm 0.01^{\mathrm{h}-\mathrm{m}}$ & $66.85 \pm 0.13^{\text {b-e }}$ & $7.15 \pm 0.14^{\mathrm{fgg}}$ & $2147.88 \pm 14.89^{f-i}$ & 3 \\
\hline Chakhao sel-1 & $86.47 \pm 1.59^{\mathrm{a}, \mathrm{b}}$ & $0.87 \pm 0.57^{f-j}$ & $1.42 \pm 0.36^{\mathrm{b}-\mathrm{d}}$ & $76.55 \pm 2.08^{\mathrm{d}-\mathrm{i}}$ & $19.10 \pm 4.71^{\mathrm{i}-1}$ & $57.45 \pm 6.80^{\mathrm{a}-\mathrm{f}}$ & $1.65 \pm 0.01^{\mathrm{b}-\mathrm{d}}$ & $61.55 \pm 0.19^{\mathrm{h}-j}$ & $5.41 \pm 0.21^{\mathrm{j}-\mathrm{m}}$ & $2679.21 \pm 21.17^{\mathrm{cdd}}$ & 1 \\
\hline $\begin{array}{l}\text { Chakhao } \\
\text { Poiterein }\end{array}$ & $59.37 \pm 6.61^{e-m}$ & $0.77 \pm 0.05^{g-j}$ & $1.47 \pm 0.57^{\mathrm{b}, \mathrm{c}}$ & $63.39 \pm 11.80^{\mathrm{op}}$ & $7.77 \pm 2.82^{\circ}$ & $55.62 \pm 14.62^{\mathrm{c}-\mathrm{f}}$ & $0.40 \pm 0.04^{\mathrm{m}}$ & $60.81 \pm 0.12^{\mathrm{ij}}$ & $4.88 \pm 0.10^{\mathrm{m}-\mathrm{o}}$ & $596.99 \pm 68.78^{q-5}$ & 3 \\
\hline $\begin{array}{l}\text { Chapka } \\
\text { Chakhao }\end{array}$ & $75.74 \pm 1.02^{\mathrm{a}-\mathrm{f}}$ & $0.44 \pm 0.26^{\mathrm{i}, \mathrm{j}}$ & $1.51 \pm 0.09^{\mathrm{b}, \mathrm{c}}$ & $79.34 \pm 1.80^{\mathrm{a}-\mathrm{g}}$ & $19.93 \pm 0.70^{\mathrm{g}-1}$ & $59.40 \pm 2.51^{1-e}$ & $0.82 \pm 0.01^{\mathrm{h}-\mathrm{n}}$ & $64.94 \pm 0.12^{\mathrm{b}-\mathrm{h}}$ & $4.60 \pm 0.07^{\mathrm{m}-\mathrm{p}}$ & $823.74 \pm 103.55^{\mathrm{p}-\mathrm{r}}$ & 1 \\
\hline Chakhao-2 & $74.53 \pm 2.73^{\mathrm{a}-\mathrm{h}}$ & $0.41 \pm 0.02^{\mathrm{i}, \mathrm{j}}$ & $1.32 \pm 0.12^{\mathrm{c}-\mathrm{f}}$ & $65.94 \pm 0.20^{\mathrm{l}-\mathrm{b}}$ & $11.27 \pm 0.23^{\mathrm{m}-\mathrm{o}}$ & $54.67 \pm 0.44^{c-f}$ & $0.47 \pm 0.02^{\mathrm{k}-\mathrm{m}}$ & $64.90 \pm 0.23^{\mathrm{b}-\mathrm{h}}$ & $4.90 \pm 0.21^{1-o}$ & $386.43 \pm 76.83^{\mathrm{s}}$ & 1 \\
\hline Chakhao-3 & $58.00 \pm 1.71^{\mathrm{e}-\mathrm{n}}$ & $0.28 \pm 0.08^{j}$ & $1.12 \pm 0.25^{c-g}$ & $71.27 \pm 1.11^{\mathrm{g}-\mathrm{n}}$ & $7.93 \pm 0.70^{\circ}$ & $63.33 \pm 1.81^{\mathrm{a}-\mathrm{c}}$ & $0.47 \pm 0.08^{\mathrm{k}-\mathrm{m}}$ & $63.74 \pm 0.13^{\mathrm{b}-\mathrm{i}}$ & $5.23 \pm 0.10^{\mathrm{k}-\mathrm{n}}$ & $558.58 \pm 33.75^{9, x, s}$ & 1 \\
\hline $\begin{array}{l}\text { Chakhao } \\
\text { Sampark }\end{array}$ & $88.89 \pm 1.14^{\mathrm{e}-\mathrm{n}}$ & $0.31 \pm 0.04^{i j}$ & $1.39 \pm 0.24^{\mathrm{b}-\mathrm{e}}$ & $82.78 \pm 2.8^{7^{\mathrm{a}, \mathrm{b}}}$ & $24.93 \pm 4.00^{b-y}$ & $62.00 \pm 1.71^{-\mathrm{a} f}$ & $2.22 \pm 0.03^{\mathrm{d}-\mathrm{g}}$ & $59.55 \pm 0.11^{\mathrm{b}-\mathrm{f}}$ & $9.21 \pm 0.11^{\mathrm{d}, e}$ & $411.81 \pm 3.38^{\mathrm{op} p}$ & 1 \\
\hline KNS-2'-1 & $56.55 \pm 1.02^{e-n}$ & $1.40 \pm 0.09^{\mathrm{e}-\mathrm{h}}$ & $0.69 \pm 0.08^{g-k}$ & $78.50 \pm 1.08^{\mathrm{a}-\mathrm{g}}$ & $21.10 \pm 1.41^{\mathrm{f}-\mathrm{j}}$ & $57.40 \pm 2.50^{\mathrm{a}-\mathrm{f}}$ & $0.65 \pm 0.01^{\mathrm{i}-\mathrm{m}}$ & $74.73 \pm 0.22^{\mathrm{a}}$ & $7.38 \pm 0.10^{\mathrm{fgg}}$ & $3131.05 \pm 91.85^{\mathrm{a}, \mathrm{b}}$ & 2 \\
\hline $\begin{array}{l}\text { KNS-3'-1 } \\
\text { Uttar Sugandhi } \\
\text { (IET 24,616) }\end{array}$ & $54.37 \pm 5.01^{f^{-n}}$ & $0.95 \pm 0.22^{f-j}$ & $0.86 \pm 0.22^{f-k}$ & $74.12 \pm 4.40^{\mathrm{g}-\mathrm{k}}$ & $19.93 \pm 1.17^{-1}$ & $54.19 \pm 5.58^{\mathrm{c-f}}$ & $0.55 \pm 0.01^{\mathrm{i}-\mathrm{m}}$ & $72.80 \pm 0.05^{\mathrm{a}}$ & $6.13 \pm 0.28^{\mathrm{h}-j}$ & $2829.75 \pm 66.25^{\mathrm{b}-\mathrm{d}}$ & 2 \\
\hline KNS-2-1-1 & $58.89 \pm 4.33^{\mathrm{e}^{-\mathrm{m}}}$ & $0.77 \pm 0.09^{g-j}$ & $0.69 \pm 0.12^{g-k}$ & $80.52 \pm 4.17^{\mathrm{a}-\mathrm{e}}$ & $31.27 \pm 3.06^{\mathrm{a}}$ & $49.25 \pm 1.10^{e-g}$ & $2.11 \pm 0.01^{\mathrm{a}, \mathrm{b}}$ & $67.59 \pm 0.02^{b, c}$ & $5.44 \pm 0.10^{j-m}$ & $2281.55 \pm 12.51^{\mathrm{e}-\mathrm{g}}$ & 1 \\
\hline KNS-2B-S1 & $85.66 \pm 1.59^{\mathrm{a}, \mathrm{b}}$ & $0.59 \pm 0.13^{\mathrm{h}-\mathrm{j}}$ & $1.80 \pm 0.07^{\mathrm{a}, \mathrm{b}}$ & $77.40 \pm 0.27^{\mathrm{d}-\mathrm{h}}$ & $22.27 \pm 0.70^{e-i}$ & $55.13 \pm 0.42^{c-f}$ & $1.99 \pm 0.01^{\mathrm{a}, \mathrm{b}}$ & $55.08 \pm 0.14^{1}$ & $4.80 \pm 0.07^{1-p}$ & $1455.50 \pm 12.44^{1-\mathrm{n}}$ & 2 \\
\hline T4M-3-5 & $47.92 \pm 0.67^{\mathrm{i}-\mathrm{n}}$ & $0.35 \pm 0.07^{\mathrm{i}^{\mathrm{i}}}$ & $2.02 \pm 0.09^{\mathrm{a}}$ & $77.14 \pm 1.43^{\mathrm{d}-\mathrm{h}}$ & $16.27 \pm 0.23^{j-\mathrm{n}}$ & $60.87 \pm 1.20^{a-d}$ & $0.64 \pm 0.01^{\mathrm{i}-\mathrm{m}}$ & $66.03 \pm 0.16^{6-g}$ & $8.93 \pm 0.21^{a-c}$ & $1572.50 \pm 103.23^{\mathrm{k}-\mathrm{m}}$ & 2 \\
\hline TSP6-M3-4 & $59.29 \pm 5.35^{-\mathrm{m}}$ & $0.61 \pm 0.02^{\mathrm{h}-j}$ & $1.81 \pm 0.24^{\mathrm{a}, \mathrm{b}}$ & $72.03 \pm 1.34^{f-m}$ & $15.27 \pm 0.23^{k-n}$ & $56.75 \pm 1.58^{e-f}$ & $0.42 \pm 0.03^{1, \mathrm{~m}}$ & $68.54 \pm 0.20^{\mathrm{b}}$ & $5.23 \pm 0.18^{1-\mathrm{m}}$ & $1145.20 \pm 8.06^{\mathrm{n}-\mathrm{p}}$ & 1 \\
\hline $\begin{array}{l}\text { TSPM-3-1 } \\
\text { TPUR-B-1(IET } \\
\text { 28,104) }\end{array}$ & $61.79 \pm 0.67^{\mathrm{d}-\mathrm{n}}$ & $0.60 \pm 0.02^{\mathrm{h}-j}$ & $0.87 \pm 0.48^{f-k}$ & $73.68 \pm 0.71^{d-1}$ & $21.10 \pm 1.41^{\mathrm{f}_{-j}}$ & $52.58 \pm 2.13^{\mathrm{d}-\mathrm{f}}$ & $0.49 \pm 0.11^{k-m}$ & $55.63 \pm 0.03^{k, 1}$ & $5.77 \pm 0.14^{i-1}$ & $1605.10 \pm 16.68^{\mathrm{k}, 1}$ & 2 \\
\hline T6M-3-3 & $42.68 \pm 3.07^{1-\mathrm{n}}$ & $0.45 \pm 0.13^{\mathrm{i}, \mathrm{j}}$ & $0.70 \pm 0.31^{\mathrm{g}-\mathrm{k}}$ & $65.47 \pm 0.78^{\mathrm{m}-\mathrm{p}}$ & $26.60 \pm 1.64^{\mathrm{a}-\mathrm{f}}$ & $38.86 \pm 2.43^{\mathrm{h}-j}$ & $0.57 \pm 0.07^{\mathrm{i}-\mathrm{m}}$ & $66.21 \pm 0.15^{\mathrm{b}-\mathrm{f}}$ & $4.49 \pm 0.07^{\mathrm{op}, \mathrm{pq}}$ & $2580.75 \pm 123.10^{\mathrm{d}, \mathrm{e}}$ & 2 \\
\hline $\mathrm{CV}^{\# \#}$ & 9.97 & 20.32 & 14.96 & 3.25 & 8.34 & 5.60 & 13.25 & 1.74 & 3.31 & 7.10 & \\
\hline F value & ** & $* *$ & $* *$ & ** & ** & ** & ** & $* *$ & ** & ** & \\
\hline
\end{tabular}

Table 5. Variation in different nutritional attributes of selected indigenous aromatic rice genotypes. Different letters in the same line means statistical difference $(p<0.05)$ by Duncan test. CV means coefficient of variance. Statistical significance was measured by $\mathrm{F}$ test, ${ }^{\star *}$ means values are statistically significant at $\mathrm{p}<0.01$.

of conidia with disease incidence. The cultivars in present study have shown a range of adaptability to the blast disease. Our data suggest a significant negative correlation of leaf angle of the genotypes with occurrence of the disease. The leaves with smaller leaf angles may protect the spores from direct sunlight and favors the spore germinations $^{50,57}$ as direct sunlight has very detrimental effect to the germination of blast spore ${ }^{58}$. More over reduced leaf angles result in dense canopy cover and increases canopy temperature which may lead congenial micro-environment for the fungus and may positively influence the selective outbreak of the disease in these genotypes.

Search for resistant sources against blast disease has been going on all over the world and around 120 resistant genes have been reported till date. Majority of the pi genes are known to encode nucleotide binding site (NBS)-leucine rich repeats (LRR) proteins ${ }^{59}$. The genes which were found to be associated with blast tolerance of the genotypes in the present study, like $P i 9$ and pi 37 etc. are known to confer broad spectrum resistance to blast disease ${ }^{60,61}$. Pikh, pikm and pikp are known to be different allele of pik which act as a two-protein system in the plant against blast ${ }^{62}$. Pita and pizt are also known to exhibit complete resistance to blast disease. It can be 

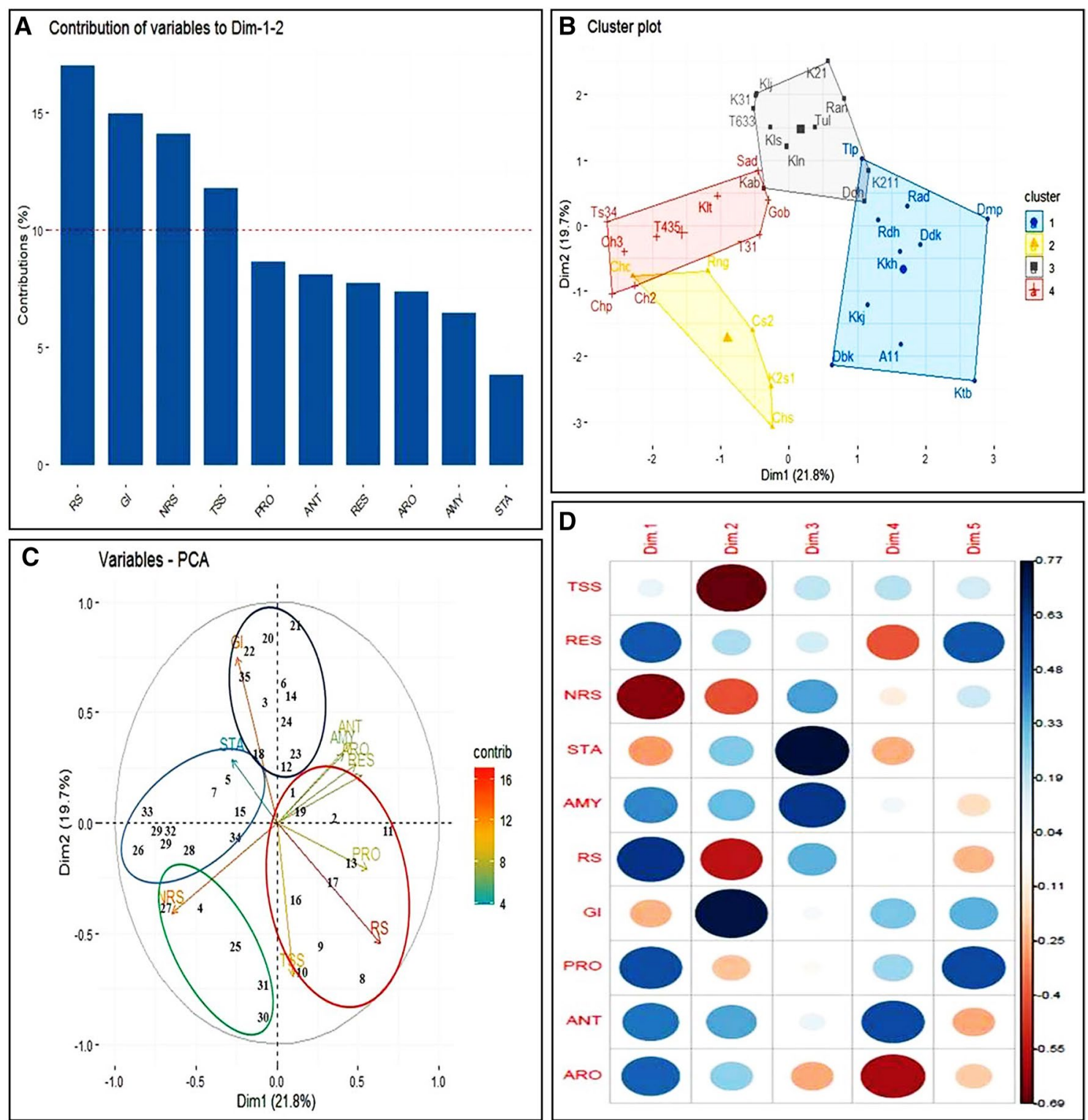

Figure 4. PCoA biplot of thirty-five genotypes based on their important nutritional attributes. All the analysis was performed using R software ${ }^{31}$, version 3.5.1, Patched (2018-07-02 r74950) Platform: x86_64w64mingw32/x64 (64-bit) (https://www.R-project.org/). (A) Contribution of various nutritional traits based on the mean values for the grouping of the rice genotypes. The Y-axis depicts the contribution of the parameters in percentage (\%). (B) Cluster analysis of the rice genotypes, the X and Y axis represent the PC1 (dim1) and PC2 (dim2) and the percentage (\%) of total variation defined by PC1 and PC2. (C) PCoA biplot exhibiting the grouping of the genotypes as well as the nutritional characters. The $\mathrm{X}$ and $\mathrm{Y}$ axis represent the PC1 (dim1) and PC2 (dim2) and the percentage (\%) of total variation defined by PC1 and PC2. (D) Dimension wise distribution of the nutritional characters contributing towards the clustering of the genotypes. The genotypes are denoted numerically or in short as follows 1. 'Tulaipanji' (Tlp), 2. 'Radhatilak' (Rad), 3. 'Kalshipa' (Kls), 4. 'Rangakomal' (Rng), 5. 'Sadanunia' (Sad), 6. 'Tulsibhog' (Tul), 7. 'Kalturey' (Klt), 8. 'Kataribhog' (Ktb), 9. 'A-1-1' (A11), 10. 'Dubarikomal' (Dbk), 11. 'Dharmaphou' (Dmp), 12. 'Dudheswar' (Ddh), 13. 'Dudhkalam' (Ddk), 14. 'Rampha' (Ran), 15. 'Gobindabhog' (Gob), 16. 'Konkanijhoha' (Kkj), 17. 'Kalokhasa' (Kkh), 18. 'Kabra' (Kab), 19. 'Radhunipagol' (Rdh), 20. 'Kalojeera' (Klj), 21. 'KNS-2'-1' (K2'1), 22. 'KNS-3'-1' (K3'1), 23. 'KNS-2-1-1' (K211), 24. 'Kalonunia' (Kln), 25. 'Chakhao sel-1' (Cs1), 26. 'Chakhao Poiterin' (Chp), 27. 'Chapka Chakhao' (Chc), 28. 'Chakhao sel-2' (Ch2), 29. 'Chakhao sel-3' (Ch3), 30. 'Chakhao Sampark' (Chs), 31. 'KNS-2B-S1' (K2s1), 32. 'T4M-3-5' (T435), 33. 'TSP6-M3-4' (TS34), 34. 'TPUR-B-1' (TSPM-3-1) (T31), 35. 'T6M-3-3' (T633). The attributes are described in short form as follows. TSS total soluble sugar, RES reducing sugar, NRS non-reducing sugar, STA starch, $A M Y$ amylose, $R S$ resistant starch, GI Glycemic Index, PRO protein, ANT antioxidant, $A R O$ aroma. 


\begin{tabular}{|c|c|c|c|c|c|c|c|c|c|c|c|c|}
\hline Genotypes & $\begin{array}{l}\text { Plant } \\
\text { height (cm) }\end{array}$ & Tiller number & $\begin{array}{l}\text { Lower leaf } \\
\text { angle }\left({ }^{\circ}\right)\end{array}$ & $\begin{array}{l}\text { Middle leaf } \\
\text { angle }\left(^{\circ}\right)\end{array}$ & $\begin{array}{l}\text { Upper leaf } \\
\text { angle }\left({ }^{\circ}\right)\end{array}$ & $\begin{array}{l}\text { Lesion no. in } \\
\text { lower leaf }\end{array}$ & $\begin{array}{l}\text { Lesion no. in } \\
\text { middle leaf }\end{array}$ & $\begin{array}{l}\text { Lesion no. in } \\
\text { upper leaf }\end{array}$ & Lesion type & $\begin{array}{l}\text { Lesion } \\
\text { size }\left(\mathrm{mm}^{2}\right)\end{array}$ & $\begin{array}{l}\text { Sporulation } \\
\text { centre }\end{array}$ & AUDPC for blast \\
\hline Tulaipanji & $110.37 \pm 0.52^{-\mathrm{g}}$ & $38.10 \pm 4.52^{\mathrm{a}}$ & $29.23 \pm 3.29^{\mathrm{b}-\mathrm{f}}$ & $15.33 \pm 5.13^{f}$ & $9.55 \pm 2.05^{e-f}$ & $4.07 \pm 2.12^{\mathrm{a}, \mathrm{b}}$ & $7.43 \pm 0.80^{a-c}$ & $11.68 \pm 2.19^{\mathrm{a}}$ & $1.31 \pm 0.01^{\mathrm{n}}$ & $1.26 \pm 0.006^{\circ, p}$ & $0.34 \pm 0.015^{i-k}$ & $922.01 \pm 130.77^{\mathrm{d}-\mathrm{k}}$ \\
\hline Radhatilak & $109.22 \pm 2.61^{\mathrm{d}-\mathrm{g}}$ & $33.62 \pm 2.94^{4, \mathrm{ab}}$ & $42.07 \pm 8.67^{\mathrm{a}}$ & $20.42 \pm 3.41^{b-f}$ & $9.50 \pm 0^{f}$ & $3.37 \pm 4.43^{\mathrm{a}, \mathrm{b}}$ & $4.47 \pm 4.90^{\mathrm{a}-\mathrm{e}}$ & $5.50 \pm 4.24^{\mathrm{b}-\mathrm{d}}$ & $1.28 \pm 0.01^{\mathrm{op}}$ & $1.41 \pm 0.010^{\mathrm{ij}}$ & $0.34 \pm 0.015^{i-k}$ & $873.60 \pm 30.16^{f-k}$ \\
\hline Kalshipa & $118.35 \pm 0.02^{a-g}$ & $33.72 \pm 1.00^{\mathrm{a}, \mathrm{b}}$ & $24.76 \pm 9.38^{\mathrm{eff}}$ & $15.35 \pm 3.46^{\mathrm{f}}$ & $10.70 \pm 1.08^{\mathrm{d}-\mathrm{f}}$ & $3.92 \pm 4.83^{\mathrm{a}, \mathrm{b}}$ & $4.30 \pm 4.66^{\mathrm{a}-\mathrm{e}}$ & $5.83 \pm 3.62^{b-d}$ & $1.70 \pm 0.01^{\mathrm{f}}$ & $2.25 \pm 0.028^{b}$ & $0.87 \pm 0.003^{b-d}$ & $819.01 \pm 86.87^{f-1}$ \\
\hline Rangakomal & $126.16 \pm 13.53^{a-c}$ & $24.48 \pm 0.91^{\mathrm{b}-\mathrm{h}}$ & $30.32 \pm 15.62^{b-f}$ & $19.30 \pm 4.57^{\mathrm{b}^{-\mathrm{f}}}$ & $13.55 \pm 0.44^{4-\mathrm{f}}$ & $2.87 \pm 3.72^{\mathrm{a}, \mathrm{b}}$ & $4.58 \pm 4.64^{a-e}$ & $6.10 \pm 3.91^{a-d}$ & $1.28 \pm 0.01^{\mathrm{op}, \mathrm{p}}$ & $1.54 \pm 0.010^{\mathrm{g}}$ & $0.51 \pm 0.011^{\mathrm{ghh}}$ & $758.32 \pm 59.97^{\mathrm{h}-1}$ \\
\hline Sadanunia & $111.87 \pm 2.99^{\mathrm{c}-\mathrm{g}}$ & $26.65 \pm 3.41^{\mathrm{b}-\mathrm{g}}$ & $32.72 \pm 0.02^{\mathrm{a}-\mathrm{f}}$ & $21.60 \pm 0.09^{\mathrm{g}-\mathrm{f}}$ & $16.32 \pm 2.28^{\mathrm{a}, \mathrm{b}}$ & $2.78 \pm 3.37^{\mathrm{a}, \mathrm{b}}$ & $4.32 \pm 4.54^{a-e}$ & $5.07 \pm 3.62^{b-d}$ & $1.00 \pm 0^{w}$ & $1.16 \pm 0.003^{s, t}$ & $0.62 \pm 0.014 \mathrm{f}^{-\mathrm{g}}$ & $421.69 \pm 83.96^{\mathrm{n}}$ \\
\hline Tulsibhog & $129.02 \pm 6.52^{\mathrm{a}, \mathrm{b}}$ & $26.22 \pm 6.34^{\mathrm{b}-\mathrm{g}}$ & $24.83 \pm 8.15^{\mathrm{e}, \mathrm{f}}$ & $18.03 \pm 3.81^{b-f}$ & $12.73 \pm 0.56^{-\mathrm{a}}$ & $2.65 \pm 3.27^{\mathrm{a}, \mathrm{b}}$ & $4.67 \pm 5.18^{\mathrm{a}-\mathrm{e}}$ & $6.02 \pm 4.36^{\mathrm{a}-\mathrm{d}}$ & $1.61 \pm 0.01^{\text {g.h }}$ & $1.43 \pm 0.009^{\mathrm{h}, \mathrm{i}}$ & $0.39 \pm 0.009^{h-j}$ & $644.19 \pm 16.41^{k-n}$ \\
\hline Kalturey & $113.10 \pm 7.73^{\mathrm{b}-\mathrm{g}}$ & $28.42 \pm 0.63^{3-\mathrm{f}}$ & $24.07 \pm 9.23^{f}$ & $20.67 \pm 1.79^{b-f}$ & $13.67 \pm 1.74^{4-\mathrm{f}}$ & $4.63 \pm 6.31^{\mathrm{a}}$ & $6.03 \pm 6.74^{\mathrm{a}, \mathrm{b}}$ & $7.50 \pm 6.59^{\mathrm{a}-\mathrm{d}}$ & $1.07 \pm 0.01^{v}$ & $1.46 \pm 0.010^{\mathrm{h}}$ & $0.26 \pm 0.006^{k, 1}$ & $880.35 \pm 45.99^{e-k}$ \\
\hline Kataribhog & $103.94 \pm 9.08^{\mathrm{gh}}$ & $31.07 \pm 1.79^{9-d}$ & $24.10 \pm 9.23^{\text {e.f }}$ & $16.45 \pm 1.3^{g e f f}$ & $11.38 \pm 4.69^{\mathrm{c}-\mathrm{f}}$ & $3.38 \pm 4.64^{\mathrm{a}, \mathrm{b}}$ & $4.23 \pm 5.23^{\mathrm{a}-\mathrm{e}}$ & $6.07 \pm 3.91^{\mathrm{a}-\mathrm{d}}$ & $1.30 \pm 0.01^{\mathrm{n}, \mathrm{o}}$ & $1.10 \pm 0.002^{u}$ & $0.07 \pm 0.001^{\mathrm{n}}$ & $909.42 \pm 10.24^{\mathrm{d}-\mathrm{k}}$ \\
\hline$A-1-1$ & $111.80 \pm 8.62^{\mathrm{c}-\mathrm{g}}$ & $28.15 \pm 6.10^{a-f}$ & $27.45 \pm 18.03^{c-f}$ & $20.80 \pm 9.66^{b-f}$ & $14.18 \pm 1.48^{a-e}$ & $2.42 \pm 3.18^{\mathrm{a}, \mathrm{b}}$ & $4.05 \pm 3.41^{a-e}$ & $5.03 \pm 3.48^{b-d}$ & $1.26 \pm 0.01^{\mathrm{p}, \mathrm{q}}$ & $1.52 \pm 0.010^{\mathrm{g}}$ & $0.43 \pm 0.009^{\mathrm{h}, \mathrm{i}}$ & $805.91 \pm 22.70^{\mathrm{d}-\mathrm{k}}$ \\
\hline Dubarikomal & $114.10 \pm 14.38^{\mathrm{a}-\mathrm{g}}$ & $23.53 \pm 0.51^{\mathrm{b}-\mathrm{h}}$ & $27.10 \pm 6.36^{c-f}$ & $17.90 \pm 0.70^{\mathrm{d}-\mathrm{f}}$ & $11.72 \pm 2.75^{b-f}$ & $4.02 \pm 4.83^{\mathrm{a}, \mathrm{b}}$ & $6.10 \pm 6.17^{\mathrm{a}, \mathrm{b}}$ & $8.77 \pm 6.45^{\mathrm{a}-\mathrm{c}}$ & $1.21 \pm 0.01^{\mathrm{s}, \mathrm{t}}$ & $1.13 \pm 0.003^{\text {tu, }}$ & $0.23 \pm 0.005^{\mathrm{k}, \mathrm{m}}$ & $1036.15 \pm 292.18^{g^{-1}}$ \\
\hline Darmaphou & $117.12 \pm 9.86^{6-8}$ & $24.18 \pm 3.74^{\mathrm{b}-\mathrm{h}}$ & $28.98 \pm 11.14^{1-f}$ & $20.95 \pm 4.83^{b-f}$ & $12.92 \pm 0.11^{\mathrm{a}-\mathrm{f}}$ & $2.02 \pm 2.56^{\mathrm{a}, \mathrm{b}}$ & $3.60 \pm 4.00^{b-e}$ & $6.35 \pm 5.06^{\mathrm{a}-\mathrm{d}}$ & $1.57 \pm 0.01^{i, j}$ & $1.56 \pm 0.030^{\mathrm{g}}$ & $0.82 \pm 0.019^{\text {die }}$ & $821.48 \pm 47.51^{a-h}$ \\
\hline Dudheswar & $122.71 \pm 7.66^{\text {a-e }}$ & $20.55 \pm 1.06^{\mathrm{e}-\mathrm{h}}$ & $24.80 \pm 10.13^{\mathrm{e}, \mathrm{f}}$ & $17.73 \pm 1.55^{\mathrm{b}-\mathrm{f}}$ & $12.03 \pm 1.13^{\mathrm{b}-\mathrm{f}}$ & $2.77 \pm 3.67^{\mathrm{a}, \mathrm{b}}$ & $4.15 \pm 4.73^{\text {be }}$ & $6.57 \pm 4.57^{\mathrm{a}-\mathrm{d}}$ & $1.80 \pm 0.01^{\mathrm{d}}$ & $1.92 \pm 0.021^{d}$ & $0.98 \pm 0.022^{\mathrm{a}, \mathrm{b}}$ & $1019.68 \pm 73.94^{4-1}$ \\
\hline Dudhkalam & $110.86 \pm 6.93^{\mathrm{c}-\mathrm{g}}$ & $21.47 \pm 2.07^{\mathrm{ch}}$ & $26.22 \pm 14.54^{\mathrm{d}-\mathrm{f}}$ & $19.10 \pm 4.85^{b-f}$ & $12.22 \pm 1.48^{a-f}$ & $3.45 \pm 4.64^{\mathrm{a}, \mathrm{b}}$ & $4.95 \pm 5.82^{\mathrm{a}-\mathrm{e}}$ & $7.60 \pm 5.37^{\mathrm{a}-\mathrm{d}}$ & $1.20 \pm 0.004^{t}$ & $1.26 \pm 0.006^{\mathrm{op}}$ & $0.13 \pm 0.003^{\mathrm{m}, \mathrm{n}}$ & $790.76 \pm 1.28^{\mathrm{b}-\mathrm{h}}$ \\
\hline Ranpha & $110.03 \pm 5.70^{\mathrm{c-g}}$ & $25.08 \pm 1.57^{\mathrm{b}-\mathrm{h}}$ & $24.63 \pm 9.28^{\mathrm{e}, \mathrm{f}}$ & $16.50 \pm 1.55^{\mathrm{e}^{\mathrm{ef}}}$ & $11.70 \pm 1.64^{b-f}$ & $3.83 \pm 4.66^{\mathrm{a}, \mathrm{b}}$ & $6.48 \pm 4.92^{\mathrm{a}-\mathrm{d}}$ & $9.02 \pm 4.54^{a-c}$ & $1.36 \pm 0.008^{\mathrm{m}}$ & $1.21 \pm 0.018^{q, x}$ & $0.20 \pm 0.004^{1, \mathrm{~mm}}$ & $1159.83 \pm 317.10^{g-1}$ \\
\hline Gobindabhog & $114.28 \pm 9.55^{\mathrm{a}-\mathrm{g}}$ & $29.30 \pm 2.21^{1-e}$ & $29.35 \pm 11.90^{b-f}$ & $20.88 \pm 3.13^{b-f}$ & $12.52 \pm 0.63^{3-f}$ & $5.40 \pm 7.35^{a}$ & $6.28 \pm 7.33^{\mathrm{a}-\mathrm{d}}$ & $8.67 \pm 7.91^{a-c}$ & $1.23 \pm 0.005^{\text {r.s }}$ & $1.31 \pm 0.015^{\mathrm{m}, \mathrm{n}}$ & $0.49 \pm 0.011^{\mathrm{ghh}}$ & $1309.86 \pm 38.54^{a-e}$ \\
\hline Konkanijoha & $115.44 \pm 7.48^{\mathrm{a}-\mathrm{g}}$ & $28.10 \pm 2.020^{\mathrm{a}-\mathrm{f}}$ & $24.12 \pm 5.8^{2, f}$ & $18.37 \pm 1.08^{c-f}$ & $11.28 \pm 1.90^{\mathrm{d}-\mathrm{f}}$ & $2.87 \pm 3.01^{\mathrm{a}, \mathrm{b}}$ & $4.00 \pm 3.53^{\mathrm{a}-\mathrm{e}}$ & $6.30 \pm 3.62^{\mathrm{a}-\mathrm{d}}$ & $1.57 \pm 0.009^{i . j}$ & $1.41 \pm 0.013^{\mathrm{i,j}}$ & $0.72 \pm 0.016^{\mathrm{eff}}$ & $1194.01 \pm 27.94^{\mathrm{a}}$ \\
\hline Kalokhasa & $120.87 \pm 11.39^{a-f}$ & $29.58 \pm 4.12^{2-e}$ & $28.52 \pm 12.42^{b-f}$ & $19.32 \pm 2.80^{b-f}$ & $12.63 \pm 2.12^{2-\mathrm{f}}$ & $2.40 \pm 3.25^{\mathrm{a}, \mathrm{b}}$ & $3.38 \pm 3.51^{c-e}$ & $4.48 \pm 3.27^{\mathrm{c}, \mathrm{d}}$ & $1.07 \pm 0.001^{v}$ & $1.28 \pm 0.016^{\mathrm{n}, \mathrm{o}}$ & $0.16 \pm 0.003^{1-n}$ & $971.10 \pm 71.61^{\mathrm{cit}}$ \\
\hline Kabra & $118.70 \pm 10.78^{\mathrm{a}-\mathrm{g}}$ & $31.82 \pm 1.33^{a^{-c}}$ & $29.67 \pm 5.65^{b-f}$ & $21.10 \pm 0.14^{b-f}$ & $11.68 \pm 1.67^{b-f}$ & $2.93 \pm 4.05^{\mathrm{a}, \mathrm{b}}$ & $4.32 \pm 5.35^{\mathrm{a}-\mathrm{e}}$ & $7.25 \pm 7.00^{a-d}$ & $1.25 \pm 0.01^{4, r}$ & $1.31 \pm 0.015^{\mathrm{m}, \mathrm{n}}$ & $0.16 \pm 0.003^{k-\mathrm{n}}$ & $792.49 \pm 6.52^{\mathrm{g}-1}$ \\
\hline Radhunipagol & $119.09 \pm 8.50^{0 \mathrm{a}-\mathrm{g}}$ & $25.40 \pm 1.50^{\mathrm{b}-\mathrm{h}}$ & $30.23 \pm 10.32^{b-f}$ & $22.92 \pm 4.40^{0-f}$ & $11.38 \pm 0.73^{c-f}$ & $2.42 \pm 3.04^{\mathrm{a}, \mathrm{b}}$ & $4.30 \pm 4.76^{\mathrm{a}-\mathrm{e}}$ & $7.10 \pm 5.98^{a-d}$ & $1.20 \pm 0.004^{t}$ & & $0.61 \pm 0.009^{f, g}$ & $960.64 \pm 205.6^{c-j}$ \\
\hline Kalojeera & $111.95 \pm 7.84^{\mathrm{c}-\mathrm{g}}$ & $26.37 \pm 7.63^{\mathrm{b}-\mathrm{g}}$ & $31.52 \pm 15.53^{\mathrm{a}-\mathrm{f}}$ & $20.35 \pm 1.72^{\mathrm{b}-\mathrm{f}}$ & $11.35 \pm 1.43^{\mathrm{d}-\mathrm{f}}$ & $3.52 \pm 4.50^{a, b}$ & $5.32 \pm 5.06^{\mathrm{a}-\mathrm{e}}$ & $8.12 \pm 5.77^{\mathrm{a}-\mathrm{c}}$ & $1.10 \pm 0.002^{\mathrm{u}}$ & $1.33 \pm 0.038^{\mathrm{lm}}$ & $0.28 \pm 0.016^{i-1}$ & $1096.76 \pm 88.62^{a-f}$ \\
\hline Chakhao sel-1 & $113.99 \pm 19.94^{a-g}$ & $31.48 \pm 7.00^{a-d}$ & $34.12 \pm 16.14^{a-f}$ & $22.22 \pm 5.86^{6^{-f}}$ & $12.90 \pm 1.17^{\mathrm{z}-\mathrm{f}}$ & $5.50 \pm 7.40^{\mathrm{a}}$ & $6.30 \pm 7.21^{\mathrm{a}-\mathrm{d}}$ & $9.25 \pm 9.12^{a-c}$ & $1.74 \pm 0.006^{e}$ & $1.82 \pm 0.019^{e}$ & $0.97 \pm 0.0007^{\mathrm{a}-\mathrm{c}}$ & $980.15 \pm 84.42^{c-i}$ \\
\hline $\begin{array}{l}\text { Chakhao } \\
\text { Poiterein }\end{array}$ & $126.48 \pm 21.99^{a-c}$ & $16.37 \pm 2.26^{\mathrm{g}, \mathrm{h}}$ & $35.20 \pm 14.56^{\mathrm{a}-\mathrm{f}}$ & $25.20 \pm 7.44^{\mathrm{a}, \mathrm{b}}$ & $13.65 \pm 2.09^{-\mathrm{a}}$ & $4.17 \pm 5.70^{\mathrm{a}, \mathrm{b}}$ & $4.95 \pm 6.38^{\text {a-e }}$ & $6.75 \pm 6.24^{a-d}$ & $1.46 \pm 0.01^{\mathrm{k}}$ & $1.25 \pm 0.010^{0-q}$ & $0.51 \pm 0.011^{\mathrm{ghh}}$ & $728.92 \pm 190.5^{\mathrm{i}-\mathrm{m}}$ \\
\hline $\begin{array}{l}\text { Chapka } \\
\text { Chakhao }\end{array}$ & $116.57 \pm 7.21^{1-\mathrm{g}}$ & $15.37 \pm 1.79^{\mathrm{h}}$ & $36.37 \pm 0.23^{\mathrm{a}-\mathrm{d}}$ & $23.40 \pm 4.43^{-\mathrm{ae}}$ & $13.63 \pm 0.56^{-\mathrm{af}}$ & $3.10 \pm 4.33^{\mathrm{a}, \mathrm{b}}$ & $4.50 \pm 4.76^{\mathrm{a}-\mathrm{e}}$ & $6.92 \pm 7.18^{a-d}$ & $1.97 \pm 0.0007^{\mathrm{a}}$ & $2.38 \pm 0.031^{\mathrm{a}}$ & $1.00 \pm 0^{\mathrm{a}}$ & $864.71 \pm 6.87^{-\mathrm{f-k}}$ \\
\hline Chakhao sel-2 & $129.98 \pm 16.52^{\mathrm{a}}$ & $22.45 \pm 7.18^{\mathrm{ch}}$ & $36.88 \pm 10.44^{a-d}$ & $29.30 \pm 6.22^{\mathrm{a}}$ & $16.02 \pm 3.79^{a-c}$ & $2.77 \pm 3.81^{\mathrm{a}, \mathrm{b}}$ & $3.90 \pm 4.43^{\mathrm{a}-\mathrm{e}}$ & $5.52 \pm 5.35^{\mathrm{b}-\mathrm{d}}$ & $1.10 \pm 0.002^{\mathrm{u}}$ & $1.31 \pm 0.015^{\mathrm{m}, \mathrm{n}}$ & $0.67 \pm 0.007^{\mathrm{f}}$ & $776.27 \pm 257.47^{\mathrm{h}-1}$ \\
\hline Chakhao sel-3 & $124.87 \pm 17.52^{\mathrm{a}-\mathrm{d}}$ & $20.25 \pm 2.61^{\mathrm{e}-\mathrm{h}}$ & $39.63 \pm 11.17^{\mathrm{a}, \mathrm{b}}$ & $26.72 \pm 3.60^{\mathrm{a}, \mathrm{b}}$ & $12.62 \pm 0.73^{\mathrm{a}-\mathrm{f}}$ & $3.93 \pm 5.56^{\mathrm{a}, \mathrm{b}}$ & $4.85 \pm 5.86^{a-e}$ & $6.25 \pm 6.52^{\mathrm{a}-\mathrm{d}}$ & $1.26 \pm 0.006^{p, q}$ & $1.36 \pm 0.038^{\mathrm{k}, 1}$ & $0.21 \pm 0.018^{\mathrm{k}-\mathrm{m}}$ & $700.35 \pm 139.86^{i-m}$ \\
\hline Kalonunia & $104.49 \pm 1.32^{i-h}$ & $25.18 \pm 8.17^{\mathrm{b}-\mathrm{h}}$ & $26.08 \pm 5.63^{\mathrm{d}-\mathrm{f}}$ & $19.37 \pm 1.22^{b-f}$ & $11.85 \pm 3.27^{\mathrm{b}-\mathrm{f}}$ & $4.27 \pm 5.75^{\mathrm{a}, \mathrm{b}}$ & $6.00 \pm 7.30^{a-d}$ & $8.33 \pm 7.87^{\mathrm{a}-\mathrm{c}}$ & $1.56 \pm 0.01^{j}$ & ${ }_{\mathrm{m}}^{1.34 \pm 0.015^{\mathrm{k}-}}$ & $0.80 \pm 0.004^{\mathrm{d}, e}$ & $1169.96 \pm 26.08^{a-d}$ \\
\hline KNS-2'-1 & $108.69 \pm 3.64^{\mathrm{d}-\mathrm{g}}$ & $16.77 \pm 2.30^{\mathrm{ghh}}$ & $32.28 \pm 13.22^{\mathrm{a}-\mathrm{f}}$ & $23.20 \pm 4.19^{\mathrm{a}-\mathrm{f}}$ & $12.15 \pm 1.57^{\mathrm{a}-\mathrm{f}}$ & $4.07 \pm 5.27^{\mathrm{a}, \mathrm{b}}$ & $6.48 \pm 6.81^{\mathrm{a}-\mathrm{d}}$ & $8.65 \pm 7.14^{\mathrm{a}-\mathrm{c}}$ & $1.39 \pm 0.009^{1}$ & $1.23 \pm 0.005^{\mathrm{p}-\mathrm{r}}$ & $0.61 \pm 0.009^{f, g}$ & $1076.83 \pm 57.41^{a-g}$ \\
\hline $\begin{array}{l}\text { KNS-3'-1 Uttar } \\
\text { Sugandhi (IET } \\
24,616)\end{array}$ & $107.82 \pm 2.29^{\mathrm{e}-\mathrm{g}}$ & $22.05 \pm 3.74^{c-h}$ & $30.17 \pm 10.98^{b-f}$ & $19.60 \pm 0.61^{\mathrm{b}-\mathrm{f}}$ & $12.77 \pm 1.93^{3-\mathrm{f}}$ & $5.18 \pm 6.67^{\mathrm{a}}$ & $7.58 \pm 6.85^{\mathrm{ab}}$ & $9.40 \pm 7.87^{\mathrm{a}-\mathrm{c}}$ & $1.36 \pm 0.008^{\mathrm{m}}$ & $1.28 \pm 0.016^{\mathrm{n}, \mathrm{o}}$ & $0.67 \pm 0.007 \mathrm{f}$ & $1265.32 \pm 31.90^{0 \mathrm{a}, \mathrm{b}}$ \\
\hline KNS-2-1-1 & $116.51 \pm 5.12^{\mathrm{a}-\mathrm{g}}$ & $18.10 \pm 1.08^{\mathrm{f}-\mathrm{h}}$ & $32.70 \pm 11.64^{a-f}$ & $21.82 \pm 0.44^{\mathrm{a}-\mathrm{f}}$ & $11.17 \pm 1.08^{\mathrm{b}-\mathrm{f}}$ & $5.70 \pm 7.91^{a}$ & $8.02 \pm 9.07^{\mathrm{a}}$ & $10.65 \pm 9.73^{\mathrm{a}, \mathrm{b}}$ & $1.59 \pm 0.01^{\mathrm{h}, \mathrm{i}}$ & $1.21 \pm 0.018^{q, r}$ & $0.66 \pm 0.015 f$ & $914.44 \pm 141.49^{\mathrm{d}-\mathrm{k}}$ \\
\hline KNS-2B-S1 & $111.92 \pm 3.50^{\mathrm{c}-\mathrm{g}}$ & $20.40 \pm 1.08^{-\mathrm{eh}}$ & $35.27 \pm 10.41^{\mathrm{a}-\mathrm{e}}$ & $23.00 \pm 0.28^{-\mathrm{a} f}$ & $11.68 \pm 1.34^{\mathrm{d}-\mathrm{f}}$ & $4.12 \pm 5.49^{\mathrm{a}, \mathrm{b}}$ & $5.70 \pm 6.45^{\mathrm{a}-\mathrm{d}}$ & $9.55 \pm 7.14^{a-c}$ & $1.62 \pm 0.01^{\mathrm{g}}$ & $1.38 \pm 0.014^{j, k}$ & $0.66 \pm 0.015^{f}$ & $1229.66 \pm 115.87^{7-6}$ \\
\hline $\begin{array}{l}\text { Chakhao } \\
\text { Sampark }\end{array}$ & $118.57 \pm 0.34^{a-g}$ & $21.43 \pm 13.52^{c-h}$ & $33.10 \pm 3.58^{\mathrm{a}-\mathrm{f}}$ & $24.72 \pm 1.76^{\mathrm{a}, \mathrm{b}}$ & $14.57 \pm 1.41^{\mathrm{a}, \mathrm{b}}$ & $0.50 \pm 0.70^{\mathrm{b}}$ & $1.33 \pm 1.17^{\mathrm{e}}$ & $1.93 \pm 0.09^{\mathrm{b}}$ & $1.10 \pm 0.002^{\mathrm{u}}$ & $1.20 \pm 0.004^{4 s}$ & $0.43 \pm 0.329^{\mathrm{h}, \mathrm{i}}$ & $469.20 \pm 43.55^{\mathrm{m}, \mathrm{n}}$ \\
\hline $\begin{array}{l}\text { TSPM-3-1 } \\
\text { TPUR-B-1(IET } \\
28,104)\end{array}$ & $92.12 \pm 5.21^{\mathrm{h}-\mathrm{i}}$ & $20.62 \pm 0.73^{\mathrm{e}-\mathrm{h}}$ & $37.85 \pm 6.85^{a-c}$ & $19.62 \pm 5.11^{b-f}$ & $13.75 \pm 1.62^{2-f}$ & $1.87 \pm 2.63^{\mathrm{ab}, \mathrm{b}}$ & $5.43 \pm 6.83^{\mathrm{a}-\mathrm{e}}$ & $4.82 \pm 4.40^{\mathrm{c}, \mathrm{d}}$ & $1.89 \pm 0.02^{\mathrm{b}}$ & $2.18 \pm 0.027^{c}$ & $0.85 \pm 0.019^{\mathrm{c}, \mathrm{d}}$ & $676.96 \pm 52.05^{j-\mathrm{n}}$ \\
\hline T4M-3-5 & $85.80 \pm 0.28^{\mathrm{i}}$ & $17.15 \pm 0.54^{\mathrm{ghh}}$ & $35.17 \pm 0.80^{a-f}$ & $29.05 \pm 3.88^{\mathrm{a}}$ & $16.72 \pm 3.46^{\mathrm{a}}$ & $3.18 \pm 4.31^{\mathrm{a}, \mathrm{b}}$ & $2.75 \pm 3.08^{\mathrm{d}, e}$ & $5.23 \pm 5.79^{b-d}$ & $1.85 \pm 0.01^{\mathrm{c}}$ & $1.36 \pm 0.008^{\mathrm{k}, 1}$ & $0.61 \pm 0.009 f \cdot \mathrm{g}$ & $558.63 \pm 40.06^{1-n}$ \\
\hline T6M-3-3 & $80.40 \pm 1.45^{\mathrm{i}}$ & $21.22 \pm 5.53^{\mathrm{d}-\mathrm{h}}$ & $34.48 \pm 5.25^{\mathrm{a}-\mathrm{f}}$ & $26.08 \pm 0.91^{-a c}$ & $14.90 \pm 1.13^{3-d}$ & $3.60 \pm 4.99^{\mathrm{a}, \mathrm{b}}$ & $4.28 \pm 4.97^{\mathrm{a}-\mathrm{e}}$ & $7.08 \pm 7.33^{\mathrm{a}-\mathrm{d}}$ & $1.98 \pm 0.02^{\mathrm{a}}$ & $1.67 \pm 0.007 \mathrm{f}$ & $0.61 \pm 0.009^{f-8}$ & $783.51 \pm 129.38^{\mathrm{h}-1}$ \\
\hline TSP6-M3-4 & $81.83 \pm 1.17^{\mathrm{i}}$ & $21.73 \pm 0.04^{c^{-h}}$ & $34.60 \pm 10.13^{\mathrm{a}-\mathrm{f}}$ & $26.65 \pm 3.46^{a, b}$ & $13.77 \pm 1.64^{4-\mathrm{f}}$ & $3.10 \pm 4.19^{\mathrm{a}, \mathrm{b}}$ & $4.57 \pm 5.70^{\mathrm{a}-\mathrm{e}}$ & $6.50 \pm 6.92^{\mathrm{a}-\mathrm{d}}$ & $1.98 \pm 0.02^{\mathrm{a}}$ & $1.80 \pm 0.004^{e}$ & $0.90 \pm 0.002^{2-d}$ & $642.29 \pm 19.09^{k-n}$ \\
\hline $\mathrm{CV}^{s p}$ & 4.25 & 12.07 & 10.40 & 10.64 & 10.47 & 31.86 & 23.97 & 23.61 & 0.59 & 0.89 & 7.52 & 9.46 \\
\hline F value & $* *$ & ** & - & * & - & - & - & - & $* *$ & $* *$ & $* *$ & ** \\
\hline
\end{tabular}

Table 6. Variation in plant morphological and disease related characters of selected indigenous aromatic rice genotypes. CV means Coefficient of Variance, Statistical significance was measured by F test, ${ }^{* *}$ means values are statistically significant at $\mathrm{p}<0.01$.

presumed that the landraces have acquired this resistant gene while combating continuous pathogen pressure for a long period of time. Along with these known genes the presence of unknown genes may also be contributing to the resistance of the genotypes against the blast disease. Detail investigation of the resistant genotypes may lead to the isolation of novel genes or QTLs linked with blast disease resistance.

\section{Conclusion}

In the current study, proximate analysis of non-Basmati aromatic rice genotypes has been performed which has successfully zeroed on cultivars like, 'Kataribhog, 'Sadanunia,' 'Kalshipa' etc. having low glycemic index, high resistant starch and high antioxidant potential respectively. On the basis of collective evidence from two years field trial and in vitro experiments blast resistant local genotypes like 'Sadanunia, 'Chakhao Sampark, 'T4M-3-5' etc. were identified. Allele mining for the resistant genes in these genotypes demonstrated significant association of six pi genes with resistance against blast disease. The prediction model with plant morphological characters 
A
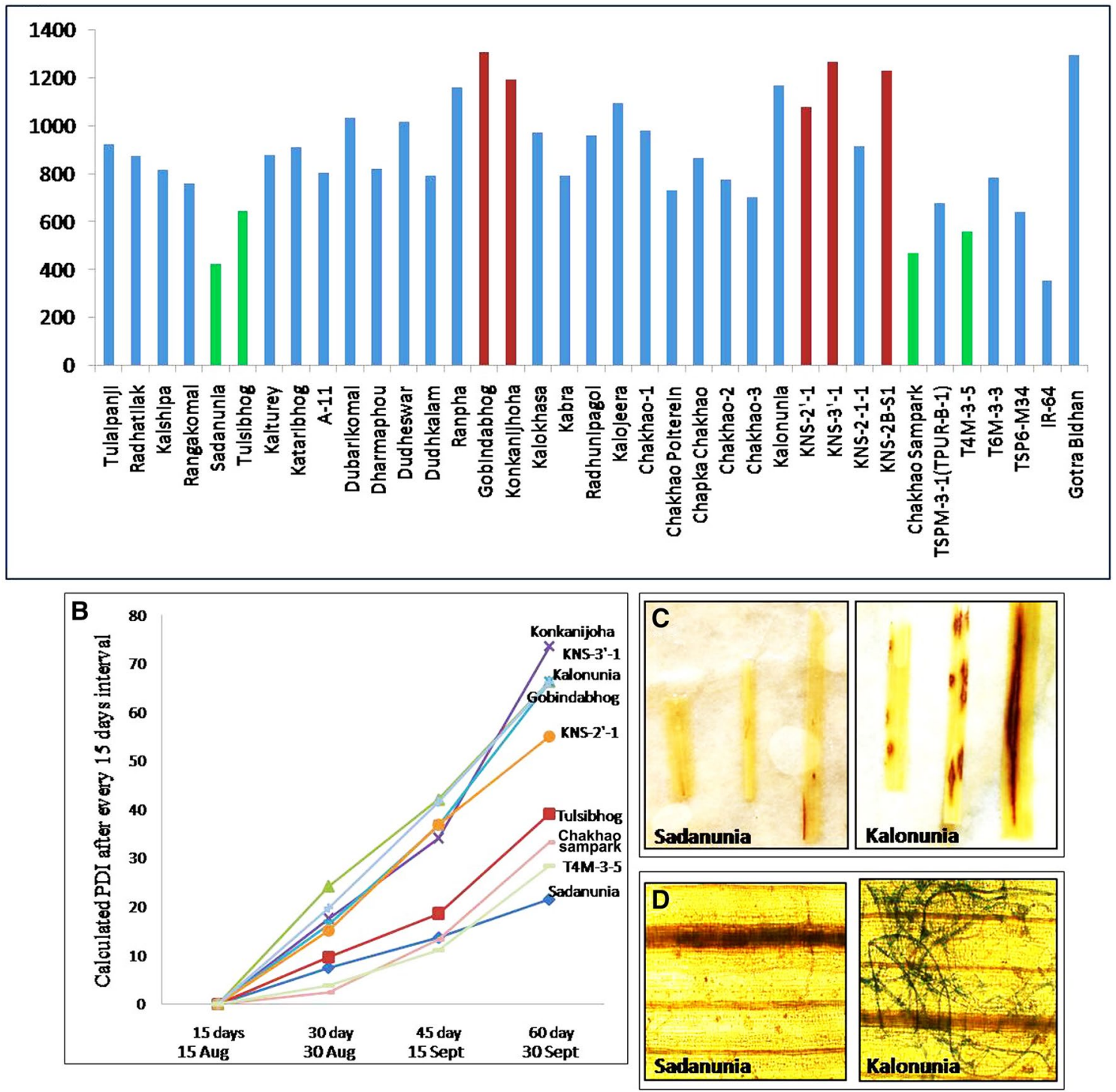

Figure 5. Screening of the local landraces for the occurrence of the leaf last disease. (A) Calculated AUDPC of all the genotypes from 2 year's field trial and scoring of the plants for disease symptoms. (B) PDI of some highly susceptible and tolerant cultivars between mid of August to early October. (C) Time lapse photography of the leaves from susceptible ('Kalonunia') and tolerant ('Sadanunia') cultivars after inoculation from purified blast fungus. (D) Microscopic image of hyphal growth in susceptible ('Kalonunia') and tolerant ('Sadanunia') cultivars. 

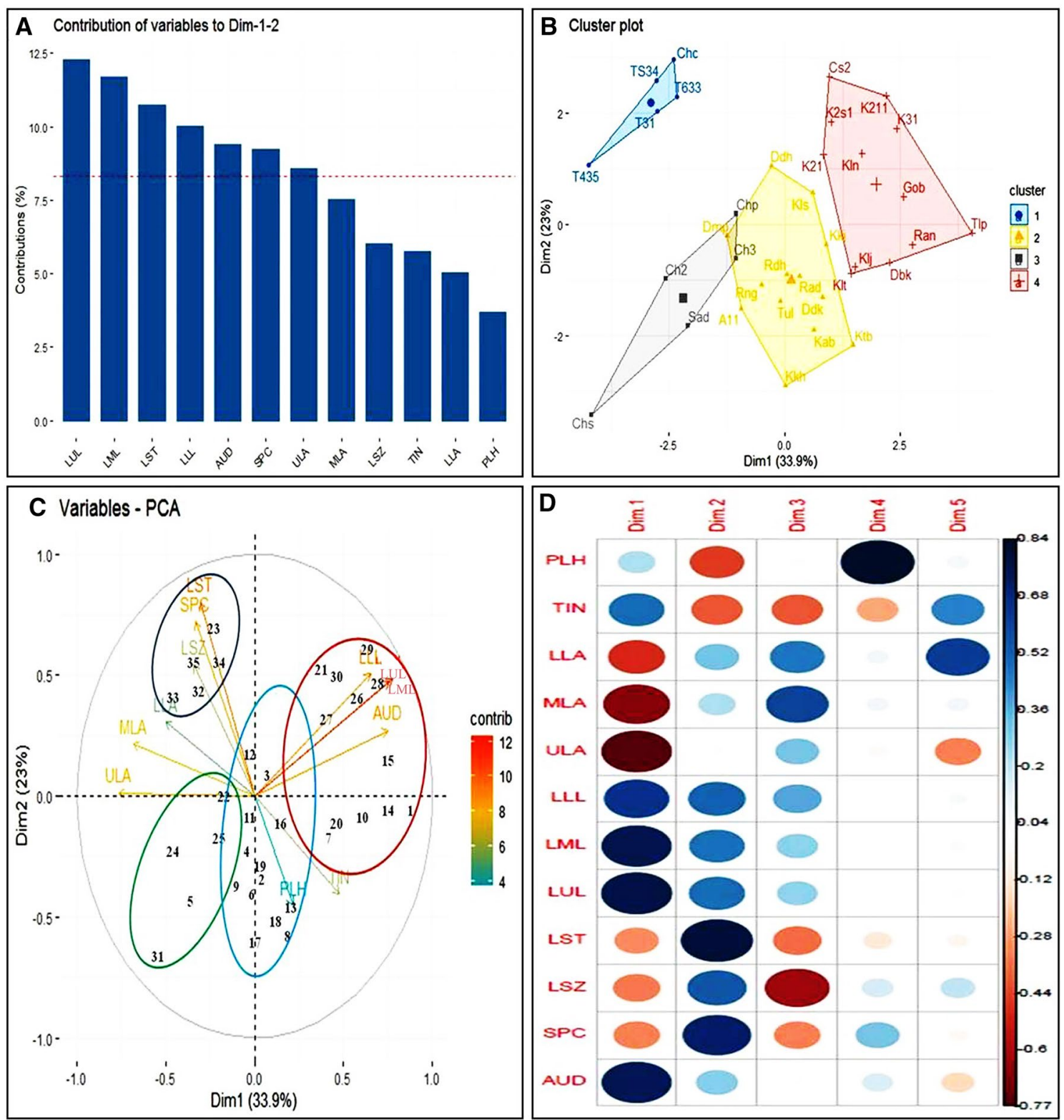

Figure 6. PCoA biplot of thirty-five genotypes based on their important phenotypic and disease related attributes. All the analysis was performed using R software ${ }^{31}$, version 3.5.1, Patched (2018-07-02 r74950) Platform: x86_64-w64mingw32/x64 (64-bit) (https://www.R-project.org/). (A) Contribution of various phenotypic and disease related attributes based on the mean values for the grouping of the rice genotypes. The Y-axis depicts the contribution of the parameters in percentage (\%). (B) Cluster analysis of the rice genotypes, the $\mathrm{X}$ and $\mathrm{Y}$ axis represent the PC1 (dim1) and PC2 (dim2) and the percentage (\%) of total variation defined by PC1 and PC2. (C) PCoA biplot exhibiting the grouping of the genotypes as well as the phenotypic and disease related attributes. The X and Y axis represent the PC1 (dim1) and PC2 (dim2) and the percentage (\%) of total variation defined by PC1 and PC2. (D) Dimension wise distribution of the phenotypic and disease related attributes contributing towards the clustering of the genotypes. The genotypes are designated either numerically or in short form as following 1. 'Tulaipanji' (Tlp), 2. 'Radhatilak' (Rad), 3. 'Kalshipa' (Kls), 4. 'Rangakomal' (Rng), 5. 'Sadanunia' (Sad), 6. 'Tulsibhog' (Tul), 7. 'Kalturey' (Klt), 8. 'Kataribhog' (Ktb), 9. 'A-1-1' (A11), 10. 'Dubarikomal' (Dbk), 11. 'Dharmaphou' (Dmp), 12. 'Dudheswar' (Ddh), 13. 'Dudhkalam' (Ddk), 14. 'Rampha' (Ran), 15. 'Gobindabhog' (Gob), 16. Konkanijhoha (Kkj), 17. Kalokhasa (Kkh), 18. Kabra (Kab), 19. Radhunipagol (Rdh), 20. Kalojeera (Klj), 21. Chakhao Sel.-1 (Cs1), 22. Chakhao Poiterin (Chp), 23. Chapka Chakhao (Chc), 24. Chakhao sel-2 (Ch2), 25. Chakhao sel-3 (Ch3), 26. Kalonunia (Kln), 27. KNS-2'-1 (K2'1), 28. KNS-3'-1 (K3'1), 29. KNS-2-1-1 (K211), 30. KNS-2B-S1 (K2s1), 31. Chakhao Sampark (Chs), 32. TPURB-1(TSPM-3-1) (T31), 33. T4M-3-5 (T435), 34. T6M-3-3 (T633), 35. TSP6-M3-4 (TS34). The phenotypic attributes are denoted as $P L H$ plant height, TIN Tiller Number, $L L A$ lower leaf angle, $M L A$ middle leaf angle, $U L A$ upper leaf angle, $L L L$ lesion no. In Lower Leaf, $L M L$ lesion no. In Middle Leaf, $L U L$ lesion no. In Upper Leaf, $L S T$ lesion type, $L S Z$ lesion size, SPC sporulation centre, AUD AUDPC. 


\begin{tabular}{|c|c|c|c|c|c|c|c|c|c|c|c|c|}
\hline \multicolumn{13}{|l|}{ Correlations } \\
\hline & Plh & Tin & Lla & Mla & Ula & LII & Lml & Lul & Lst & Lss & Spc & AUDPC \\
\hline \multicolumn{13}{|l|}{ AUDPC } \\
\hline Pearson correlation & 0.078 & 0.145 & -0.183 & $-0.261^{\star}$ & $-0.348^{* *}$ & 0.124 & 0.149 & $0.236^{*}$ & -0.040 & -0.123 & 0.014 & 1 \\
\hline Sig. (2-tailed) & 0.521 & 0.232 & 0.130 & 0.029 & \begin{tabular}{|l|}
0.003 \\
\end{tabular} & 0.306 & 0.220 & \begin{tabular}{|l|l|}
0.049 \\
\end{tabular} & 0.743 & 0.309 & 0.909 & \\
\hline
\end{tabular}

Table 7. Correlation and regression analysis of the morphological and disease related characters with AUDPC. ${ }^{\star}$ Correlation is significant at the 0.05 level (2-tailed). ${ }^{*}$ Correlation is significant at the 0.01 level (2-tailed). PLH plant height, TLN Tiller number, $L L A$ lower leaf angle, MLA middle leaf angle, ULA upper leaf angle, $L L L$ lesion number in lower leaf, $L M L$ lesion number in middle leaf, $L U L$ lesion number in upper leaf, LST lesion type, LSS lesion size, Spc sporulation center.

\begin{tabular}{|c|c|c|c|c|c|c|c|}
\hline Sl. no. & Gene name & Forward sequence & Reverse sequence & Chromosome no. & Amplicon size (bp) & $\begin{array}{l}\text { Annealing } \\
\text { temp }\left({ }^{\circ} \mathrm{C}\right)\end{array}$ & References \\
\hline 1. & Pi-d2 & TTGGCTATCATAGGCGTCC & ATTTGAAGGCGTTTGCGTAGA & 6 & 1057 & 55 & 63 \\
\hline 2. & Pi-36 & CAATGTGTGACTTGTGCGGACT & TCTTCCATCTCGGATTTCGTGT & 8 & 1036 & 55 & 64 \\
\hline 3. & Pi-37 & TCTTGAGGGTCCCAGTGTAC & CGAACAGTGGCTGGTATCTC & 1 & 1149 & 55 & 65 \\
\hline 4. & Pi5 & TCCTCCTCTTCGGACACCTC & CGGACGAGCGATAGTGATCC & 9 & 594 & 55 & 65 \\
\hline 5. & Pi-z & $\begin{array}{l}\text { GGACCCGCGTTTTCCACG } \\
\text { TGTAA }\end{array}$ & $\begin{array}{l}\text { AGGAATCTATTGCTAAGC } \\
\text { ATGAC }\end{array}$ & 6 & 292 & 60 & 66 \\
\hline 6. & Piz-t & TTGCTGAGCCATTGTTAAACA & $\begin{array}{l}\text { ATCTCTTCATATATATGAAGG } \\
\text { CCAC }\end{array}$ & 6 & 257 & 56 & 67 \\
\hline 7. & Pik-p & $\begin{array}{l}\text { ATAGTTGAATGTATGGAATGG } \\
\text { AAT }\end{array}$ & CTGCGCCAAGCAATAAAGTC & 11 & 148 & 60 & 67 \\
\hline 8. & Pik-h & $\begin{array}{l}\text { CATGAGTTCCATTTACTATTC } \\
\text { CTC }\end{array}$ & $\begin{array}{l}\text { ACATTGGTAGTAGTGCAA } \\
\text { TGTCA }\end{array}$ & 11 & 1500 & 55 & 68 \\
\hline 9. & Pi-b & GACTCGGTCGACCAATTCGCC & ATCAGGCCAGGCCAGATTTG & 2 & 388 & 60 & 67 \\
\hline 10. & Pi-9 & ATGGTCCTTTATCTTTATTG & TTGCTCCATCTCCTCTGTT & 6 & 2000 & 53 & 61 \\
\hline 11. & Pi-ta/Pi-ta2 & AGCAGGTTATAAGCTAGGCC & CTACCAACAAGTTCATCAAA & 12 & 1042 & 58 & 69 \\
\hline 12. & Pik & $\begin{array}{l}\text { GCCACATCAATGGCTACA } \\
\text { ACGTT }\end{array}$ & CCAGAATTTACAGGCTCTGG & 11 & 112 & 60 & 67 \\
\hline 13. & Pi2-1 & GATTTAGTTCAGGAAAACACTC & TGGAAGCCTCATTGATCATC & 12 & 2344 & 55 & 70 \\
\hline 14. & Pi2-2 & CGTTGTATAGGACAGTTTCATT & AATCTAGGCACTCAAGTGTTC & 6 & 436 & 50 & 71 \\
\hline 15. & Pi2-3 & CAGCGATGGTATGAGCACAA & CGTTCCTATACTGCCACATCG & 5 & 450 & 57 & 72 \\
\hline 16. & Pi-1 & GTGTAAATCATGGGCACGTG & AGATTGGCTCCTGAAGAAGG & 11 & 170 & 55 & 73 \\
\hline 17. & Pik-m & CGTGCTGTCGCCTGAATCTG & CACGAACAAGAGTGTGTCGG & 11 & 619 & 55 & 74 \\
\hline 18. & $\operatorname{Pi}-61(\mathrm{t})$ & AGATGATAAGCTTGCGGACC & ATGCAGATGAGTCCCTCCAC & 11 & 210 & 55 & 75 \\
\hline 19. & $\mathrm{Pi}-2$ & CTCCTTCAGCTGCTCCTC & TGATGACTTCCAAACGGTAG & 6 & 200 & 58 & 76 \\
\hline 20. & Pik & CGTGCTGTCGCCTGAATCTG & CACGAACAAGAGTGTGTCGG & 11 & 150 & 58 & 76 \\
\hline 21. & Pi7t & CACTCACACGAACGACTGAC & CGCAGGTTCTTGTGAAATGT & 11 & 200 & 56 & 76 \\
\hline 22. & Pi-33 & \multicolumn{2}{|l|}{ Motif $=($ TAT $) 5 C($ ATT $) 15$} & 8 & 166 & 56 & 77 \\
\hline 23. & $\operatorname{Pi}-27(t)$ & \multicolumn{2}{|l|}{ Motif $=(\mathrm{CT}) 17$} & 1 & 162 & 56 & 76 \\
\hline
\end{tabular}

Table 8. Details of $P i$ genes analysed in the study.

were developed with an accuracy level of more than $85 \%$ for the occurrence of blast disease. The scope for pushing non-Basmati scented rice in the domestic as well as the global market is growing and it is high time to highlight and popularize these folk cultivars for their nutritional and disease resistance attributes. Many of these traditional cultivars may also be used as donors for traits like biotic, abiotic stress resistance and for aroma in rice improvement programs. In addition, the use of these cultivars as donor will result in large number of segregants in subsequent generation due to the wide genetic base of these cultivars. It is also important to build strategies for improvement of these genotypes in terms of yield, photosensitivity, disease resistance, cooking quality, and benefit to human health etc. using mutation breeding or biotechnological tools keeping the desirable traits like aroma intact. Combined approaches for the betterment of these heirloom rice cultivars will encourage the farmers to take on the cultivation of their own traditional genotypes over the HYVs. 


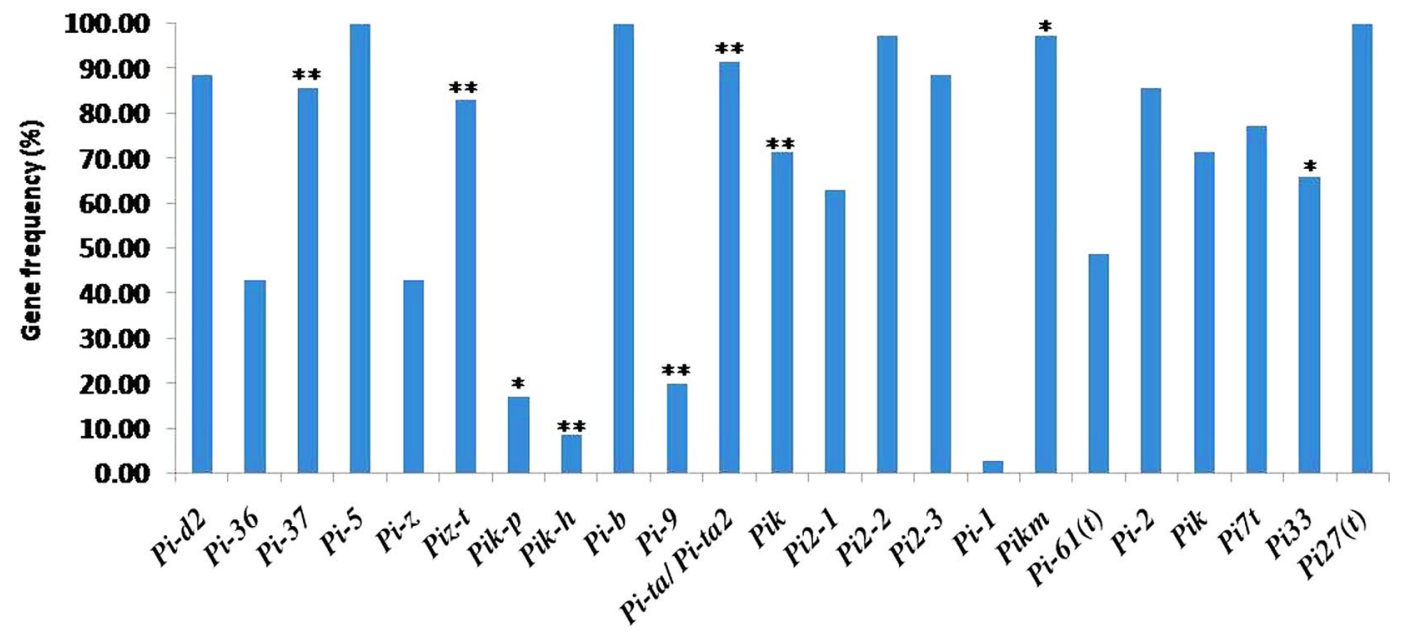

Figure 7. Distribution of twenty-three major blast resistant genes in the local landraces. Frequency of the genes in the landraces is calculated. Significant Kendall's-tau b association of the Pi gene with tolerance to the disease is represented by '*' (** mean level of significance $<0.01$, ${ }^{*}$ means level of significance $\left.<0.05\right)$. Kendall's tau-b correlation coefficient was calculated using IBM-SPSS trial version $20^{33}$ (https://www.ibm.com/analytics/spssstatistics-software).

\begin{tabular}{|l|l|l|l|l|}
\hline Parameters & B & S.E. & Sig & $\operatorname{Exp(B)}$ \\
\hline Lesion number in upper leaf (LUL) & 0.892 & 0.332 & 0.007 & 2.439 \\
\hline Lesion number in middle leaf (LML) & -0.759 & 0.463 & 0.049 & 0.468 \\
\hline Lesion type (LST) & -2.845 & 1.467 & 0.061 & 0.058 \\
\hline Lesion number in lower leaf (LLL) & -0.144 & 0.421 & 0.070 & 0.866 \\
\hline Sporulation center (SPC) & 2.847 & 1.526 & 0.057 & 17.244 \\
\hline Upper leaf angle (ULA) & -0.329 & 0.149 & 0.027 & 0.720 \\
\hline Constant & 5.566 & 2.776 & 0.045 & 261.457 \\
\hline
\end{tabular}

Table 9. The logistic regression model was fitted by considering only significant variables except AUDPC based on PCA results. Where B signifies coefficient for fitted logistic regression model, SE means standard error of the parameter estimate, Sig. indicates $\mathrm{P}$ values and EXP(B) signifies odd in favorable Blast Occurrence. Here, based on AUDPC values, disease occurrence has been calculated as 1 when AUDPC $>800$.

Received: 30 October 2020; Accepted: 3 February 2021

Published online: 26 February 2021

\section{References}

1. Chatterjee, P. Whose rice is it anyway? https://www.livemint.com/mintlounge/features/whose-rice-is-it-anyway-1564127848906. html (2019).

2. Mahato, S., Surje, D. T., Debbarma, S. \& Roy, B. Characterization of some aromatic farmers' varieties of rice (Oryzasativa L.) from West Bengal and adjoining states. Indian J. Plant Genet. Resour. 30(2), 120-129. https://doi.org/10.5958/0976-1926.2017.00018.3 (2017).

3. Sanyal, C.C. The Rajbanshis of North Bengal. The Asiatic Society Monograph Ser. 9 (1965).

4. Dasgupta, A. Ontology and Epistemology of Tribal Groups in North Bengal. https://nomadit.co.uk/conference/iuaes2013/paper -download/paper/11711 (2006).

5. Islam, M. Z. et al. Variability assessment of aromatic rice germplasm by pheno-genomic traits and population structure analysis. Sci. Rep. 8, 9911. https://doi.org/10.1038/s41598-018-28001-z (2018).

6. Shiva, V. The violence of the green revolution: Third world agriculture, ecology, and politics. https://www.jstor.org/stable/j.ctt19 dzdcp (1991).

7. Dwivedi, J. L. Conserving genetic resources and using diversity in a flood-prone ecosystem in eastern India. http://www.idrc.ca/ books/focus/833/dwivedi.html (1997).

8. Deb, D. Seeds of Tradition, Seeds of Future: Folk Rice Varieties from East India (Research Foundation for Science Technology and Ecology, New Delhi, 2005).

9. Morishima, H. \& Oka, H. I. Genetic erosion in wild and cultivated species. RGN 12, 168-171 (1995).

10. Jackson, M. Preservation of rice strains. Nature 371, 470. https://doi.org/10.1038/371470a0 (1994).

11. Thrupp, L. A. Linking agricultural biodiversity and food security: The valuable role of agrobiodiversity for sustainable agriculture. Int. Aff. 76, 265-281 (2000).

12. Gliessman, S. R. Agroecology: The Ecology of Sustainable Food Systems 2nd edn. (Taylor and Francis, London, 2007).

13. Chatterjee, S. D. et al. The Rice Bio-diversity in West Bengal (Govt. of West Bengal, Department of Agriculture, Calcutta, 2008).

14. Hawkes, J. G. The Diversity of Crop Plants (Harvard University Press, Cambridge, 1983). 
15. Yadav, M. K. et al. Blast resistance in Indian rice landraces: Genetic dissection by gene specific markers. PLoS ONE 14(3), e0213566 (2019).

16. Ingole, K. D., Prashanthi, S. K. \& Krishnaraj, P. U. Mining for major blast resistant gens in rice landraces of Karnataka. Indian J. Genet. 74(3), 378-383 (2014).

17. Asibi, A. E. \& Coulter, J. A. Rice Blast: A disease with implications for global food security. Agronomy 9(8), 451 (2019).

18. Wang, G. L. et al. RFLP mapping of genes conferring complete and partial resistance to blast in a durably resistant rice cultivar. Genetics 136, 1421-1434 (1994).

19. Umakanth, B. et al. Diverse rice landraces of North-East India enables the identification of novel genetic resources for Magnaportheresistance. Front. Plant Sci. https://doi.org/10.3389/fpls.2017.01500 (2017).

20. Standardization evaluation system for rice. International Rice Research Institute, Philippines 5 (18) (2013).

21. Singh, V. K. et al. Marker assisted simultaneous but stepwise backcross breeding for pyramiding blast resistance genes Piz5 and Pi54 into an elite basmati rice restorer line 'PRR78'. Plant Breed. 132, 486-495 (2013).

22. Sadasivam, S. \& Manickam, A. Biochemical Methods for Agricultural Sciences (New Age International (P) Ltd., 1996).

23. Bradford, M. M. A rapid and sensitive method for the quantitation of microgram quantities of protein utilizing the principle of protein-dye binding. Anal. Biochem. 72, 248-254 (1976).

24. Kumar, A. et al. Resistant starch could be decisive in determining the glycemic index of rice cultivars. J. Cereal Sci. 79, 348-353 (2018).

25. Pal, K. et al. Analysis of rhizome colour content, bioactive compound profiling and ex-situ conservation of turmeric genotypes (Curcumalonga L.) from sub-Himalayan Terai region of India. Indus Crops Prod. 150, 112401. https://doi.org/10.1016/j.indcr op.2020.112401 (2020).

26. Hien, N. et al. Evaluation of aroma in rice (Oryzasativa L.) using KOH method, molecular markers and measurement of 2-acetyl1-pyrroline concentration. J. Trop. Agric. 50(4), 190-198 (2006).

27. Aljumaili, S. J. et al. Genetic diversity of aromatic rice germplasm revealed by SSR markers. Biomed. Res. Int. https://doi. org/10.1155/2018/7658032 (2018).

28. Kumar, R. et al. Analysis of genetic diversity in north Indian rice (Oryza sativa L.) germplasm, using SSR and ISSR markers. Rom. Agric. Res. 33 (2016).

29. Amiryousefi, A., Hyvonen, J. \& Poczai, P. iMEC: Online marker efficiency calculator. Appl. Plant Sci. 6(6) (2018).

30. Rohlf, F. J. NTSYS PC Numerical Taxonomy and Multivariate Analysis System, ver. 1.80 (Exeter Software, Setauket, 1995).

31. R: A language and environment for statistical computing. R Foundation for Statistical Computing, Vienna, Austria. https://www.Rproject.org/ (2020).

32. IBM Corp. IBM SPSS Statistics for Windows, Version 20.0 (IBM Corp., Armonk, 2011) https://www.ibm.com/analytics/spss-stati stics-software.

33. SAS Software, Copyright (c) by SAS Institute Inc., Cary, NC. https://www.sas.com/en_in/home.html (2002-2012).

34. Roy, B., Kumar, V., Tulsiram, S. D. \& Das, B. K. Development of high yielding aromatic mutants of rice (Oryzasativa L.) from a local aromatic cultivar, Tulaipanji by using gamma radiation. Indian J. Genet. 78, 409-416 (2018).

35. Chattopadhyay, T. et al. Biochemical and SSR marker based characterization of some Bengal landraces of rice suffixed with 'sail' in their name. Indian J. Genet. 68(1), 15-20 (2008).

36. Joshi, S. P., Gupta, V. S., Aggarwal, R. K., Ranjekar, P. K. \& Brar, D. S. Genetic diversity and phylogenetic relationship as revealed by inter-simple sequence repeat (ISSR) polymorphism in the genus Oryza. Theor. Appl. Genet. 100, 1311-1320 (2002).

37. Reddy, C. S., Babu, A. P., Swamy, B. P. M., Kaladhar, K. \& Sarla, N. ISSR markers based on GA and AG repeats reveal genetic relationship among rice varieties tolerant to drought, flood or salinity. J. Zhejiang Univ. Sci. B 10(2), 133-141 (2009).

38. Turki, T. A. A. \& Basahi, M. A. Assessment of ISSR based molecular genetic diversity of Hassawi rice in Saudi Arabia. Saudi J. Biol. Sci. 22, 591-599 (2015).

39. Chandel, G. et al. In vitro identification of low glycemic index (GI) white rice using Nutriscan GI Analyzer. ALS 5(23) (2016).

40. Zhou, Z., Robards, K., Helliwell, S. \& Blanchard, C. Composition and functional properties of rice. Int. J. Food Sci. Technol. 37(8), 849-886 (2002).

41. Jane, J. Y. et al. Effects of amylopectin branch chain length and amylose content on the gelatinization and pasting properties of starch. Cereal Chem. 76(5), 629-637 (1999).

42. Basu, S., Roychoudhury, A., Sanyal, S. \& Sengupta, D. N. Carbohydrate content and antioxidative potential of the seed of three edible indica rice (Oryzasativa L.) cultivars. Indian J. Biochem. Biophys. 49, 115-123 (2012).

43. Atkinson, F., Foster-Powell, K. \& Brand-Miller, J. C. International Tables of Glycemic Index and Glycemic Load Values: 2008. Diabetes Care 31(12), 2281-2283 (2008).

44. Deepa, G., Singh, V. \& Naidu, K. A. A comparative study on starch digestibility, glycemic and resistant starch of pigmented ('Njavara' and Jyothi) and non-pigmented ('JR 64') rice varieties. J. Food Sci. Technol. 47(6), 644-649. https://doi.org/10.1007/s1319 7-010-0106-1 (2010).

45. Yao, Y., Zhang, J. \& Ding, X. Structure-retrogradation relationship of rice starch in purified starches and cooked rice grains: A statistical investigation. J. Agric. Food Chem. 50, 7420-7425 (2002).

46. Kale, S. J. et al. Soaking induced changes in chemical composition, glycemic index and starch characteristics of basmati. Rice Sci. 22(4), 227-236 (2015).

47. Kale, S. J., Jha, S. K. \& Nath, P. Effects of variable steaming on chemical composition, starch characteristics, and glycemic index of basmati (Pusa Basmati 1121) rice. J. Food Process Eng. 40(6), e12567 (2017).

48. Asaduzzaman, M. et al. Comparisons of physiochemical, total phenol, flavanoid content and functional properties in six cultivars of aromatic rice in Bangladesh. Afr. J. Food Sci. 7(8), 198-203 (2013).

49. Verma, D. K. \& Srivastav, P. P. Proximate composition, mineral content and fatty acids analyses of aromatic and non-aromatic Indian rice. Rice Sci. 24(1), 21-31 (2017).

50. Katsantonis, D., Kadoglidou, K., Dramalis, C. \& Puigdollers, P. Rice blast forecasting models and their practical value: A review. Phytopathol. Mediterr. 56(2), 187-216 (2017).

51. Dean, R. et al. The top 10 fungal pathogens in molecular plant pathology. Mol. Plant Pathol. 13, 414-430 (2012).

52. Devi, S. \& Sharma, G. D. Blast disease of rice caused by Magnaporthegrisea: A review. Assam Univ. J. Sci. Technol. 6(1), 144-154 (2010).

53. Sharma, T. R. et al. Rice blast management through host-plant resistance: Retrospect and prospects. Agric. Res. 1(1), 37-52 (2012).

54. Kim, C. K. \& Choong, H. K. The rice leaf blast simulation model EPIBLAST. in Systems Approaches for Agricultural Development, 309-321 (Springer, New York, 1993).

55. Torres, C. Q. \& Teng, P. S. Path coefficient and regression analysis of effects of leaf and panicle blast on tropical rice yield. J. Crop Prot. 12, 296-302 (1993).

56. Mohanty, C. R., Gangopadhyay, S. \& Sahoo, K. Correlation and path-coefficient analysis study between some leaf characters and blast disease incidence at seedling stage of rice (Pyriculariaoryzae, Oryzasativa). Indian Phytopathol. 36, 508-515 (1983).

57. Jeyanandarajah, P. \& Seneviratne, S. N. S. Fungi seed-borne in rice (Oryza sativa) in Sri Lanka. Seed Sci. Technol. 19, 561-569 (1991).

58. Ou, S. H. Rice Diseases 2nd edn. (Commonwealth Mycological Institute, Kew, 1985). 
59. Liang, X. X. et al. Arabidopsis heterotrimeric G proteins regulate immunity by directly coupling to the FLS2 receptor. eLife $\mathbf{5}$, e13568 (2016).

60. Lin, F. et al. The blast resistance gene Pi37 encodes a nucleotide binding site leucine-rich repeat protein and is a member of a resistance gene cluster on rice chromosome 1. Genetics 177(3), 1871-1880 (2007).

61. Qu, S. H. et al. The broad-spectrum blast resistance gene Pi9 encodes a nucleotide-binding site-leucine-rich repeat protein and is a member of a multigene family in rice. Genetics 172(3), 1901-1914 (2006).

62. Zhai, C. et al. The isolation and characterization of $P i k$, a rice blast resistance gene which emerged after rice domestication. New Phytol. 189(1), 321-334 (2011).

63. Chen, X. W. et al. A B-lectin receptor kinase gene conferring rice blast resistance. Plant J. 46(5), 794-804 (2006).

64. Jin, C. P. et al. Identification of rice varieties resistant to rice blast in Jilin Province. Agta. Agric. Boreali-Sin. 26(3), 214-218 (2011).

65. Sun, G. Distirbutinon of resistance genes in rice and avirulence genes in rice blast fungus and molecule detection of Magnaporthe oryzae (University of Jinlin, Jinlin) (in Chinese with English abstract) (2012).

66. Hayashi, K., Hashimoto, N., Daigen, M. \& Ashikawa, I. Development of PCR-based SNP markers for rice blast resistance genes at the Pi-z locus. Theor. Appl. Genet. 108(7), 1212-1220 (2004).

67. Hayashi, K., Yoshida, H. \& Ashikawa, I. Development of PCR-based allele specific and InDel marker sets for nine rice blast resistance genes. Theor. Appl. Genet. 113(2), 251-260 (2006).

68. Sharma, T. R. et al. High-resolution mapping, cloning and molecular characterization of the gene of rice, which confers resistance to rice blast. Mol. Genet. Genom. 274(6), 569-578 (2005).

69. Jia, Y., Wang, Z. \& Singh, P. Development of dominant rice blast Pi-ta resistance gene markers. Crop Sci. 42(6), 2145-2149 (2002).

70. Ingole, K. D., Prashanthi, S. K. \& Krishnaraj, P. U. Mining for major blast resistance genes in rice landraces of Karnataka. Indian J. Genet. Plant Breed. 74(3), 378-383 (2014).

71. Tian, H. G. et al. Effect of introgressed Pigm gene on rice blast resistance and yield traits of japonica rice in cold area. J. Shenyang Agric. Univ. 47(5), 520-526 (2016).

72. Yan, L. et al. Molecular screening of blast resistance genes in rice germplasms resistant to Magnaporthe oryzae. Rice Sci. 24(1), 41-47 (2017).

73. Fuentes, J. L. et al. Identification of microsatellite markers linked to the blast resistance gene Pi-1 (t) in rice. Euphytica 160, 295-304 (2008).

74. Ashikawa, I. N. et al. Two adjacent nucleotide-binding site-leucine-rich repeat class genes are required to confer $P i k m$-specific rice blast resistance. Genetics 180, 2267-2276 (2008).

75. McCouch, S. R. et al. Development and mapping of 2240 new SSR markers for rice (Oryzasativa L.). DNA Res. 9, 199-207 (2002).

76. Zhu, M., Wang, L. \& Pan, Q. Identification and characterization of a new blast resistance gene located on rice chromosome 1 through linkage and differential analyses. Phytopathology 94, 515-519 (2004).

77. Berruyer, R. et al. Identification and fine mapping of Pi33, the rice resistance gene corresponding to the Magnaporthe grisea avirulence gene ACE1. Theor. Appl. Genet. 107, 1139-1147 (2003).

\section{Acknowledgements}

The authors are grateful for financial support under the project "Development of blast resistance high- yielding short grain aromatic rice variety for northern Bengal" funded by ICAR, Govt. of India under Niche area of Excellence (NAE) scheme with project reference number Edn.5(12)/2017-EP\&HS. The authors would also like to acknowledge Dr. Rupsanatan Mondal, UBKV for critical input during the analysis of molecular marker data. The authors would also like to acknowledge, the Nodal officer, GKMS, for providing us the meteorological data during the study period.

\section{Author contributions}

D.M.-Conduction of the experiments and collection of data. P.R.-Conduction of the experiments. S.J.Conduction of the experiments. G.S.S.-Conduction of the experiments. A.B.-Statistical analysis of the data. H.C.-Analysis of the marker data. S.M.-Execution of biochemical experiments, writing of the manuscript, management of fund. N.S.-Overall planning of the research program and execution of field and laboratory experiments, writing of manuscript, management of fund. B.R.-Collection and maintenance of genotypes, writing of manuscript, management of fund. P.M.B.-Isolation and maintenance of pathogen, management of fund. A.K.C.-Writing of manuscript, management of fund. A.C.-Administrative managements, critical input. All authors reviewed the manuscript.

\section{Competing interests}

The authors declare no competing interests.

\section{Additional information}

Supplementary Information The online version contains supplementary material available at https://doi. org/10.1038/s41598-021-83921-7.

Correspondence and requests for materials should be addressed to N.S.

Reprints and permissions information is available at www.nature.com/reprints.

Publisher's note Springer Nature remains neutral with regard to jurisdictional claims in published maps and institutional affiliations. 
(c) (i) Open Access This article is licensed under a Creative Commons Attribution 4.0 International cc) License, which permits use, sharing, adaptation, distribution and reproduction in any medium or format, as long as you give appropriate credit to the original author(s) and the source, provide a link to the Creative Commons licence, and indicate if changes were made. The images or other third party material in this article are included in the article's Creative Commons licence, unless indicated otherwise in a credit line to the material. If material is not included in the article's Creative Commons licence and your intended use is not permitted by statutory regulation or exceeds the permitted use, you will need to obtain permission directly from the copyright holder. To view a copy of this licence, visit http://creativecommons.org/licenses/by/4.0/.

(C) The Author(s) 2021 